\title{
Inducible Control of Tissue-specific Gene Expression in Xenopus tropicalis
}

\author{
Jeiwook Chae \\ Seoul, Korea \\ B.S., Sungkyunkwan University, 1996 \\ M.S., Kwangju Institute of Science and Technology, 1998
}

A dissertation presented to the Graduate Faculty of the University of Virginia in Candidacy for the Degree of Doctor of Philosophy

Department of Biology

University of Virginia

January 2005

Robert M. Grainger, Ph.D.

(Dissertation Advisor)

Paul N. Adler, Ph.D.

(First Reader)

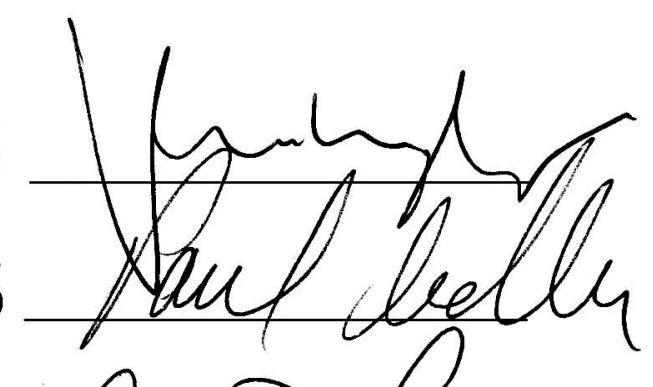

Carla B. Green, Ph.D.

.

Jay Hirsh, Ph.D.

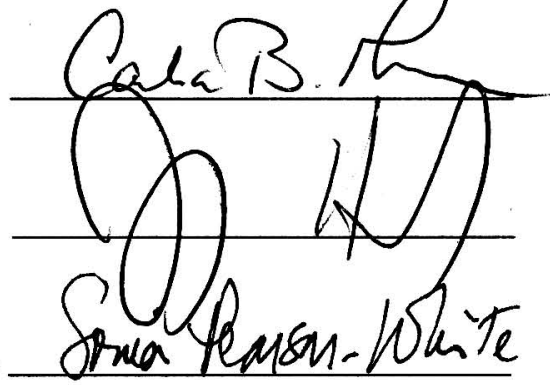

Sonia H. Pearson-White, Ph.D. 
(C) Copyright by

Jeiwook Chae

All rights reserved

January 2005 


\begin{abstract}
Vertebrate eye formation has been extensively studied in amphibian embryos as a model for embryonic induction. More recently, a number of putative regulatory genes involved in eye determination have been identified. However, their defined functions during eye formation remain to be examined. Combining recent technological breakthroughs, e.g. a procedure for frog transgenesis and a new genetic system, Xenopus tropicalis, I have performed a series of experiments to test the function of these genes in eye determination.

The primary goal of the dissertation is to define the function of an essential regulatory gene, Otx2, controlling eye determination. To accomplish this, first, the transgenesis technique was used to express engineered mutations of this gene in a tissuespecific manner, resulting in ocular defects whose analysis was complicated by the background abnormalities associated with this nuclear transfer technique. To overcome these difficulties, the feasibility of using a hormone-inducible GAL4/UAS system permitting establishment of stable lines and consistent spatiotemporal control of transgene expression was tested in X. tropicalis. The expression of a UAS-target reporter gene was efficiently activated in a tissue-specific manner by a ligand-inducible GAL4 in response to RU486.
\end{abstract}

This hormone-inducible system was then utilized to functionally inactivate Otx2 in the eye through the induced expression of a dominant negative construct as an example of tissue-specific gene repression approach. Embryos inheriting both the GAL4 activator and dominant negative effector displayed consistent profound eye defects in an RU486- 
dependent fashion. Early morphogenetic movements of the optic vesicle fail, which normally position the dorsal presumptive retinal pigmented epithelium (RPE) in juxtaposition to the distal neural retina, separating the double-layered optic cup from the neural tube. As a result, the RPE cells remain dorsally, the choroid fissure fails to close, and the eyes remain fused to the forebrain vesicle. These results indicate that $O t x 2$ plays a critical role in ocular development, in particular, in controlling the morphogenesis of the transition from the optic vesicle to optic cup.

In addition to helping to clarify the role of Otx 2 during eye development, these studies provide the groundwork for future experiments utilizing the inducible GAL4/UAS system to study other gene functions in the frog. 


\section{Table of Contents}

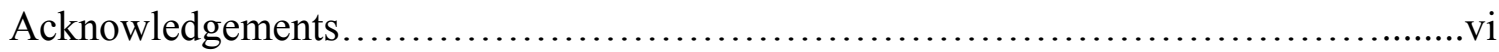

Chapter 1. Introduction to Xenopus tropicalis and a ligand-inducible gene control

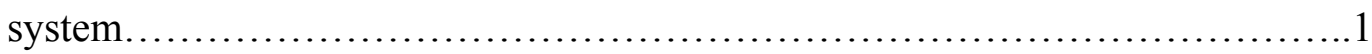

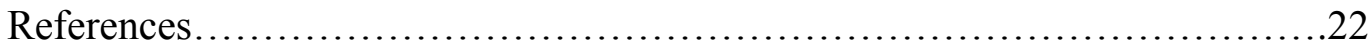

Chapter 2. Inducible control of tissue-specific transgene expression in X. tropicalis lines: development of hormone-regulatable GAL4/UAS system in Xenopus.................35

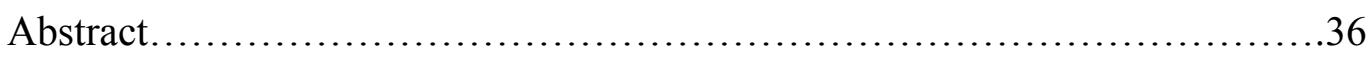

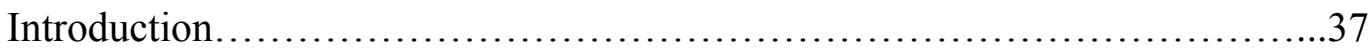

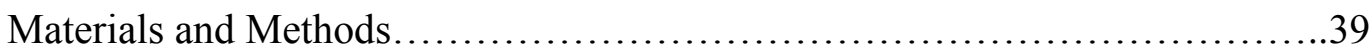

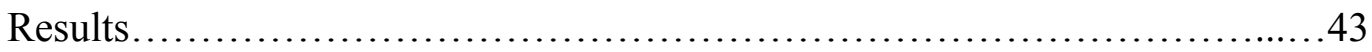

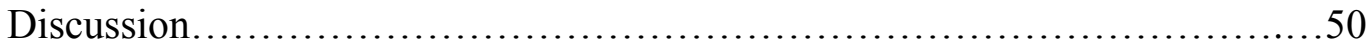

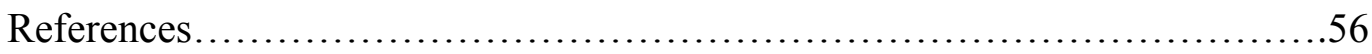

Chapter 3. Functional inactivation of Otx2 in the eye: application of inducible GAL4 system as tissue-specific gene repression approach.............................................74

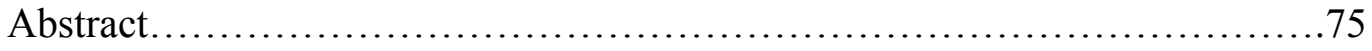

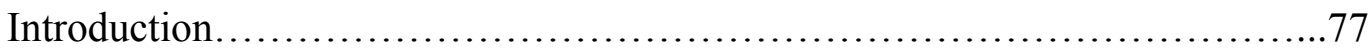

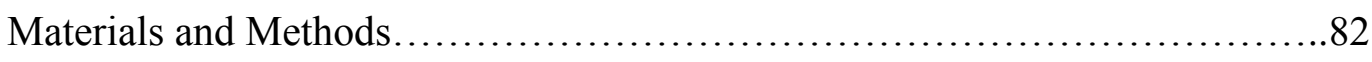

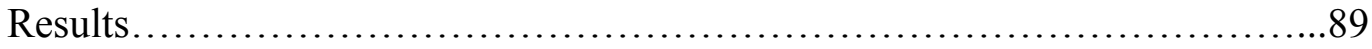

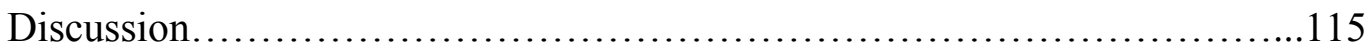

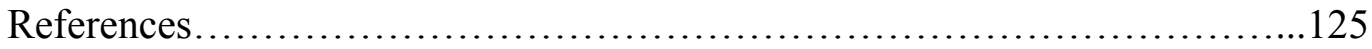

Chapter 4. Discussion and future applications...........................................................162

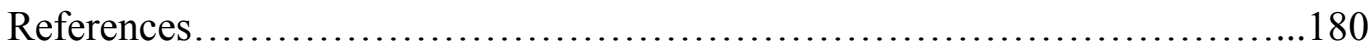




\section{Acknowledgements}

I would like to thank my advisor, Dr. Robert Grainger for his support and patience, and for educating me in many aspects of science over the last years. His perfectionism and enthusiasm for doing biology will affect me as a scientist throughout my career.

I also would like to thank all the members of my committee for their support and advice. Especially my first reader, Paul Adler who has been very generous with his time and thoughtful advice.

I am also very grateful to all the Grainger lab members (past and present) for their support and friendship. My special thanks to Marilyn Fisher: My thesis would not have been the same without your help, Marilyn.

I'd like to thank my family from the bottom of my heart. Mom, Jangin Jangmo nim, Limi, KC, Leo, and Simon. They have been nothing but supportive for everything I have done. I would never have made it without your help and support I received all along the way. I also want to give my special thanks to my lovely children, Hannah and David, who have been, whether they realize it or not, encouraging me in what I am doing.

Finally, I want to thank my better half, Kyungsun for her endless support, patience and love, and for always being there for me throughout the ups and downs of last six years of my Ph.D. work. 


\section{Chapter 1:}

Introduction to Xenopus tropicalis and a ligand-inducible gene control system 


\section{1-1. Eye determination: a model for vertebrate organogenesis}

The problem of how cells become determined to form complex tissues and organs is a fundamental one in developmental biology. Presently we understand that a series of inductive events during early vertebrate development is responsible for such complicated developmental processes as the formation of particular germ layers and tissues. The first observations and experiments elucidating the concept of embryonic induction, i. e. how one tissue depends on another for its formation, came from the analysis of a relatively simple biological process, the formation of lens in the eye. Embryonic eye development is, by itself, an important problem because of a general interest in understanding how the visual process is generated during embryonic development. It also has been one of the most intensively studied examples of vertebrate cell determination (reviewed by Grainger, 1996) because of the relative simplicity of the developing lens and its interaction with presumptive retina, the optic vesicle. Optic vesicles are evaginated from the newly formed neural tube bilaterally and subsequently make contact with presumptive lens ectoderm. In the classic view of lens induction, lens determination seemed dependent upon the location and inductive influence of the developing retina that was thought to be both necessary and sufficient for lens formation. This original suggestion that only the optic vesicle can induce the lens has been the basis for the stated principle that inductions may involve only one tissue interaction. However, subsequent studies of this phenomenon have suggested that this view is oversimplified, and lens induction is far more complex (reviewed by Saha et al., 1989) than a simple one-step model suggested by the classical view. It requires a number of inductive events (Henry 
and Grainger, 1987; Henry and Grainger, 1990) that include activation of a series of eye regulatory genes (Zygar et al., 1998). Although a number of recent studies have made a great deal of progress in identifying many regulatory genes that seem to be involved during vertebrate eye development (Hirsch and Grainger, 2000; Viczian et al., 2003), how their functions are integrated, spatially and temporally, at the molecular level during lens and retinal specification remains to be revealed. Among these genes, Otx2 and Pax6 are emphasized on this thesis, because Pax6 is believed to be a master controller in eye determination and the expression patterns of Otx2 indicate its possible roles in both lens and retinal development.

\section{1-2. Homeobox genes involved in eye development}

Recent studies on a number of regulatory genes involved in eye development have enabled researchers to begin to dissect the regulatory processes of eye determination at the molecular level. Among the many genes that are believed to be important in eye development, Pax6 and Otx2 are of special interest for this dissertation.

Pax6 encodes a transcription factor that contains both paired box and homeobox DNA binding domains and functions in the development of eyes and central nervous system (reviewed by Ashery-Padan and Gruss, 2001; Callaerts et al., 1997). Pax6 is expressed early in development in the forebrain, hindbrain, and spinal cord as well as the lens ectoderm and developing retina in Xenopus and mouse (Hirsch and Harris, 1997; Walther and Gruss, 1991). Not only the amino acid sequences, but also the genome structure and expression patterns of Pax6 have been found to be highly conserved 
throughout organisms as diverse as Caenorhabditis elegans, Drosophila, mouse, and human (Chisholm and Horvitz, 1995; Quiring et al., 1994; Ton et al., 1991; Zhang and Emmons, 1995). In addition, targeted expression of either eyeless, the Drosophila homolog of this gene or the mouse Pax6 gene induces ectopic eye formation in Drosophila. Although these results argue for Pax6 as a master controller in eye development (Chow et al., 1999; Halder et al., 1995), there are other transcriptional regulators including the Six family genes which are believed to play a crucial role in ocular development (Hirsch and Grainger, 2000; Ogino and Yasuda, 2000; Pichaud and Desplan, 2002).

Heterozygous and homozygous mutations in Pax6 result in genetic disorders in the development of both murine and human sensory systems, such as Aniridia in man (Jordan et al., 1992) and Small eye (Sey) in mice (Hill et al., 1991). Thus, eye development seems to be remarkably susceptible to a reduction of Pax6 gene expression. Surprisingly, mice carrying multiple copies of the human Pax6 gene under the control of its complete regulatory regions on a wild-type background also show specific developmental abnormalities of the eye (Schedl et al., 1996). Therefore, not only reduced, but also increased Pax6 gene dosage causes developmental defects in the eye, suggesting an exceptional sensitivity of cells within the eye to changes in levels of this transcriptional regulator. However, abnormalities in eye formation in Sey or Pax6overexpressing mice could be due to the role of Pax6 in the lens itself or due to its effects in retinal development since this gene is expressed in both tissues. The test of this hypothesis in Xenopus by transgenic assays using tissue-specific promoters will allow us to investigate the fundamental role of Pax6 in vertebrate eye development, which was not 
possible previously by conventional mRNA injection methods. During the course of this study, the questions of cell-autonomous function of Pax6 in lens and retinal development have been addressed in mouse model using gene targeting technique (Ashery-Padan et al., 2000a; Marquardt et al., 2001). Although mice are uniquely valuable for their homologous recombination-based reverse genetics, they suffer from several limitations including high cost of gene targeting experiments and small inaccessible embryos, which make some embryological assays extremely challenging. In contrast, X. tropicalis embryos are very suitable for standard embryological manipulations. This allows further embryological assays to elucidate the role of genes (e.g. $\operatorname{Pax} 6$ ) after gene inactivation or overexpression experiments using powerful frog transgenic system (Hartley et al., 2001; Hirsch et al., 2002b; Kroll and Amaya, 1996) that allows the generation of hundreds of transgenic embryos with relatively very low cost.

Otx2 is one of the homologs of orthodenticle, a Drosophila gene implicated in the control of head development (Finkelstein et al., 1990). This gene encodes a bicoid class homeodomain containing transcription factor (Simeone et al., 1993). The mouse Otx2 is first expressed in the entire epiblast of pre-streak stage embryos and later in anterior neuroectoderm, corresponding to presumptive forebrain and midbrain (Mallamaci et al., 1996; Simeone et al., 1993). In Xenopus, during early gastrulation stages, this gene is expressed in anterior mesendoderm tissue and later restricted to neural and anterior head ectoderm. From neurula stages onward its expression domains include the optic vesicle and nasal placode as well as lens placode (Blitz and Cho, 1995; Pannese et al., 1995; Zygar et al., 1998). In targeted mice Otx2 mutants (Acampora et al., 1995; Ang et al., 1996), the entire head structure including the eye is missing. Therefore, it is clearly 
uninterpretable whether there is a tissue-autonomous effect of the mutation in Otx2 on ocular development, although its expression in both developing retina and lens implicates the role of this gene in the eye development.

Recently, evidence has accumulated from several sources, however, supporting the idea that $O t x$ genes may be directly involved in vertebrate eye formation (MartinezMorales et al., 2001; Nishida et al., 2003; Viczian et al., 2003). This will be further discussed in detail in Chapter 3. Briefly, the ocular phenotype of mice carrying different functional copies of $O t x 1$ and $O t x 2$ has been analyzed, and it has been shown that these genes are required in a dose-dependent manner for the normal development of the eye (Martinez-Morales et al., 2001). Results from lipofection studies of Xenopus retinal precursors with either wild-type Otx2 or its dominant negative form suggested that this gene product promotes bipolar cell fates (Viczian et al., 2003). However, the abnormalities in retinal formation in Otx mutant mice could be directly due to decreased levels of Otx2 activity in the retina itself, or indirectly due to low levels of OTX2 protein in the lens ectoderm as similar abnormal retinal phenotype has indeed been observed in a lens-specific Pax6 conditional mouse mutation (Ashery-Padan et al., 2000b). To test this hypothesis properly, the gene activity should be inactivated specifically in the retina or lens ectoderm. To accomplish this, it is of particular importance to target expression of dominant inhibitory gene construct to particular tissues, lens or retina. Recent technical advances in Xenopus now allow the delivery of dominant negative constructs to eye tissues using tissue-specific promoter in transgenic assays. 


\section{1-3. Xenopus tropicalis, a new vertebrate genetic system}

Significant embryological innovations of methods in the frog system have been extremely important for studies on many early developmental phenomena. By combining useful perspectives from Xenopus with the power of genetics, Xenopus tropicalis (reviewed by Amaya et al., 1998; Hirsch et al., 2002a; Khokha et al., 2002; Vogel, 1999), a close relative of Xenopus laevis, would offer more profound understanding of complex developmental processes such as vertebrate eye development. Xenopus laevis has been one of the most favored model systems for vertebrate experimental embryology. One of main reasons for this is that large size and robustness of Xenopus embryos and their external development allow scientists to readily perform embryological manipulations; the embryos are extremely tolerant to transplantation and tissues are hardy enough to differentiate in an explant (Moore et al., 1995; Wilson et al., 1989). However, X. laevis has a major drawback for the purpose of molecular genetic studies; the species is tetraploid and therefore has four copies of most of genes instead of the usual two. The extra copies make it virtually impossible to do genetic studies since it may be necessary to inactivate all four copies of a gene to create mutants. The presence of inactive pseudogenes and loss of regulatory elements in different copies of a given gene also greatly complicates isolation and analysis of promoter sequences.

Xenopus (Silurana) tropicalis is the only close relative of $X$. laevis that has a diploid genome (Amaya et al., 1998; Hirsch et al., 2002a). X. tropicalis' diploid genome and its several other features such as a shorter generation time (3-5 months instead of 1-2 years of $X$. laevis) and a smaller adult size permits large-scale multigeneration genetic 
analyses. Recent development of a method for generating transgenic frogs (Hirsch et al., 2002b; Kroll and Amaya, 1996) in a large scale also contributes to make X. tropicalis a vertebrate genetic model system. While $X$. tropicalis' eggs are slightly smaller than $X$. laevis, $X$. tropicalis shares virtually all of $X$. laevis advantages as an embryological system. The two species are close enough that key stages in development follow morphological features of $X$. laevis, although $X$. tropicalis develops faster (Khoka et al., 2002).

The transgenic procedure for integrating genes (Kroll and Amaya, 1996) has revolutionized the possibilities for examining gene expression that was not previously possible in the Xenopus system. This new technique has made it possible to test the expression of promoters and tissue-specific enhancer sequences that have recently been isolated from Xenopus in living animals. The procedure involves the following steps. Briefly, linearized plasmid DNA is introduced into isolated decondensed sperm nuclei in vitro and then single nuclei are transplanted into unfertilized eggs, producing normal diploid transgenic embryos that develop to advanced stages. Integration of DNA into the chromosome of the nuclei is very efficient using this procedure, such that more than $50 \%$ of the resulting embryos are transgenic. Transgenic embryos have shown the reporter gene expression driven by a number of promoters with high tissue specificity, thus reflecting their endogenous expression patterns. Another great advantage of this method over previous transgenesis in mice and zebrafish is that the resulting transgenic embryos express the integrated genes nonmosaically because the transgene is integrated into the male genome of sperm nuclei prior to fertilization. Therefore, further breeding of animals is not required to obtain nonmosaic expression. It also has been confirmed that the 
germline transmission of transgenes introduced by this technique occurs in the expected Mendelian fashion (Bronchain et al., 1999; Hirsch et al., 2002a). This transgenesis method, originally developed for Xenopus laevis, has been modified for and shown to be effective in Xenopus tropicalis (Offield et al., 2000). Transgenic reporter constructs in $X$. tropicalis have been shown to reproduce the endogenous pattern of expression of several promoters cloned from $X$. laevis (Hirsch et al., 2002b).

The potential of $X$. tropicalis as a genetic model system (Hirsch et al., 2002a) has been evaluated in our lab by characterization of putative $X$. tropicalis mutants which were generated by conventional chemical mutagenesis, random insertional mutagenesis using gene-trap techniques (Bronchain et al., 1999) and gynogenesis (Krotoski et al., 1985; Noramly and Grainger, 2002). Presently we have over 30 reporter transgenic lines of $X$. tropicalis bearing Green or Red Fluorescent Protein (GFP or RFP) under the control of tissue-specific and ubiquitous regulatory elements (Hirsch et al., 2002b). Several of these lines have already reached the F2 or F3 generations with no alterations in transgene expression. One of these tissue-specific regulatory sequences, the retinaspecific $X$. tropicalis $R x$ gene (Mathers et al., 1997) promoter that contains its endogenous $R x$ expression pattern in GFP reporter lines was used to drive expression of the chimeric GAL4 transactivator in pRx-GALPR transgenic lines (see Chapter 2).

\section{1-4. Inducible systems controlling transgene expression}

One of disadvantages in Xenopus as compared with the mouse is the absence of a technique for altering the function of a known gene by targeted modification. In Xenopus, 
the most common way to analyze the function of any cloned gene is to misexpress its wild-type product, or the dominant-negative variant of it, by mRNA injection into blastomeres (e.g., Chow et al., 1999; Smith and Harland, 1991; Smith and Harland, 1992). Although this method rapidly provides insights into the developmental function of a gene, it is unspecific with regard to the tissue and developmental stage of expression. This obscures determining the spatiotemporal function of a gene product in a given process. For example, misexpression of a dominant negative form of Otx2 by mRNA injection into blastomeres has been shown to result in loss of anterior structures such as the eye in severely affected animals (Isaacs et al., 1999). However, the pleiotropic effect of Otx2 on both eye and brain make this eye defect difficult to interpret. Ubiquitous misexpression of dominant-negative activities also often raises the argument that they might lead to misleading results due to their interfering with other unrelated signaling pathways in addition to the target (Hemmati-Brivanlou and Melton, 1992; Herskowitz, 1987). In addition, very high levels of gene activity by overexpression could result in non-physiological effects.

The ability to express a gene product in a directed stage- and tissue-specific manner is a powerful way of analyzing its role in development. One of the common methods for manipulating gene expression is to drive expression of the target gene by fusing it downstream of a defined promoter (Parkhurst and Ish-Horowicz, 1991; Robinson et al., 1995; Zhang et al., 2003). This method, combined with a dominantnegative strategy, can be used to delete particular gene activity in a tissue-specific manner. The use of a tissue-specific promoter or one of regulatory cis-acting elements from a characterized promoter/enhancer allows transcription of dominant-inhibitory 
activities to be restricted in a defined subset of cells at a specific developmental stage. This approach permits direct testing of the function of a particular gene in the determination of a specific tissue, especially when the gene of interest is expressed, and it functions in more than two distinct tissues and/or at more than two different stages of development.

Altering transcription patterns of a gene also has been a powerful way of determining its function (Broccoli et al., 1999). This analysis is particularly useful for defining the roles of genes encoding putative transcriptional activators (or repressors) since these genes can be expressed in a different cell, or at a different time using tissuespecific promoters. Their effects on the subsequent expression of target genes and a switch in cell fate can be assayed. In this way it is possible to determine if the activator is both necessary and sufficient for transcription of the target and responsible for cell fate determination. Over- or misexpression phenotypes can also be extremely informative about gene function (Rorth, 1996; Rorth et al., 1998). For example, misexpression of homeotic genes in Drosophila causes a striking transformation of body segmentation (Lewis, 1978; Schneuwly et al., 1987). Ectopic expression of the eyeless gene results in the formation of ectopic eye tissues (Halder et al., 1995) and increased levels of Pax6 activity causes specific defects in the eye as shown in mice carrying multiple copies of the human Pax6 gene (Schedl et al., 1996). The fact that simple overexpression of a human gene in transgenic animals causes developmental defects suggests that analysis of overexpression phenotypes by targeted gene expression can be very useful to understand specific genetically inherited human diseases. Abnormalities of the eyes have been indeed reported in cases with a duplication in the chromosomal region encompassing 
PAX6 (Schedl et al., 1996 and references therein). In addition, it has been estimated that over two thirds of all Drosophila, C. elegans and yeast genes (and probably even more in vertebrates) show no obvious loss-of-function phenotypes (Dujon et al., 1994; Miklos and Rubin, 1996; Sulston et al., 1992), perhaps due to functional redundancy. For these genes, ectopic expression phenotypes can provide important information about their functions.

Until recently there has been no way to address the issues discussed above in Xenopus. However, the transgenic procedure for integrating genes that has been developed for Xenopus (see above), now permits the expression of a gene product in a tissue-specific manner using a defined promoter. Analysis of the consequences of

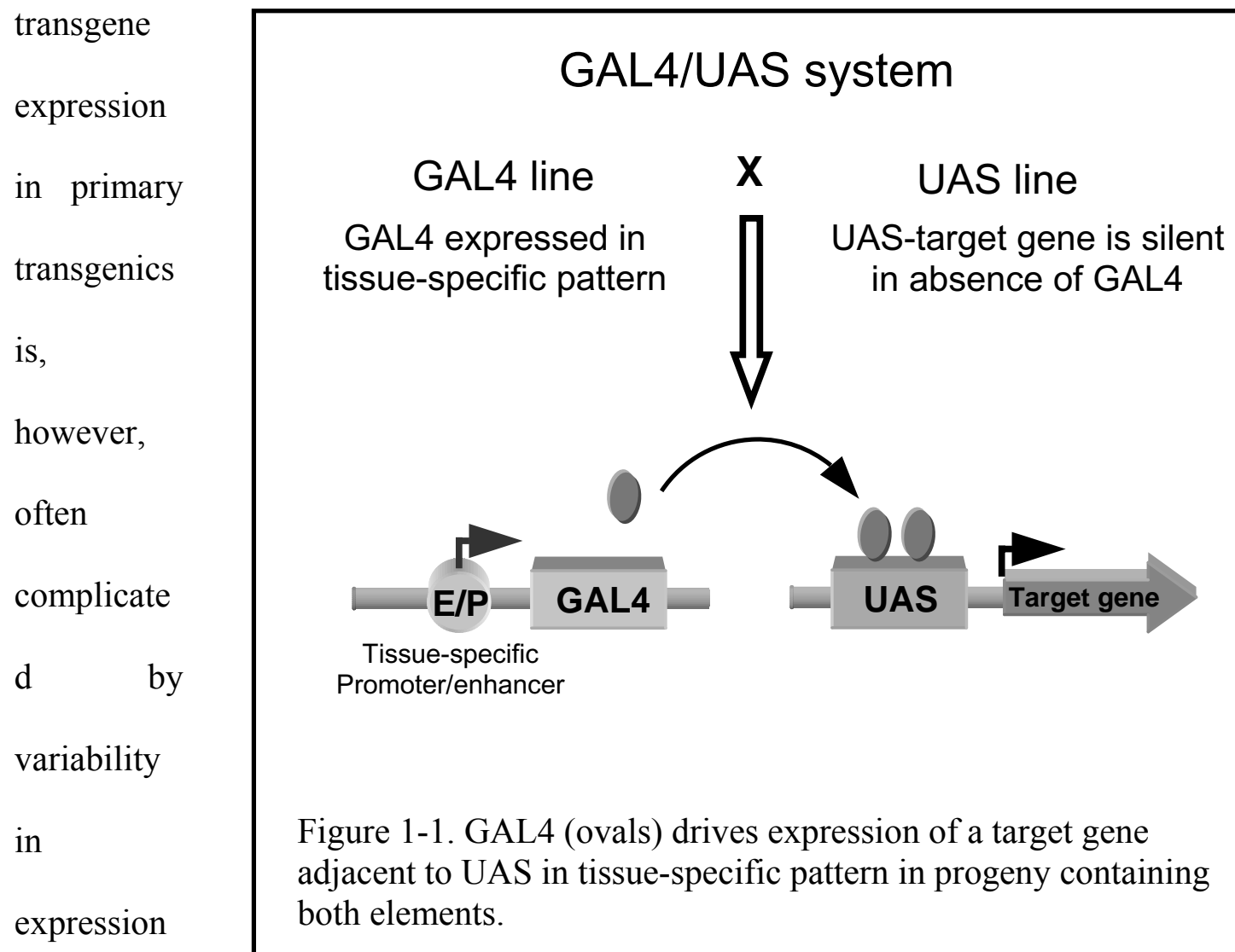

\section{GAL4/UAS system}

1-1. GAL4 (ovals) drives expression of a target gene adjacent to UAS in tissue-specific pattern in progeny containing both elements.

levels and background abnormalities associated with nuclear transfer technique. These 
problems can be circumvented by establishing stable transgenic lines, but only if transgene expression is non-lethal. The GAL4/UAS targeted expression system (Brand and Perrimon, 1993) can overcome these complexities. This binary system separates the target gene (UAS-effector) from its transcription activator (GAL4 transactivator) in two different transgenic lines (see Fig. 1-1). In the GAL4 lines, the yeast transcriptional activator GAL4 is present, but has no target gene to activate. In the UAS-target line, the target gene under the control of GAL4 binding site (Upstream Activation $\underline{\text { Sequences) }}$ remains silent in the absence of the activator. It is only when the two lines are crossed that the GAL4-responsive target gene is turned on in the progeny carrying both the effector and transactivator transgenes. This ensures that transgenic founders are viable even if the target gene product is toxic to the organism. Another advantage of this system over the primary transgenic approach is that the embryos expressing both the constructs are homogeneous: all resultant embryos will display the consistent phenotype, if there's any, resulting from the expression of exactly the same copy number of the transgenes. This method also removes developmental abnormalities associated with the primary transgenic procedure, allowing us to focus on the phenotype caused by the target gene expression. Despite the virtues of the GAL4/UAS system such as transgene expression in a spatially restricted manner, this method provides no control over the timing of GAL4 expression other than that intrinsic to the driver promoter. Because early dominant pleiotropic effects of mis- or overexpressed transgenes can obscure functional analysis at later developmental stages, temporal control over the tissue-specific expression of transgenes is often desirable. 
Temporal control of transgene induction has generally been obtained by using heat shock promoters in Drosophila (D'Avino and Thummel, 1999) and more recently in Xenopus (Wheeler et al., 2000). This promoter lacks spatial control, however; it is expressed ubiquitously.

To circumvent this problem, several other approaches that offer potential for regulating gene expression in both time and space have been proposed and evaluated. The next section will give a brief summary of different reagent-inducible regulatory systems that Ligand-inducible regulator E/P Transcription factor

Tissue-specific Enhancer/promoter
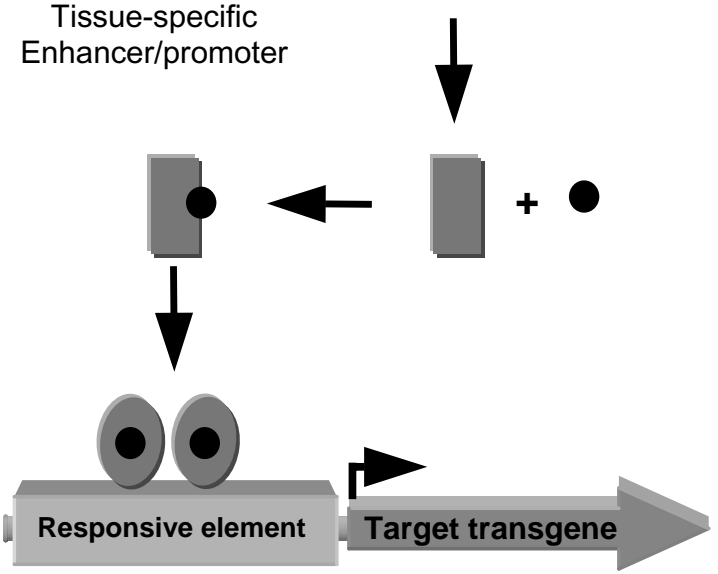

Figure 1-2. The ligand-dependent transcription factor (rectangle) is inactive without a ligand. Its binding to a ligand (circle) causes a conformational change (oval), allowing the regulator to bind to response element and to activate the target gene transcription.

have been developed in

transgenic models over the last few years and will then focus on a steroid hormone ligand binding domain based regulatory system that is utilized with the GAL4 activator to study Otx2 in this dissertation. In general, the ligand-inducible binary gene regulatory systems are based on transcription factors whose activity is regulated by a ligand (see Fig. 1-2). The ligand-dependent regulator (rectangle) is inactive until it binds to a ligand (circle) that causes a conformational change (oval), permitting its binding to its cognate 
responsive sequence and activate the target gene transcription. The desirable goals of these inducible systems are to achieve low background expression with a high level of rapid induction upon administration of a nontoxic easily deliverable ligand (Burcin et al., 1998; Burcin et al., 1999).

\section{IPTG based regulatory systems}

This inducible system is based on the bacterial lactose repressor protein (lacl) and its cognate DNA-binding sites, the short lac operator (lacO) sequences (Jacob and Monod, 1961), which are embedded upstream to the TATA box of a tissue-specific promoter linked to the coding sequence of a target transgene. In the absence of the inducer, lac repressor binds to the lac operators and prevents transcription. This repression can be derepressed only in the presence of isopropyl beta-D-thiogalactoside (IPTG) by causing the repressor to vacate the operaters, and the target gene expression is activated in a tissue-specific manner (Cronin et al., 2001; Deuschle et al., 1989; Gossen et al., 1993). In addition, there are mutant form of lacI repressors whose activity is regulated in a reverse manner to the wild-type repressor: repression is dependent on or increased by the inducer IPTG (Myers and Sadler, 1971). One of these mutant repressors was modified by replacing its repressor domain with the activation domain of a viral protein, creating a lacI activator protein (LAP; Baim et al., 1991). LAP can drive the expression of lacObearing transgenes in the presence of IPTG. These lac repressor systems have successfully achieved high induction of reporter target genes in transgenic mice (Cronin et al., 2001; Ryan and Scrable, 2004) as well as in mouse cell lines (Baim et al., 1991). 
However, there is a disadvantage of potential toxicity of IPTG, especially to mammalian cells (Burcin et al., 1998). The application of this system in frog remains to be tested.

\section{Tetracycline based regulatory systems}

The tetracycline (tet)-inducible system is based on binding of tetracycline operator (tetO) sequences, either by the tetracycline transactivator protein (tTA) in the absence, or by a mutant reverse transactivator (rTA) in the presence, of tetracycline (Bello et al., 1998; Bieschke et al., 1998; Gossen et al., 1994; Gossen et al., 1995; Stebbins and Yin, 2001). Upon the administration of the tetracycline analog, doxycycline, the tTA under the control of a tissue-specific enhancer drives the expression of a transgene flanked by tetO. The tTA system has been reported to function in Drosophila (Bello et al., 1998; Bieschke et al., 1998; Stebbins et al., 2001) and transgenic mice (Morozov et al., 2003; Shockett et al., 1995; Shockett and Schatz, 1996). However, a significant disadvantage using this regulatory system is that the continuous presence of high doses of antibiotics over a long time period, which is required to repress the target transgene, can have non-specific side effects on transgenic animals (Burcin et al., 1998; Mansuy et al., 1998). A relatively slow induction of the transgene expression also has been reported in studies using Drosophila. The expression was first detected $6 \mathrm{hr}$ after tetracycline withdrawal, with a day or more for full expression (Bello et al., 1998). These IPTG and tetracycline based regulatory systems are conceptually elegant but have the practical limitations of potential toxicity to the animals. 


\section{GAL4-hromone receptor fusion-based systems}

The generation of a GAL4-based inducible system can take advantage of the extensively used GAL4/UAS elements to target gene expression, arguably the most extensively characterized regulatory system. The GAL4/UAS system has been utilized in Drosophila extensively and more recently in other transgenic animal models including mice (Ornitz et al., 1991) and zebrafish (Scheer and Camnos-Ortega, 1999). There are two GAL4-based pharmacologically inducible systems that offer temporal control of transgene expression over spatial regulation by tissue specific promoters. The inducibility of these GAL4 proteins is accomplished by engineering the activators to generate GAL4nuclear steroid hormone receptor fusions whose transcriptional activity can be regulated by steroid hormones or chemically related analogues. Therefore, these modified forms of GAL4 proteins that are expressed by tissue-specific promoters but activated only in the presence of the exogenous compounds provide ligand-inducible transactivation of UASlinked target genes. One is based on the estrogen-receptor-GAL4 fusion protein (GAL4ER, Han et al., 2000). The other is based on the GAL4 fusion protein with the progesterone ligand-binding domain (GL-VP or GALPR, Burcin et al., 1998; Chae et al., 2002; Wang et al., 1994). Another ligand, steroid hormone receptor-based regulatory system utilizes fusions between the ligand-binding domain of the glucocorticoid receptor (GR) and a heterologous protein. Although efficient hormone-inducible gene expression has been reported in transfected mammalian tissue culture cells (Hollenberg et al., 1993) and early Xenopus embryos injected with an mRNA of the GR ligand-binding domain fusion (Kolm and Sive, 1995), the applicability of this method in transgenic animals has yet to be tested. 


\section{Estrogen-receptor-GAL4 fusion based system}

This estrogen-inducible regulatory system utilizes a chimeric regulator GAL4-ER, consisting of the ligand-binding domain of the estrogen receptor (ER) fused to the GAL4 DNA binding domain and a herpes virus VP16 transcriptional activation domain (Braselmann et al., 1993; Burcin et al., 1998). In the presence of estrogen the chimeric regulator dimerizes, binds to the GAL4-responsive UAS sequence and activates target gene transcription. The utilization of GAL4-ER for temporally regulated gene expression has been reported in mammalian cells (Braselmann et al., 1993) and in transgenic flies (Han et al., 2000). The potentially significant problem that might limit the utility of this system, at least in Drosophila, is "non-specific" background expression in a certain tissue regardless of the driver promoter used. Fly enhancer detector lines made with GAL4-ER revealed a driver-independent expression pattern in the neurolema (Han et al., 2000; Roman et al., 2001). Other drawbacks of this system, when used in transgenic animals, include the possible activation of target gene by endogenous estrogen and/or deregulation of endogenous estrogen-responsive genes by administration of additional estrogen (Burcin et al., 1998).

\section{Progesterone-receptor-GAL4 fusion based system}

The progesterone-dependent chimeric transcription factor (GL-VP, Burcin et al., 1998; DeMayo and Tsai, 2001; Wang et al., 1994) consists of the GAL4 DNA-binding domain fused to the ligand binding domain of a mutated human progesterone receptor and the VP16 activation domain. This mutated ligand binding domain with a truncated 
carboxy terminus alters the ligand-binding specificity of the original receptor so that it only binds to the synthetic progesterone antagonist RU486 (also known as mifepristone) and is no longer capable of binding to progesterone (Vegeto et al., 1992). Consequently, only in the presence of RU486, but not any endogenous hormones present in animals, the chimeric GL-VP with this modified progesterone-binding domain is relieved from cytoplasmic sequestration in a heat shock protein complex, travels to the nucleus, and binds to genes linked to UAS, resulting in efficient ligand-inducible transactivation of the target gene. In addition, GL-VP responds to RU486 at a sub-physiological concentration that is significantly lower than that required for antagonizing endogenous progesterone or glucocorticoid activity. The utility of this system was initially demonstrated in cultured mammalian cells, with a 30-50-fold activation of a luciferase reporter in the presence of RU486 (Wang et al., 1994). Successful application of this regulatory system also has been reported in transgenic models including mice (Burcin et al., 1999; Pierson et al., 2000; Wang et al., 1999; Wang et al., 1997) and, most recently, Drosophila (Osterwalder et al., 2001; Roman et al., 2001).

\section{Applications in animal models}

The progesterone-inducible binary system has been used in several different tissues of transgenic mice to drive the expression of various gene products including human growth hormone (hGH; Wang et al., 1997), inhibin A (Pierson et al., 2000), and transforming growth factor $\beta$ (TGFß; Wang et al., 1999). The resultant transgenic animals showed phenotypes resulting from the rapid induction of transgenes in response to RU486. The GL-VP has since been modified by replacing the VP16 activation domain 
with the activation domain of the less immunogenic human p65 protein, a component of the NFאB complex, in order to preclude a potential immune response caused by the viral VP16 activation domain, specially in mammalian tissues (Burcin et al., 1999; DeMayo and Tsai, 2001). This new regulator, the GL-p65 (GeneSwitch), has been used with an adenoviral vector-mediated delivery system to express the $\mathrm{hGH}$ gene in infected mice (Burcin et al., 1999) and to induce the expression of fibroblast growth factor 3 (FGF3) in the lungs of transgenic mice (Zhao et al., 2001). RU486-dependent phenotypes were obtained, which were reversible after withdrawal of RU486. Most recently, the applicability of the GeneSwitch for conditional expression of transgene in Drosophila has also been reported (Osterwalder et al., 2001; Roman et al., 2001). Osterwalder et al. (2001) have shown that using defined promoters, GFP reporter protein expression could be detected $5 \mathrm{hr}$ after systemic administration of RU486 by either direct feeding or "larval bathing", (soaking larvae in an RU486-containing solution for a short period of time). Transgene expression levels were found to be RU486 dose-dependent with little background expression in the absence of RU486. In addition, they were able to regulate the expression of genetically modified channels in larval muscles and neurons, resulting in a change of the neuromuscular activity of bodywall muscles in an RU486-dependent manner. Roman et al. (2001) have used a similar approach to ablate fat body tissue in adult females through the induced expression of diphtheria toxin using tissue-specific RU486-responsive enhancer detector lines, thus validating the GeneSwitch as an effective conditional transgene regulatory system.

The RU486-inducible regulatory system described in this section represents an invaluable tool for the controlled manipulation of transgenes. When combined with other 
strategies such as a dominant negative approach, this system offers a variety of applications to study functions of gene products at specific developmental stages in specific tissues during development, which was not possible previously in Xenopus.

Genes implicated in eye development often express and function in other tissues including anterior brain and the eye at multiple stages such that constitutive mutations in these genes often cause early embryonic lethality or pleiotropy precluding study of later phenotype. This obscures the primary roles for a gene product that function in a particular process at a particular time. My motivation to develop an inducible system allowing temporal control of transgene expression was to express these genes (or their derivatives) in the developing eye primordia at desired developmental stages to attempt to circumvent these problems. New transgenic techniques and the availability of characterized promoters allowing the delivery of GAL4 (and UAS) constructs to a developmental pattern of interest also motivated me to explore the use of a hormone-dependent GAL4 and to develop the inducible binary GAL4/UAS system using $X$. tropicalis as a potentially valuable new genetic system. I have utilized this system to study an important biological problem, the function of the Otx2 gene product during ocular development. 


\section{References}

\section{Acampora, D., Mazan, S., Lallemand, Y., Avantaggiato, V., Maury, M., Simeone, A.}

and Brulet, P. (1995). Forebrain and midbrain regions are deleted in Otx2-/- mutants due to a defective anterior neuroectoderm specification during gastrulation. Development 121, 3279-90.

Amaya, E., Offield, M. F. and Grainger, R. M. (1998). Frog genetics: Xenopus tropicalis jumps into the future. Trends in Genetics 14, 253-5.

Ang, S. L., Jin, O., Rhinn, M., Daigle, N., Stevenson, L. and Rossant, J. (1996). A targeted mouse Otx2 mutation leads to severe defects in gastrulation and formation of axial mesoderm and to deletion of rostral brain. Development 122, 243-52.

Ashery-Padan, R. and Gruss, P. (2001). Pax6 lights-up the way for eye development. Curr Opin Cell Biol 13, 706-14.

Ashery-Padan, R., Marquardt, T., Zhou, X. and Gruss, P. (2000a). Pax6 activity in the lens primordium is required for lens formation and for correct placement of a single retina in the eye. Genes \& Development 14, 2701-11.

Ashery-Padan, R., Marquardt, T., Zhou, X. and Gruss, P. (2000b). Pax6 activity in the lens primordium is required for lens formation and for correct placement of a single retina in the eye. Genes Dev 14, 2701-11.

Baim, S. B., Labow, M. A., Levine, A. J. and Shenk, T. (1991). A chimeric mammalian transactivator based on the lac repressor that is regulated by temperature and isopropyl beta-D-thiogalactopyranoside. Proceedings of the National Academy of Sciences of the United States of America 88, 5072-6. 
Bello, B., Resendez-Perez, D. and Gehring, W. J. (1998). Spatial and temporal targeting of gene expression in Drosophila by means of a tetracycline-dependent transactivator system. Development 125, 2193-202.

Bieschke, E. T., Wheeler, J. C. and Tower, J. (1998). Doxycycline-induced transgene expression during Drosophila development and aging. Mol Gen Genet 258, 571-9.

Blitz, I. L. and Cho, K. W. (1995). Anterior neurectoderm is progressively induced during gastrulation: the role of the Xenopus homeobox gene orthodenticle. Development Supplement 121, 993-1004.

Brand, A. H. and Perrimon, N. (1993). Targeted gene expression as a means of altering cell fates and generating dominant phenotypes. Development 118, 401-15.

Braselmann, S., Graninger, P. and Busslinger, M. (1993). A selective transcriptional induction system for mammalian cells based on Gal4-estrogen receptor fusion proteins. Proceedings of the National Academy of Sciences of the United States of America 90, $1657-61$.

Broccoli, V., Boncinelli, E. and Wurst, W. (1999). The caudal limit of Otx2 expression positions the isthmic organizer. Nature 401, 164-8.

Bronchain, O. J., Hartley, K. O. and Amaya, E. (1999). A gene trap approach in Xenopus. Current Biology 9, 1195-1198.

Burcin, M. M., BW, O. M. and Tsai, S. Y. (1998). A regulatory system for target gene expression. Front Biosci 3, c1-7.

Burcin, M. M., Schiedner, G., Kochanek, S., Tsai, S. Y. and O'Malley, B. W. (1999). Adenovirus-mediated regulable target gene expression in vivo. Proceedings of the National Academy of Sciences of the United States of America 96, 355-60. 
Callaerts, P., Halder, G. and Gehring, W. J. (1997). PAX-6 in development and evolution. Annual Review of Neuroscience 20, 483-532.

Chae, J., Zimmerman, L. B. and Grainger, R. M. (2002). Inducible control of tissuespecific transgene expression in Xenopus tropicalis transgenic lines. Mech Dev 117, 23541.

Chisholm, A. D. and Horvitz, H. R. (1995). Patterning of the Caenorhabditis elegans head region by the Pax-6 family member vab-3. Nature 377, 52-5.

Chow, R. L., Altmann, C. R., Lang, R. A. and Hemmati-Brivanlou, A. (1999). Pax6 induces ectopic eyes in a vertebrate. Development 126, 4213-22.

Cronin, C. A., Gluba, W. and Scrable, H. (2001). The lac operator-repressor system is functional in the mouse. Genes Dev 15, 1506-17.

D'Avino, P. P. and Thummel, C. S. (1999). Ectopic expression systems in Drosophila. Methods Enzymol 306, 129-42.

DeMayo, F. J. and Tsai, S. Y. (2001). Targeted gene regulation and gene ablation. Trends Endocrinol Metab 12, 348-53.

Deuschle, U., Pepperkok, R., Wang, F. B., Giordano, T. J., McAllister, W. T., Ansorge, W. and Bujard, H. (1989). Regulated expression of foreign genes in mammalian cells under the control of coliphage T3 RNA polymerase and lac repressor. Proceedings of the National Academy of Sciences of the United States of America 86, $5400-4$.

Dujon, B., Alexandraki, D., Andre, B., Ansorge, W., Baladron, V., Ballesta, J. P., Banrevi, A., Bolle, P. A., Bolotin-Fukuhara, M., Bossier, P. et al. (1994). Complete DNA sequence of yeast chromosome XI. Nature 369, 371-8. 
Finkelstein, R., Smouse, D., Capaci, T. M., Spradling, A. C. and Perrimon, N. (1990). The orthodenticle gene encodes a novel homeo domain protein involved in the development of the Drosophila nervous system and ocellar visual structures. Genes Dev 4, 1516-27.

Gossen, M., Bonin, A. L. and Bujard, H. (1993). Control of gene activity in higher eukaryotic cells by prokaryotic regulatory elements. Trends Biochem Sci 18, 471-5. Gossen, M., Bonin, A. L., Freundlieb, S. and Bujard, H. (1994). Inducible gene expression systems for higher eukaryotic cells. Curr Opin Biotechnol 5, 516-20. Gossen, M., Freundlieb, S., Bender, G., Muller, G., Hillen, W. and Bujard, H. (1995). Transcriptional activation by tetracyclines in mammalian cells. Science 268, 1766-9.

Grainger, R. M. (1996). New perspectives on embryonic lens induction. Seminars in Cell and Developmental Biology 7, 149-55.

Halder, G., Callaerts, P. and Gehring, W. J. (1995). Induction of ectopic eyes by targeted expression of the eyeless gene in Drosophila. Science 267, 1788-92.

Han, D. D., Stein, D. and Stevens, L. M. (2000). Investigating the function of follicular subpopulations during Drosophila oogenesis through hormone-dependent enhancertargeted cell ablation. Development 127, 573-83.

Hartley, K. O., Hardcastle, Z., Friday, R. V., Amaya, E. and Papalopulu, N. (2001). Transgenic Xenopus embryos reveal that anterior neural development requires continued suppression of BMP signaling after gastrulation. Developmental Biology 238, 168-84. 
Hemmati-Brivanlou, A. and Melton, D. A. (1992). A truncated activin receptor inhibits mesoderm induction and formation of axial structures in Xenopus embryos. Nature 359, 609-14.

Henry, J. J. and Grainger, R. M. (1987). Inductive interactions in the spatial and temporal restriction of lens-forming potential in embryonic ectoderm of Xenopus laevis. Developmental Biology 124, 200-14.

Henry, J. J. and Grainger, R. M. (1990). Early tissue interactions leading to embryonic lens formation in Xenopus laevis. Developmental Biology 141, 149-163.

Herskowitz, I. (1987). Functional inactivation of genes by dominant negative mutations. Nature 329, 219-222.

Hill, R. E., Favor, J., Hogan, B. L., Ton, C. C., Saunders, G. F., Hanson, I. M., Prosser, J., Jordan, T., Hastie, N. D. and van Heyningen, V. (1991). Mouse small eye results from mutations in a paired-like homeobox-containing gene. Nature 354, 522-5.

Hirsch, N. and Grainger, R. M. (2000). Induction of the lens. Results \& Problems in Cell Differentiation 31, 51-68.

Hirsch, N. and Harris, W. A. (1997). Xenopus Pax-6 and retinal development. Journal of Neurobiology 32, 45-61.

Hirsch, N., Zimmerman, L. B. and Grainger, R. M. (2002a). Xenopus, the next generation: X. Tropicalis genetics and genomics. Developmental Dynamics 225, 422-33. Hirsch, N., Zimmerman, L. B., Gray, J., Chae, J., Curran, K. L., Fisher, M., Ogino, H. and Grainger, R. M. (2002b). Xenopus tropicalis transgenic lines and their use in the study of embryonic induction. Developmental Dynamics 225, 522-35. 
Hollenberg, S. M., Cheng, P. F. and Weintraub, H. (1993). Use of a conditional MyoD transcription factor in studies of MyoD trans-activation and muscle determination. Proceedings of the National Academy of Sciences of the United States of America 90, 8028-32.

Isaacs, H. V., Andreazzoli, M. and Slack, J. M. (1999). Anteroposterior patterning by mutual repression of orthodenticle and caudal-type transcription factors. Evol Dev 1, 14352.

Jacob, F. and Monod, J. (1961). Genetic regulatory mechanisms in the synthesis of proteins. J Mol Biol 3, 318-56.

Jordan, T., Hanson, I., Zaletayev, D., Hodgson, S., Prosser, J., Seawright, A., Hastie, N. and van Heyningen, V. (1992). The human PAX6 gene is mutated in two patients with aniridia. Nature Genetics 1, 328-332.

Khoka, M., Chung, C., Bustamante, E. L., Gaw, L., Trott, K., Yeh, J., Taverner, N., Amaya, E., Smith, J. C., Papalopulu, N. et al. (2002). Techniques and probes for the study of Xenopus tropicalis development. Developmental Dynamics in press.

Khokha, M. K., Chung, C., Bustamante, E. L., Gaw, L. W., Trott, K. A., Yeh, J., Lim, N., Lin, J. C., Taverner, N., Amaya, E. et al. (2002). Techniques and probes for the study of Xenopus tropicalis development. Dev Dyn 225, 499-510.

Kolm, P. J. and Sive, H. L. (1995). Efficient hormone-inducible protein function in Xenopus laevis. Developmental Biology 171, 267-72.

Kroll, K. L. and Amaya, E. (1996). Transgenic Xenopus embryos from sperm nuclear transplantations reveal FGF signaling requirements during gastrulation. Development 122, 3173-83. 
Krotoski, D. M., Reinschmidt, D. C. and Tompkins, R. (1985). Developmental mutants isolated from wild-caught Xenopus laevis by gynogenesis and inbreeding. $J$ Exp Zool 233, 443-9.

Lewis, E. B. (1978). A gene complex controlling segmentation in Drosophila. Nature 276, 565-70.

Mallamaci, A., Di Blas, E., Briata, P., Boncinelli, E. and Corte, G. (1996). OTX2 homeoprotein in the developing central nervous system and migratory cells of the olfactory area. Mech Dev 58, 165-78.

Mansuy, I. M., Winder, D. G., Moallem, T. M., Osman, M., Mayford, M., Hawkins, R. D. and Kandel, E. R. (1998). Inducible and reversible gene expression with the rtTA system for the study of memory. Neuron 21, 257-65.

Marquardt, T., Ashery-Padan, R., Andrejewski, N., Scardigli, R., Guillemot, F. and Gruss, P. (2001). Pax6 is required for the multipotent state of retinal progenitor cells. Cell 105, 43-55.

Martinez-Morales, J. R., Signore, M., Acampora, D., Simeone, A. and Bovolenta, P. (2001). Otx genes are required for tissue specification in the developing eye.

Development 128, 2019-30.

Mathers, P. H., Grinberg, A., Mahon, K. A. and Jamrich, M. (1997). The Rx homeobox gene is essential for vertebrate eye development. Nature 387, 603-7.

Miklos, G. L. and Rubin, G. M. (1996). The role of the genome project in determining gene function: insights from model organisms. Cell 86, 521-9. 
Moore, S., Keller, R. and Koehl, M. (1995). The dorsal involuting marginal zone stiffens anisotropically during its convergent extension in the gastrula of Xenopus laevis. Development 121, 3131-3140.

Morozov, A., Kellendonk, C., Simpson, E. and Tronche, F. (2003). Using conditional mutagenesis to study the brain. Biol Psychiatry 54, 1125-33.

Myers, G. L. and Sadler, J. R. (1971). Mutational inversion of control of the lactose operon of Escherichia coli. J Mol Biol 58, 1-28.

Nishida, A., Furukawa, A., Koike, C., Tano, Y., Aizawa, S., Matsuo, I. and Furukawa, T. (2003). Otx2 homeobox gene controls retinal photoreceptor cell fate and pineal gland development. Nat Neurosci 6, 1255-63.

Noramly, S. and Grainger, R. M. (2002). Determination of the embryonic inner ear. $J$ Neurobiol 53, 100-28.

Offield, M. F., Hirsch, N. and Grainger, R. M. (2000). The development of Xenopus tropicalis transgenic lines and their use in studying lens developmental timing in living embryos. Development 127, 1789-97.

Ogino, H. and Yasuda, K. (2000). Sequential activation of transcription factors in lens induction. Development Growth \& Differentiation 42, 437-48.

Ornitz, D. M., Moreadith, R. W. and Leder, P. (1991). Binary system for regulating transgene expression in mice: targeting int-2 gene expression with yeast GAL4/UAS control elements. Proceedings of the National Academy of Sciences of the United States of America 88, 698-702. 
Osterwalder, T., Yoon, K. S., White, B. H. and Keshishian, H. (2001). A conditional tissue-specific transgene expression system using inducible GAL4. Proceedings of the National Academy of Sciences of the United States of America 98, 12596-601.

Pannese, M., Polo, C., Andreazzoli, M., Vignali, R., Kablar, B., Barsacchi, G. and Boncinelli, E. (1995). The Xenopus homologue of Otx2 is a maternal homeobox gene that demarcates and specifies anterior body regions. Development 121, 707-20.

Parkhurst, S. M. and Ish-Horowicz, D. (1991). Mis-regulating segmentation gene expression in Drosophila. Development 111, 1121-35.

Pichaud, F. and Desplan, C. (2002). Pax genes and eye organogenesis. Curr Opin Genet Dev 12, 430-4.

Pierson, T. M., Wang, Y., DeMayo, F. J., Matzuk, M. M., Tsai, S. Y. and Omalley, B. W. (2000). Regulable expression of inhibin A in wild-type and inhibin alpha null mice. Molecular Endocrinology 14, 1075-85.

Quiring, R., Walldorf, U., Kloter, U. and Gehring, W. J. (1994). Homology of the eyeless gene of Drosophila to the Small eye gene in mice and Aniridia in humans [see comments]. Science 265, 785-9.

Robinson, M. L., Macmillancrow, L. A., Thompson, J. A. and Overbeek, P. A. (1995). Expression of a Truncated Fgf Receptor Results in Defective Lens Development in Transgenic Mice. Development 121, 3959-3967.

Roman, G., Endo, K., Zong, L. and Davis, R. L. (2001). P[Switch], a system for spatial and temporal control of gene expression in Drosophila melanogaster. Proceedings of the National Academy of Sciences of the United States of America 98, 12602-7. 
Rorth, P. (1996). A modular misexpression screen in Drosophila detecting tissuespecific phenotypes. Proceedings of the National Academy of Sciences of the United States of America 93, 12418-22.

Rorth, P., Szabo, K., Bailey, A., Laverty, T., Rehm, J., Rubin, G. M., Weigmann, K., Milan, M., Benes, V., Ansorge, W. et al. (1998). Systematic gain-of-function genetics in Drosophila. Development 125, 1049-57.

Ryan, A. and Scrable, H. (2004). Visualization of the dynamics of gene expression in the living mouse. Mol Imaging 3, 33-42.

Saha, M. S., Spann, C. L. and Grainger, R. M. (1989). Embryonic lens induction: more than meets the optic vesicle. Cell Differ Dev 28, 153-71.

Schedl, A., Ross, A., Lee, M., Engelkamp, D., Rashbass, P., van Heyningen, V. and Hastie, N. D. (1996). Influence of PAX6 gene dosage on development: overexpression causes severe eye abnormalities. Cell 86, 71-82.

Scheer, N. and Camnos-Ortega, J. A. (1999). Use of the Gal4-UAS technique for targeted gene expression in the zebrafish. Mech Dev 80, 153-8.

Schneuwly, S., Klemenz, R. and Gehring, W. J. (1987). Redesigning the body plan of Drosophila by ectopic expression of the homoeotic gene Antennapedia. Nature 325, 8168.

Shockett, P., Difilippantonio, M., Hellman, N. and Schatz, D. G. (1995). A modified tetracycline-regulated system provides autoregulatory, inducible gene expression in cultured cells and transgenic mice. Proceedings of the National Academy of Sciences of the United States of America 92, 6522-6. 
Shockett, P. E. and Schatz, D. G. (1996). Diverse strategies for tetracycline-regulated inducible gene expression. Proceedings of the National Academy of Sciences of the United States of America 93, 5173-6.

Simeone, A., Acampora, D., Mallamaci, A., Stornaiuolo, A., MR, D. A., Nigro, V. and Boncinelli, E. (1993). A vertebrate gene related to orthodenticle contains a homeodomain of the bicoid class and demarcates anterior neuroectoderm in the gastrulating mouse embryo. EMBO Journal 12, 2735-47.

Smith, W. C. and Harland, R. M. (1991). Injected Xwnt-8 RNA acts early in Xenopus embryos to promote formation of a vegetal dorsalizing center. Cell 67, 753-765.

Smith, W. C. and Harland, R. M. (1992). Expression cloning of noggin, a new dorsalizing factor localized to the Spemann organizer in Xenopus embryos. Cell 70, 82940.

Stebbins, M. J., Urlinger, S., Byrne, G., Bello, B., Hillen, W. and Yin, J. C. (2001). Tetracycline-inducible systems for Drosophila. Proceedings of the National Academy of Sciences of the United States of America 98, 10775-80.

Stebbins, M. J. and Yin, J. C. (2001). Adaptable doxycycline-regulated gene expression systems for Drosophila. Gene 270, 103-11.

Sulston, J., Du, Z., Thomas, K., Wilson, R., Hillier, L., Staden, R., Halloran, N., Green, P., Thierry-Mieg, J., Qiu, L. et al. (1992). The C. elegans genome sequencing project: a beginning. Nature 356, 37-41.

Ton, C. C., Hirvonen, H., Miwa, H., Weil, M. M., Monaghan, P., Jordan, T., van Heyningen, V., Hastie, N. D., Meijers-Heijboer, H., Drechsler, M. et al. (1991). 
Positional cloning and characterization of a paired box- and homeobox-containing gene from the aniridia region. Cell 67, 1059-74.

Vegeto, E., Allan, G. F., Schrader, W. T., Tsai, M. J., McDonnell, D. P. and

O'Malley, B. W. (1992). The mechanism of RU486 antagonism is dependent on the conformation of the carboxy-terminal tail of the human progesterone receptor. Cell 69, 703-13.

Viczian, A. S., Vignali, R., Zuber, M. E., Barsacchi, G. and Harris, W. A. (2003). XOtx $5 \mathrm{~b}$ and XOtx2 regulate photoreceptor and bipolar fates in the Xenopus retina. Development 130, 1281-94.

Vogel, G. (1999). Frog is a prince of a new model organism. Science 285, 25.

Walther, C. and Gruss, P. (1991). Pax-6, a murine paired box gene, is expressed in the developing CNS. Development 113, 1435-49.

Wang, X. J., Liefer, K. M., Tsai, S., O'Malley, B. W. and Roop, D. R. (1999).

Development of gene-switch transgenic mice that inducibly express transforming growth factor beta1 in the epidermis. Proceedings of the National Academy of Sciences of the United States of America 96, 8483-8.

Wang, Y., DeMayo, F. J., Tsai, S. Y. and O'Malley, B. W. (1997). Ligand-inducible and liver-specific target gene expression in transgenic mice. Nat Biotechnol 15, 239-43. Wang, Y., O'Malley, B. W., Jr., Tsai, S. Y. and O'Malley, B. W. (1994). A regulatory system for use in gene transfer. Proceedings of the National Academy of Sciences of the United States of America 91, 8180-4.

Wheeler, G. N., Hamilton, F. S. and Hoppler, S. (2000). Inducible gene expression in transgenic Xenopus embryos. Current Biology 10, 849-52. 
Wilson, P. A., Oster, G. and Keller, R. (1989). Cell rearrangement and segmentation in Xenopus: direct observation of cultured explants. Development - Supplement 105, 155 66. Zhang, L., El-Hodiri, H. M., Ma, H. F., Zhang, X., Servetnick, M., Wensel, T. G. and Jamrich, M. (2003). Targeted expression of the dominant-negative FGFR4a in the eye using Xrx1A regulatory sequences interferes with normal retinal development. Development 130, 4177-86.

Zhang, Y. and Emmons, S. W. (1995). Specification of sense-organ identity by a Caenorhabditis elegans Pax-6 homologue. Nature 377, 55-9.

Zhao, B., Chua, S. S., Burcin, M. M., Reynolds, S. D., Stripp, B. R., Edwards, R. A., Finegold, M. J., Tsai, S. Y. and DeMayo, F. J. (2001). Phenotypic consequences of lung-specific inducible expression of FGF-3. Proceedings of the National Academy of Sciences of the United States of America 98, 5898-903.

Zygar, C. A., Cook, T. L. and Grainger, R. M., Jr. (1998). Gene activation during early stages of lens induction in Xenopus. Development 125, 3509-19. 


\section{Chapter 2:}

Development of hormone-inducible GAL4/UAS system in Xenopus: temporal control of tissue-specific reporter transgene expression in X. tropicalis embryos

Note regarding Chapter 2:

A part of Chapter 2 has been published as

Chae, J., Zimmerman, L. B. and Grainger, R. M. (2002). Inducible control of tissuespecific transgene expression in Xenopus tropicalis transgenic lines. Mechanism of Development 117, 235-241.

L. B. Zimmerman generated transgenic pRx-GALPR and UAS-ECFP founder animals and did preliminary tests of RU486 treatment. 


\section{Abstract}

Analysis of gene function in vertebrates is facilitated by gain-of-function studies, such as injection of synthetic mRNA in amphibian embryos. This approach is hampered by lack of spatial and temporal control of expression of the introduced gene product. An additional level of control is obtained by nuclear-transfer-mediated transgenesis, but functional analyses are complicated by variability and background abnormalities in primary transgenic embryos.

The GAL4/UAS system permits establishment of stable lines and elimination of nuclear-transfer-associated abnormalities, through generation of separate UAS-“effector" and GAL4 "transactivator" transgenic lines. When the GAL4 DNA-binding domain is combined with a steroid hormone ligand-binding domain, this system allows full temporal regulation of transgene expression by introduction of an exogenous steroid analogue, the progesterone antagonist RU486.

By crossing stable Xenopus tropicalis transgenic lines, one bearing a UAS-GFP reporter construct, and the other with a GAL4-progesterone receptor fusion driven by a retina-specific promoter, reporter expression in the resulting embryos was induced in an RU486 dose-dependent and tissue-specific manner. Robust transgene expression could be detected within 2.5 hours after application of RU486, with very low background expression in the absence of the ligand.

These results suggest that the inducible binary system, in which the target gene expression can be controlled in a stage- and tissue-specific pattern, should be readily applicable for gene function studies at all stages of development. 


\section{Introduction}

In Xenopus studies, over- or misexpression of a gene product by mRNA injection is the most common way to analyze the function of a cloned gene. However, misexpression-associated pleiotropy or lethality often masks analysis of later developmental processes. The recently-developed frog transgenesis technique (Offield et al., 2000) permits the expression of a gene product in a tissue-specific manner by delivering the gene product under the control of a defined promoter (Hartley et al., 2001). Analysis of the consequences of transgene expression in primary transgenics is, however, often complicated by position effects, variability in expression levels, and background abnormalities associated with nuclear transfer. These problems can be circumvented by establishing stable transgenic lines, but only where transgene expression is non-lethal.

To overcome these difficulties, I have applied the GAL4/UAS system extensively utilized in the fly (Brand and Perrimon, 1993). This system divides the target gene (UASeffector) from transcriptional activation elements (GAL4 transactivator) in two separate transgenic lines. In an effector line the target gene, under the control of GAL4 binding

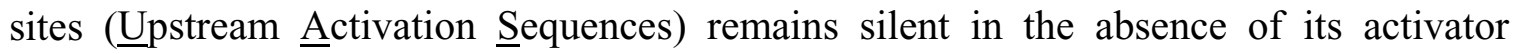
GAL4, ensuring that these transgenic founders are viable. Only in the progeny of a cross of a UAS-effector line to a GAL4 transactivator line is the target gene expressed.

This system can also provide an additional level of temporal control of transgene expression by fusing the GAL4 binding domain with a steroid receptor ligand-binding domain (Wang et al., 1994). The transcriptional activity of this chimeric GAL4 protein is regulated by appropriate steroid hormones or chemically related exogenous compound. 
The GAL4 fusion protein utilized in this study is based on the modified human progesterone ligand-binding domain (Burcin et al., 1998). This mutated ligand binding domain with the altered specificity only binds to the progesterone antagonist RU486, resulting in efficient ligand-inducible activation of the target gene linked to UAS only in the presence of RU486, but not any endogenous hormones including progesterone itself (Vegeto et al., 1992). The utility of the system was initially tested in cultured mammalian cells and transfected cells showed rapid induction of a target gene in an RU486dependent manner (Burcin et al., 1999; Wang et al., 1994). This inducible binary regulatory system also has been successfully used to drive the expression of various gene products in transgenic models including mice (Burcin et al., 1999; Pierson et al., 2000; Wang et al., 1999; Wang et al., 1997) and, most recently, Drosophila (Osterwalder et al., 2001; Roman et al., 2001).

In this study, I tested the GAL4/UAS system in the pipid frog Xenopus tropicalis, which has emerged as a complementary system to its close relative, Xenopus laevis (Amaya et al., 1998). X. tropicalis shares many of the advantages of X. laevis, but is more suited for multigeneration genetic experiments by virtue of its short generation time and smaller diploid genome.

Here I show that, using the transgenic activator and effector lines I established, the expression of UAS-target reporter gene can be efficiently activated by a ligandinducible GAL4 in response to RU486. 


\section{Materials and methods}

\section{DNA constructs and Generation of transgenic lines}

A $2.2 \mathrm{~kb} 5$ '-upstream region of the $R x$ (retina homobox ; Mathers et al., 1997) gene was obtained by screening a Lambda FIX-II Xenopus tropicalis genomic library (constructed by Lisa Brunet and Richard Harland) with a Xenopus laevis Rx cDNA probe containing the $5^{\prime}$ untranslated region. The $\mathrm{pRx}-\mathrm{GFP}$ construct was generated by subcloning this $2.2 \mathrm{~kb}$ region of the $X$. tropicalis $R x$ promoter into pEGFP-1 vector (Clontech). This construct was linearized with AflII for making transgenic animals. The pRx-GALPR activator construct was generated by deleting an Asp718-NotI/blunt fragment containing the EGFP coding sequences in pRx-GFP, and replacing them with an Asp718-BamHI/blunt fragment containing the chimeric GAL4-VP16-mutant progesterone receptor ligand-binding domain isolated from pGL-VP (Wang et al., 1994). To generate a template plasmid DNA to make an in situ hybridization probe to detect GALPR transcript, an Asp718-xhoI fragment containing GAL4-VP16 from the pRxGALPR plasmid was inserted to Asp718-XhoI-digested pCS2 vector. This resulted in pCS2-GAL4-VP16R in which the GAL4-VP16 coding sequence is reversed in order to take advantage of using a stronger SP6 RNA polymerase for in vitro transcription. To construct the bifunctional UAS-ECFP plasmid, the cardiac actin promoter in pCARGFP (gift of E. Amaya) (Kroll and Amaya, 1996) was removed and cloned into plasmid pDsRed1 (Clontech), creating pCAR-RFP construct. An AflII fragment of the resulting construct containing the CAR-RFP cassette then was inserted into plasmid pECFP-1 (Clontech), resulting in double color reporter pBicolor in which the expression of RFP is 
driven by the cardiac actin promoter and any promoter can be cloned in front of the ECFP coding sequences. Finally, the HindIII-SmaI fragment containing five copies of the GAL4 consensus binding sites (UAS) linked to the $h s p 70$ TATA box was isolated from plasmid GUAS-GFP3 (gift from E. Amaya) (Bronchain et al., 1999) derived from pUAST (Brand and Perrimon, 1993) and subcloned into plasmid pBicolor, creating UASECFP (UAS-ECFP-pCAR-RFP). Transgenic lines used in this study were generated as previously described (Offield et al., 2000) with some modifications (see also Transgenesis in $X$ tropicalis at $X$. tropicalis website; http://minerva.acc.Virginia.EDU/ develbio/trop/).

\section{GAL4 mRNA injections}

A male transgenic UAS-ECFP founder was outcrossed to a wild-type counterpart. One blastomere of two-cell stage embryos of this cross was injected with 1 or $10 \mathrm{pg}$ of capped synthetic GAL4 RNA transcribed in vitro using SP6 polymerase from a NotIlinearized pCS2/GAL4 template (gift of E. Amaya) encoding native GAL4 DNA binding and activation domains. Resulting embryos were then analyzed for GAL4-induced ECFP expression and the expression of RFP in the muscle.

\section{RU486 treatments and Western analysis}

For experiments involving treatment with RU486 (BIOMOL Research Labs, Inc.), embryos expressing activator and effector transgenes were produced by crossing a low background F1 transgenic effector animal (see Results) to a GALPR activator founder. Embryos were cultured in 0 (solvent control), 5, or $25 \mathrm{nM}$ RU486 freshly 
diluted in $0.1 \times$ MBS with $0.1 \%$ BSA from a stock initially dissolved in DMSO at 25 $\mathrm{mg} / \mathrm{ml}$ and subsequently diluted to $5.8 \mu \mathrm{M}$ in $1 \mathrm{M}$ acetic acid. For kinetics assays, embryos were cultured in $25 \mathrm{nM}$ RU486 from st 12/13 onward and fixed after 2.5, 5.0, 7.5, and $10 \mathrm{hr}$, respectively, for further in situ hybridization analysis. For the test of DMSO (CAS no. 67-68-5; Sigma Aldrich Chemical Co., Inc.) effects, a $100 \%$ DMSO stock was directly added to a RU486-containing culture medium $(0.1 \times$ MBS with $0.1 \%$ BSA with 0 or $25 \mathrm{nM}$ RU486) to generate the following final DMSO concentrations $(\mathrm{v} / \mathrm{v}): 0 \%, 0.5 \%, 1.0 \%$, and $1.5 \%$. An insignificant concentration of DMSO was present in all samples, in addition, as a result of dissolving RU486 in this solvent, which was diluted approximately $2.3 \times 10^{6}$ times in the final culture medium. For Western analysis, eye extracts were prepared by sonicating head tissue consisting primarily of eye rudiment dissected from RU486-treated embryos in a standard NP-40 lysis buffer. Protein levels were quantified by Bradford assay (Bio-Rad) and $5 \mu \mathrm{g}$ of extracted protein was separated on a $12 \%$ SDS/PAGE gel and transferred onto a nitrocellulose membrane (Osmonics, Inc.). The blot was probed with the Living Colors A.v. Peptide antibody (Clontech) recognizing EGFP derivatives and an Anti-Actin antibody (Sigma) diluted in 1:100 or 1:1000, respectively. The signals were visualized by ECL (Amersham Pharmacia). Finally, intensities of resulting bands were determined and calculated using the Kodak Digital Science Image Station 440 CF (NEN).

\section{In situ hybridization}

In situ hybridization was essentially carried out according to the procedure of Harland (1991) as modified in Sive et al. (2000). The following antisense RNA probes 
labeled with digoxigenin (Roche) were generated using the corresponding cDNAs as templates, by linearization of with the indicated restriction enzymes and in vitro transcribed with the indicated RNA polymerase; $R x$ probe, $X r x-1$ with $B a m H I$ and T7 RNA polysmerase; GAL4 probe, pCS2-GAL4-VP16R (see DNA constructs above) with Asp718 and SP6 RNA polymerase; GFP probe, pCS2-EGFP (Clontech) with EcoRI and T7 RNA polymerase. RNA probes were revealed using BM purple (Roche) as the substrate for the alkaline phosphatase.

\section{Histology}

Following whole mount in situ hybridization, embryos were postfixed overnight at room temperature in MEMFA (0.1M MOPS, $2 \mathrm{mM}$ EDTA, $1 \mathrm{mM} \mathrm{MgSO}_{4}, 3.7 \%$ formaldehyde) or Bouin's fix ( $1 \%$ picric acid, $9.25 \%$ formaldehyde, $0.05 \%$ glacial acetic acid) and examined as wholemounts in phosphate-buffered saline (PBS). Some embryos were dehydrated, embedded in Paraplast (Oxford Labware) and sectioned on a microtome at 10-15 $\mu \mathrm{m}$. Sections were coverslipped in Permount (Fisher) before photomicrography. For fluorescent histochemistry of cryosections, embryos were fixed in MEMFA (Harland, 1991) for $1-1.5 \mathrm{hr}$ at room temperature or overnight at $4^{\circ} \mathrm{C}$ and stored in ethanol at $20^{\circ} \mathrm{C}$. They were rehydrated in graded ethanols and infiltrated in $30 \%$ sucrose in PBS for 1 hour at room temperature and embedded in OCT compound (Tissue-Tek, Sakura Finetechnical Co., Ltd.), frozen and sectioned at 10-15 $\mu \mathrm{m}$ on a Leica CM3050 cryostat. 


\section{Photomicroscopy}

Images of transgenic embryos were obtained using a Zeiss Stemi Epicfluorescence stereoscope with GFP 500 and rhodamine filter sets. Non-fluorescent whole-mount images were obtained without the filter sets. Sectioned samples were photographed on a Zeiss Axioskop microscope. Images were captured using a Dage MTI 330 cooled CCD camera or a Carl Zeiss AxioCam MRc 5 camera.

\section{Results}

\section{Injected GAL4 RNA activates the transcription of transgenic UAS effector gene}

I have generated $X$. tropicalis effector lines carrying a UAS-ECFP construct, in which the gene for a green fluorescent protein (GFP) variant, ECFP (Clontech), is under the control of GAL4 binding motifs. This construct also contains a second reporter cassette (pCAR-RFP) with the cardiac actin promoter driving Red Fluorescent Protein expression in the somites (Hartley et al., 2001; Kroll and Amaya, 1996) to identify embryos carrying the transgene (Fig. 1A).

To establish the utility of the UAS-ECFP line I first tested whether exogenous GAL4 can activate transcription in stable UAS-ECFP transgenic embryos by transient expression assays. A male transgenic founder was crossed with a wild-type female, and resulting embryos were then injected in one blastomere with 1 or $10 \mathrm{pg}$ of synthetic GAL4 mRNA at the two-cell stage (Fig. 1A). A substantial difference in response to the two doses of GAL4 mRNA in activation of the effector gene was detected by observing ECFP expression in the injected side of embryos at later stages (Fig. 1B and F). This 
unilateral ECFP expression was particularly obvious in enlarged dorsal view images of stage 45 embryos injected with 10 pg of GAL4 mRNA (Fig. 1C-E). Strong RFP expression in somitic muscle driven by the second cassette at stage 45 (Nieuwkoop and Faber, 1967) enabled us to confirm the stable transmission of the transgene through the germline and to select RFP-positive embryos carrying the UAS-target to assay its activation by injected GAL4 (Fig. 1F). ECFP expression was seen in $55 \%$ (21 out of 38) or $95 \%$ (19 out of 20 ) of RFP-positive embryos injected with 1 pg or $10 \mathrm{pg}$ of GAL4 mRNA, respectively. Uninjected RFP-positive embryos showed no visually-detectable ECFP expression (0 out of 10 ). No expression was observed following the injection of any dose of GAL4 into RFP-negative embryos that have no target gene to activate (data not shown).

The F1 animals derived from the transgenic UAS-ECFP founder used for GAL4 RNA injections were raised to maturity and found to contain two different UAS-ECFP inserts in subsequent analyses. DNA from F1 animals was analyzed by genomic Southern blotting, and they showed hybridization patterns which indicate that they carry one of two transgenic loci in their founder (data not shown).

Although ECFP was not detected by fluorescence in the untreated UAS lines, the two F1 populations with distinct Southern patterns were further evaluated for background expression of ECFP by Western blotting, and one was found to have detectable background by this method. Each group of F1 animals was outcrossed and resulting embryos were subjected to Western analysis. A correlation between Southern patterns and background expression of ECFP was thereby revealed; one group showed little 
background while the other group showed a detectable level of background (data not shown). The low background line was used in subsequent experiments.

\section{$R x$ promoter-GFP reporter expression in transgenic $X$. tropicalis lines}

For the GAL4 transactivator line, I chose to target expression to the retina using the promoter from the homeobox gene $R x$ (Mathers et al., 1997). X. laevis $R x$ has been reported to be expressed primarily in the primordia of the retina. A $2.2 \mathrm{~kb} 5$ '-upstream region of the $R x$ gene was obtained by screening a Xenopus tropicalis genomic library with a Xenopus laevis $R x$ cDNA probe. In order to confirm that this promoter sequence could direct expression to the developing retina, I generated transgenic lines with GFP fused downstream of the $2.2 \mathrm{~kb} R x$ promoter (pRx-GFP) and analyzed the F1 embryos for the GFP expression pattern. Strong GFP expression was seen first in the anterior of neural fold stage embryos, and was subsequently localized to the two fields that give rise to the retina (Fig. 2A and B). At later stages, the retina remains the primary site of GFP expression in the pRx-GFP transgenic embryos (Fig. 2C-E). The same promoter sequence that has the upstream region important for $R x$ expression in the retina was utilized to direct the expression of chimeric GAL4 in the activator lines. An advantage of the use of this eye-specific promoter is the relative ease of assaying the activation of effector in vivo by being able to identify embryos showing fluorescence because it is visible externally.

\section{Inducible chimeric GAL4 activates UAS-target in the presence of RU486}

To add the dimension of temporal regulation of target gene expression, I utilized a chimeric transactivator, GALPR, constructed by fusing the GAL4 DNA binding domain 
to a mutated human progesterone receptor ligand binding domain and the VP16 transcriptional activation domain (Fig. 3A) (Wang et al., 1994). Upon binding to synthetic progesterone analogue, RU486, this fusion protein escapes cytoplasmic sequestration, travels to the nucleus, and binds UAS sequences, resulting in efficient ligand-inducible transactivation of target genes in cell culture and transgenic mouse models (Burcin et al., 1998; Wang et al., 1997; Zhao et al., 2001). To establish transgenic $X$. tropicalis lines with retina-specific expression of this chimeric transactivator, I generated frogs bearing the $R x$ promoter driving the GALPR construct (pRx-GALPR). Next, I asked whether the chimeric GAL4 activates the transcription of UAS-target in the progeny of the cross of the two lines (Fig. 3A). The pRx-GALPR transactivator line was crossed with UAS-ECFP effector frogs (Fig. 3A) and the progeny cultured with or without RU486 from stage 22 onward (Fig. 3B). Each of the lines in this cross is hemizygous for a transgenic locus, producing 4 different genotypes: non-transgenic embryos, embryos bearing one transgene (either the effector or transactivator gene), and embryos bearing both effector and transactivator transgenes. Approximately $50 \%$ of the progeny expressed RFP in the somites, indicating that they carry a single locus bearing the UAS-effector construct. Effector gene expression was analyzed either by microscopic examination for ECFP fluorescence in the eye or by western analysis of eye extracts with an EGFP antibody that crossreacts with ECFP (Clontech). The expression of the ECFP in the effector construct is highly localized in the retina and increases in a dose-dependent manner after RU486 treatment in embryos carrying both effector and transactivator genes. This result can be seen in a qualitative way in the series of fluorescent images in Fig. 3B, which illustrates embryos $30 \mathrm{hr}$ and $45 \mathrm{hr}$ after treatment. Table 1 summarizes 
data from embryos carrying the effector transgene treated with $0 \mathrm{nM}, 5 \mathrm{nM}$ and $25 \mathrm{nM}$ RU486, showing that both the fraction of embryos expressing ECFP, and their level of expression, increases with increasing dose of RU486. Western blot analysis of dissected eye tissue (Fig. 3C and D) shows that the level of expression of ECFP in treated embryos depends in a quantitative way on the dose of RU486. ECFP expression was minimal in the absence of RU486, as assayed by ECFP fluorescence or western analysis (Table 1, Fig. 3B-D and data not shown), suggesting that this system is not affected by endogenous frog hormones. I also found that elevated ECFP fluorescence 45 hours postincubation (top panels in Fig. 3B) returned to baseline in some embryos after RU486 was removed from culture media though others still showed significant fluorescence after 2 days of withdrawal (data not shown). While the decay of the reporter gene activity was slow, it appears that the target gene induction is reversible. I conclude that this inducible regulatory system works efficiently in X. tropicalis.

\section{Kinetics of UAS-target induction in response to RU486}

Next, I asked how rapidly the transgene expression is induced upon the application of RU486. To study the kinetics of UAS-ECFP induction in the eye, in situ hybridization analysis was carried out using embryos from the cross of the same pRxGALPR activator and UAS-ECFP effector lines described above. Embryos were cultured from stage 12/13 onward for varying lengths of time in a medium containing 0 or $25 \mathrm{nM}$ RU486, the concentration previously shown to induce maximal ECFP expression. After $2.5,5,7.5$, or $10 \mathrm{hr}$, embryos were fixed and hybridized with a probe specific for the coding sequence of ECFP. The robust induction of ECFP expression was detectable as 
early as $2.5 \mathrm{hr}$ incubation in RU486 (Fig. 2-4). Consistent with the constitutive GFP expression observed in pRx-GFP transgenic animals (Fig. 2-2; also see Fig. 2-4), the induced ECFP expression was confined principally to the developing retina (Fig. 2-4 A, C, D), recapitulating the normal $R x$ gene expression pattern (Fig. 2-3B). Note that approximately a quarter of embryos analyzed showed in situ hybridization staining as each of the activator and effector lines in the cross is hemizygous for a transgenic locus and only embryos carrying both transgenes can express ECFP in the presence of RU486. Embryos cultured in the absence of RU486 did not show ECFP expression (Fig. 2-4A). These results indicate that RU486 can be rapidly assimilated into frog embryos and induce the target gene expression within as little as $2.5 \mathrm{hr}$.

\section{Higher concentration of DMSO solvent does not increase reporter gene expression}

RU486 administration induced the ECFP reporter expression in the presumptive retina (see Fig. 2-4) in a pattern nearly identical to that of endogenous $R x$. Unexpectedly, however unlike endogenous $R x$, the ECFP reporter expression became dispersed as patchy cell clusters throughout the retina later during optic cup development (data not shown; see Fig. 2-5). It was not clear whether this was because of a change in the efficiency of RU486 uptake by embryos or because of a positional effect of the integrated transgenes. To investigate the underlying cause of this problem, I first tested whether the efficiency of penetration of RU486 into the cells inside embryos could be changed by increasing the concentration of dimethyl sulfoxide (DMSO), the solvent used to dissolve water-insoluble RU486. DMSO was chosen because it also potentiates the permeability of skin barrier to applied substances. As in previous studies of teratogenic effects of 
DMSO using X. laevis embryos (Dresser et al., 1992; Rayburn et al., 1991), I found that culture media containing DMSO at a concentration under $1 \%(\mathrm{v} / \mathrm{v})$ did not significantly interfere with normal development of $X$. tropicalis embryos. Developmental abnormalities including ocular (arrow in Fig. 2-5A) and body axis (arrows in Fig. 2-5B) phenotypes were greatly increased by exposure to 1.5\% DMSO (Fig. 2-5C) as previously reported (Dresser et al., 1992). However, the RU486-mediated ECFP reporter expression did not seem to be increased or altered significantly even with the highest concentration of DMSO I tested. Embryos cultured in $0.5-1.5 \%$ DMSO all showed a similar dispersed punctate pattern within the st 38 or 42 retina (Fig. 2-5E-F, H-I, and K-L; data not shown), which was similar to the previously observed expression pattern (data not shown; see D and $G$ ) in samples where only trace amounts of DMSO shown as 0\% in Fig. 2-5 were present (resulting from the addition of RU486, which was dissolved in DMSO as a solvent). These results suggest that higher concentration of DMSO solvent did not alter the reporter expression pattern. It is not clear, however, because of the response being assayed here, whether the efficiency of RU486 delivery was increased by the different DMSO doses I tested.

\section{Patchy reporter activation does not result from nonuniform GAL4 activator expression}

Next, I asked whether the possible positional effect of the GAL4 transgene contributes to the punctate ECFP expression of the reporter. To test the correlation of the patchy ECFP activation with the expression of GALPR, in situ hybridization analysis was performed. I compared the expression pattern of the GALPR transcript in pRx-GALPR 
transgenic embryos with the expression of the endogenous $R x$ and GFP from pRx-GFP animals. As expected, the GALPR RNA was primarily localized to the primordia of the retina throughout the stages analyzed, recapitulating $R x$ expression. Even at later stages, when the ECFP effector expression became patchy, the GALPR expression was not patchy but, rather uniform within the retina, almost identical to that of the endogenous $R x$ or GFP directed by pRx-GFP (compare Fig. 2-6F with $\mathrm{G}$ and $\mathrm{H}$; J with $\mathrm{K}$ and $\mathrm{L}$ ). Therefore I concluded that the patchy ECFP effector expression did not result from patchy GALPR mRNA expression, although it is still possible that that activation of GALPR protein by RU486 occurs nonuniformly (see Discussion).

In this Chapter, I have shown that the hormone-inducible GAL4 can efficiently transactivate the UAS-reporter expression in RU486- and tissue-specific fashion. In the following Chapter, I tested the utility of this inducible gene targeting system for the study of gene function during Xenopus development.

\section{Discussion}

In this study, I have evaluated the utility of a GAL4/UAS system in Xenopus tropicalis. In the stable transgenic activator and effector lines I established, transgenic GAL4 efficiently activates the transcription of a reporter gene in the cells where it is expressed. Development of a GAL4/UAS method for targeted gene expression in the $X$. tropicalis system is desirable for several reasons. First, $X$. tropicalis' significantly shorter generation time of 3-5 months allows rapid production and expansion of stable GAL4 and UAS transgenic lines at a relatively low cost. Second, the efficiency of in vivo 
promoter analysis in transgenic Xenopus (Offield et al., 2000) is resulting in an accumulation of defined promoters. These promoters, when combined with GAL4, provide very useful tools for expressing combinations of effector constructs in analysis of a wide variety of developmental processes. Third, the GAL4/UAS system has many potential applications for gene function studies (Phelps and Brand, 1998). For example, this method may be used to produce loss-of-function phenotypes by driving the expression of a gene variant that will antagonize the endogenous gene product. This is highly desirable because currently there is no technique for targeted gene disruption in Xenopus.

In addition to showing the utility of a GAL4/UAS system in Xenopus for spatial regulation of transgenic constructs, I also have used the construct to achieve the potential for temporal control of gene expression. A ligand-inducible regulatory system based on a mutated progesterone receptor ligand binding domain has been shown to work in adult mouse cells and organs (Wang et al., 1997; Zhao et al., 2001). I show here that this inducible system can be used to study early embryonic development. I demonstrated that this system regulates gene expression in vivo in $X$. tropicalis embryos in response to RU486. I observed that embryos cultured in $25 \mathrm{nM}$ of RU486 showed a strong induction of the target gene with no observable developmental defects (data not shown). I should mention that the induction is possible at concentrations that are significantly lower than the micromolar range required to antagonize progesterone (Wang et al., 1994). Therefore, RU486 treatment is unlikely to affect this endogenous hormone system.

While rapid kinetics of induction is a desirable aspect of any inducible system, I observed a delay in target gene induction, with ECFP fluorescence first detected 17 hours 
postincubation in RU486. However, it has been reported that detectable protein fluorescence lags behind the onset of UAS-GFP gene transcription, possibly due to posttranslational modification and folding (Brand, 1995; Phelps and Brand, 1998). Consistent with this, maturation of EGFP (Clontech) takes about 8-9 hours in Xenopus when tested by microinjection of EGFP mRNA into 2-4 cell embryos (A. Zaraisky, personal communication). Considering that the concentration of mRNA transcribed from the transgene is, in general, lower than that used in microinjection, the time lag for ECFP accumulation to detectable levels would be expected to be considerably longer than 8-9 hours. I might expect that induction of other genes used in applications of this system would be substantially faster than that of ECFP shown here. The transcriptional activation of the effector transgene was indeed detected within $2.5 \mathrm{hr}$ after RU486 application. Thus, in contrast to the slow decay of the reporter activity after RU486 removal, the induction of the effector is very rapid.

The early ECFP expression which was uniformly distributed throughout the $R x$ expression domain in the optic vesicle became diffuse and patchy later during optic cup stages. Several alternative reasons could explain this dispersed reporter expression. Higher concentration of DMSO solvent did not increase the ECFP expression. It is presently unknown whether this is because RU486 uptake by cells reached saturating levels even with a low concentration of DMSO or because the highest dose I tested was simply not sufficient to increase the solubility and permeability of RU486. However, the latter explanation is unlikely, as RU486 seems to be efficiently solubilized at the concentrations I tested (Reilly et al., 1999; Sakamoto et al., 2001). In addition, RU486 
combined with a low concentration of DMSO efficiently activated the reporter gene through a similar inducible GAL4 system in Drosophila (Osterwalder et al., 2001).

I confirmed that the diffuse, punctate activation of the UAS-ECFP reporter did not result from nonuniform GALPR expression, although it is still possible that the activation of GALPR protein by RU486 could take place unevenly within the tissue where the GALPR RNA is transcribed. Another possibility is that as with Drosophila, where the GAL4 system has been extensively applied, there could be simple variations of the UAS effector in the response to activated GALPR protein among cells within the same tissue. Mechanisms explaining these variations would include a potential effect of the UAS-ECFP integration site on its transcription, specially at stages when the patchy expression was observed. As I have observed variations in ECFP expression between two different integrations containing the same UAS-ECFP construct, it might be necessary to screen through effector lines to find a line with a uniform expression pattern (Hartley et al., 2001). Another potential solution for the proposed positional effect, in general, would be the addition of insulator sequences conferring integration site-independent transgene expression (Burcin et al., 1999; Wang et al., 1997).

The patchy ECFP reporter expression could also be explained by GALPR transcription localized in the ciliary marginal zone (CMZ). At stages when ECFP expression is detected in several cells dispersed throughout the neural retina, GALPR or $R x$ RNA normally becomes restricted to the cells of the retinal CMZ (see Fig. 2-6F-H, JK), which are the multipotent mitotic retinal cells (Mathers et al., 1997; Wetts et al., 1989). These $R x$-expressing cells become gradually postmitotic and differentiated as they leave the peripheral CMZ. As they are incorporated into the central retina, some of these 
cells lose $R x$ expression (Perron et al., 1998). Cell linage analysis has revealed that labeled descendants from cells in CMZ differentiated into all of the major retinal cell types (Wetts et al., 1989). There is a potential time delay between the accumulation of GAL4 protein and its transactivation of an effector. Thus, when the effector ECFP expression is detectable, the cells, which were expressing GALPR RNA in the CMZ, already have left the $\mathrm{CMZ}$ and migrated into the central retina. Perron et al (1998) have reported that $X$. laevis $R x$ was faintly expressed in a dispersed subset of cells of the inner nuclear layer and in the photoreceptor layer as well as in the CMZ although I was unable to detect this expression in $X$. tropicalis central retina at similar stage (Fig. 2-6). This is unlikely to explain the patchy reporter expression because $(i)$ the cells expressing ECFP were found in all three layers of the st 40/41 retina, (ii) the ECFP expression was detected in clusters of cells with a variable size dispersed throughout the st $37 / 38$ retina.

The patchy distribution of ECFP expression also could be explained by 'desensitization' of the treated cells to RU486 (Qi et al., 1990). This seems unlikely as similar RU486-mediated GAL4 expression systems in other organisms including mouse and Drosophila did not show desensitization from the continuous presence of ligand or induced activator (Burcin et al., 1999; Roman et al., 2001). For example, flies administered RU486 for a full $24 \mathrm{hr}$ displayed as robust reporter activity as flies treated for only $1 \mathrm{hr}$, suggesting RU486-responsive cell were not desensitized, at least within 24 hr. As Xenopus embryos showed punctate reporter expression after 30 hours of continual incubation it would be valuable to follow the expression profile of the effector after a short period of RU486 treatment. 
For the applications usually requiring robust transgene expression such as the gene perturbation assays mentioned earlier, further modulation of induction rapidity and expression level of the effector transgene could be achieved in several ways. First, one can make transgenic lines with very high numbers of inserts (e.g. 30-40 copies) in the Xenopus system, either for the effector or transactivator, or both. One could also increase the number of GAL4 binding sites upstream of the target gene (Brand and Perrimon, 1993; Rorth, 1996). In addition, one could insert 5' or 3' UTR sequences that would stabilize the transcript from the transgene (Rupp et al., 1994; Turner and Weintraub, 1994). Functional inhibition of some gene products, such as homeodomain transcription factors including Pax6 and Otx homologs, Crx and SpOtx2, may not require very high levels of overexpression of dominant-negative mutants and therefore the expression level of mutant effector transgene producing the phenotype would be readily reached by the method described here. These genes have been shown to be repressed effectively by dominant-inhibitory variants at concentrations lower than those of wild-type products (Furukawa et al., 1997; Li et al., 1999; Singh et al., 1998).

While culturing embryos with RU486 at stage 22 resulted in 14-fold induction of target gene expression, I also treated with RU486 at stage 41 onward and observed comparable activation of ECFP expression (data not shown). This suggests that this system can overcome a major limitation of RNA expression studies in Xenopus, that the instability of RNA prevents analysis of its effects on later stages, and may be used to express effector transgenes at all stages of development, facilitating analysis of genes that function both in early embryogenesis and later developmental processes such as organogenesis. 


\section{References}

Amaya, E., Offield, M. F. and Grainger, R. M. (1998). Frog genetics: Xenopus tropicalis jumps into the future. Trends in Genetics 14, 253-5.

Brand, A. (1995). GFP in Drosophila. Trends Genet 11, 324-5.

Brand, A. H. and Perrimon, N. (1993). Targeted gene expression as a means of altering cell fates and generating dominant phenotypes. Development 118, 401-15.

Bronchain, O. J., Hartley, K. O. and Amaya, E. (1999). A gene trap approach in Xenopus. Current Biology 9, 1195-1198.

Burcin, M. M., BW, O. M. and Tsai, S. Y. (1998). A regulatory system for target gene expression. Front Biosci 3, c1-7.

Burcin, M. M., Schiedner, G., Kochanek, S., Tsai, S. Y. and O'Malley, B. W. (1999). Adenovirus-mediated regulable target gene expression in vivo. Proceedings of the National Academy of Sciences of the United States of America 96, 355-60.

Dresser, T. H., Rivera, E. R., Hoffmann, F. J. and Finch, R. A. (1992). Teratogenic assessment of four solvents using the Frog Embryo Teratogenesis Assay--Xenopus (FETAX). J Appl Toxicol 12, 49-56.

Furukawa, T., Morrow, E. M. and Cepko, C. L. (1997). Crx, a novel otx-like homeobox gene, shows photoreceptor-specific expression and regulates photoreceptor differentiation. Cell 91, 531-41.

Harland, R. M. (1991). In situ hybridization: an improved whole-mount method for Xenopus embryos. Methods in Cell Biology 36, 685-695. 
Hartley, K. O., Hardcastle, Z., Friday, R. V., Amaya, E. and Papalopulu, N. (2001). Transgenic Xenopus embryos reveal that anterior neural development requires continued suppression of BMP signaling after gastrulation. Developmental Biology 238, 168-84. Kroll, K. L. and Amaya, E. (1996). Transgenic Xenopus embryos from sperm nuclear transplantations reveal FGF signaling requirements during gastrulation. Development 122, 3173-83.

Li, X., Wikramanayake, A. H. and Klein, W. H. (1999). Requirement of SpOtx in cell fate decisions in the sea urchin embryo and possible role as a mediator of beta-catenin signaling. Developmental Biology 212, 425-39.

Mathers, P. H., Grinberg, A., Mahon, K. A. and Jamrich, M. (1997). The Rx homeobox gene is essential for vertebrate eye development. Nature 387, 603-7. Nieuwkoop, P. D. and Faber, J. (1967). Normal table of Xenopus laevis. Amsterdam: North Holland Publishing.

Offield, M. F., Hirsch, N. and Grainger, R. M. (2000). The development of Xenopus tropicalis transgenic lines and their use in studying lens developmental timing in living embryos. Development 127, 1789-97.

Osterwalder, T., Yoon, K. S., White, B. H. and Keshishian, H. (2001). A conditional tissue-specific transgene expression system using inducible GAL4. Proceedings of the National Academy of Sciences of the United States of America 98, 12596-601.

Perron, M., Kanekar, S., Vetter, M. L. and Harris, W. A. (1998). The genetic sequence of retinal development in the ciliary margin of the Xenopus eye. Developmental Biology 199, 185-200. 
Phelps, C. B. and Brand, A. H. (1998). Ectopic gene expression in Drosophila using GAL4 system. Methods 14, 367-79.

Pierson, T. M., Wang, Y., DeMayo, F. J., Matzuk, M. M., Tsai, S. Y. and Omalley, B. W. (2000). Regulable expression of inhibin A in wild-type and inhibin alpha null mice. Molecular Endocrinology 14, 1075-85.

Qi, M., Stasenko, L. J. and DeFranco, D. B. (1990). Recycling and desensitization of glucocorticoid receptors in v-mos transformed cells depend on the ability of nuclear receptors to modulate gene expression. Molecular Endocrinology 4, 455-64.

Rayburn, J. R., DeYoung, D. J., Bantle, J. A., Fort, D. J. and McNew, R. (1991). Altered developmental toxicity caused by three carrier solvents. J Appl Toxicol 11, 25360.

Reilly, P. E., Gomi, R. J. and Mason, S. R. (1999). Mechanism-based inhibition of rat liver microsomal diazepam C3-hydroxylase by mifepristone associated with loss of spectrally detectable cytochrome P450. Chem Biol Interact 118, 39-49.

Roman, G., Endo, K., Zong, L. and Davis, R. L. (2001). P[Switch], a system for spatial and temporal control of gene expression in Drosophila melanogaster. Proceedings of the National Academy of Sciences of the United States of America 98, 12602-7.

Rorth, P. (1996). A modular misexpression screen in Drosophila detecting tissuespecific phenotypes. Proceedings of the National Academy of Sciences of the United States of America 93, 12418-22.

Rupp, R. A. W., Snider, L. and Weintraub, H. (1994). Xenopus embryos regulate the nuclear localization of XMyoD. Genes and Development 8, 1311-1323. 
Sakamoto, H., Ukena, K. and Tsutsui, K. (2001). Effects of progesterone synthesized de novo in the developing Purkinje cell on its dendritic growth and synaptogenesis. $J$ Neurosci 21, 6221-32.

Singh, S., Tang, H. K., Lee, J. Y. and Saunders, G. F. (1998). Truncation mutations in the transactivation region of PAX6 result in dominant-negative mutants. $J$ Biol Chem 273, 21531-41.

Sive, H. L., Grainger, R. M. and Harland, R. M. (2000). Early development of Xenopus laevis. A laboratory manual. New York: Cold Spring Harbor Laboratory Press. Turner, D. L. and Weintraub, H. (1994). Expression of achaete-scute homolog 3 in Xenopus embryos converts ectodermal cells to a neural fate. Genes and Development 8 , 1434-1447.

Vegeto, E., Allan, G. F., Schrader, W. T., Tsai, M. J., McDonnell, D. P. and O'Malley, B. W. (1992). The mechanism of RU486 antagonism is dependent on the conformation of the carboxy-terminal tail of the human progesterone receptor. Cell 69, 703-13.

Wang, X. J., Liefer, K. M., Tsai, S., O'Malley, B. W. and Roop, D. R. (1999).

Development of gene-switch transgenic mice that inducibly express transforming growth factor betal in the epidermis. Proceedings of the National Academy of Sciences of the United States of America 96, 8483-8.

Wang, Y., DeMayo, F. J., Tsai, S. Y. and O'Malley, B. W. (1997). Ligand-inducible and liver-specific target gene expression in transgenic mice. Nat Biotechnol 15, 239-43. 
Wang, Y., O'Malley, B. W., Jr., Tsai, S. Y. and O'Malley, B. W. (1994). A regulatory system for use in gene transfer. Proceedings of the National Academy of Sciences of the United States of America 91, 8180-4.

Wetts, R., Serbedzija, G. N. and Fraser, S. E. (1989). Cell lineage analysis reveals multipotent precursors in the ciliary margin of the frog retina. Developmental Biology 136, 254-63.

Zhao, B., Chua, S. S., Burcin, M. M., Reynolds, S. D., Stripp, B. R., Edwards, R. A., Finegold, M. J., Tsai, S. Y. and DeMayo, F. J. (2001). Phenotypic consequences of lung-specific inducible expression of FGF-3. Proceedings of the National Academy of Sciences of the United States of America 98, 5898-903. 
Figure 2-1. Injected GAL4 RNA activates the expression of transgenic UAS effector gene. (A) Experimental outline. Injection of GAL4 RNA unilaterally into 2-cell stage UAS-ECFP F1 transgenic embryos. GAL4 protein (blue ovals) binds to UAS sequences, activating transcription of the ECFP reporter (blue arrow). The UAS-ECFP construct carries a second reporter cassette, pCAR-RFP, to drive RFP expression in somitic muscle (red arrow), to delineate transgenic embryos. (B) Dorsal view of stage 19 embryos injected with indicated dose of GAL4. uninj: uninjected non-transgenic sibling control. RFP+ siblings which did not receive GAL4 RNA were likewise ECFP- (data not shown). Shown in a higher magnification are bright field (C), GFP (D), and RFP (E) images of dorsal view of embryos injected with $10 \mathrm{pg}$ in (F). Strong ECFP expression is seen on the injected side of embryos. (F) Lateral view of injected sides of stage 45 embryos. Note that some RFP expression remains discernable with the GFP filter used here (D, F). 


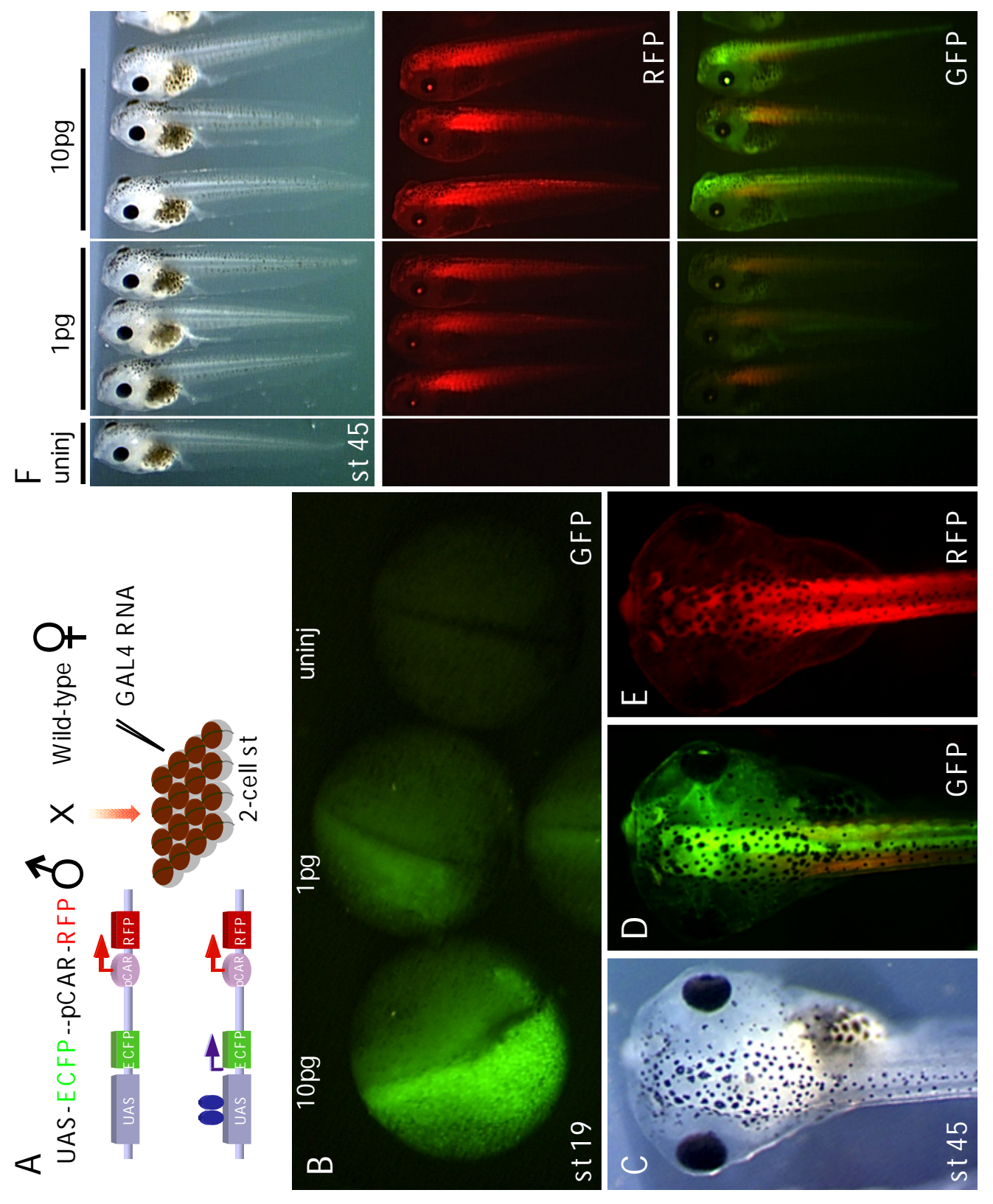


Figure 2-2. $R x$ promoter directs GFP reporter expression to the eye in transgenic $X$. tropicalis embryos. A $2.2 \mathrm{~kb}$ genomic fragment from the $R x$ gene was fused to GFP (pRx-GFP) and used to establish stable $X$. tropicalis transgenic reporter lines. (A-E) Fluorescent images of pRx-GFP transgenic embryos at indicated stages. The identical promoter sequence was used to drive expression of the chimeric GAL4 transactivator in pRx-GALPR transgenic lines (see Figure 3). 


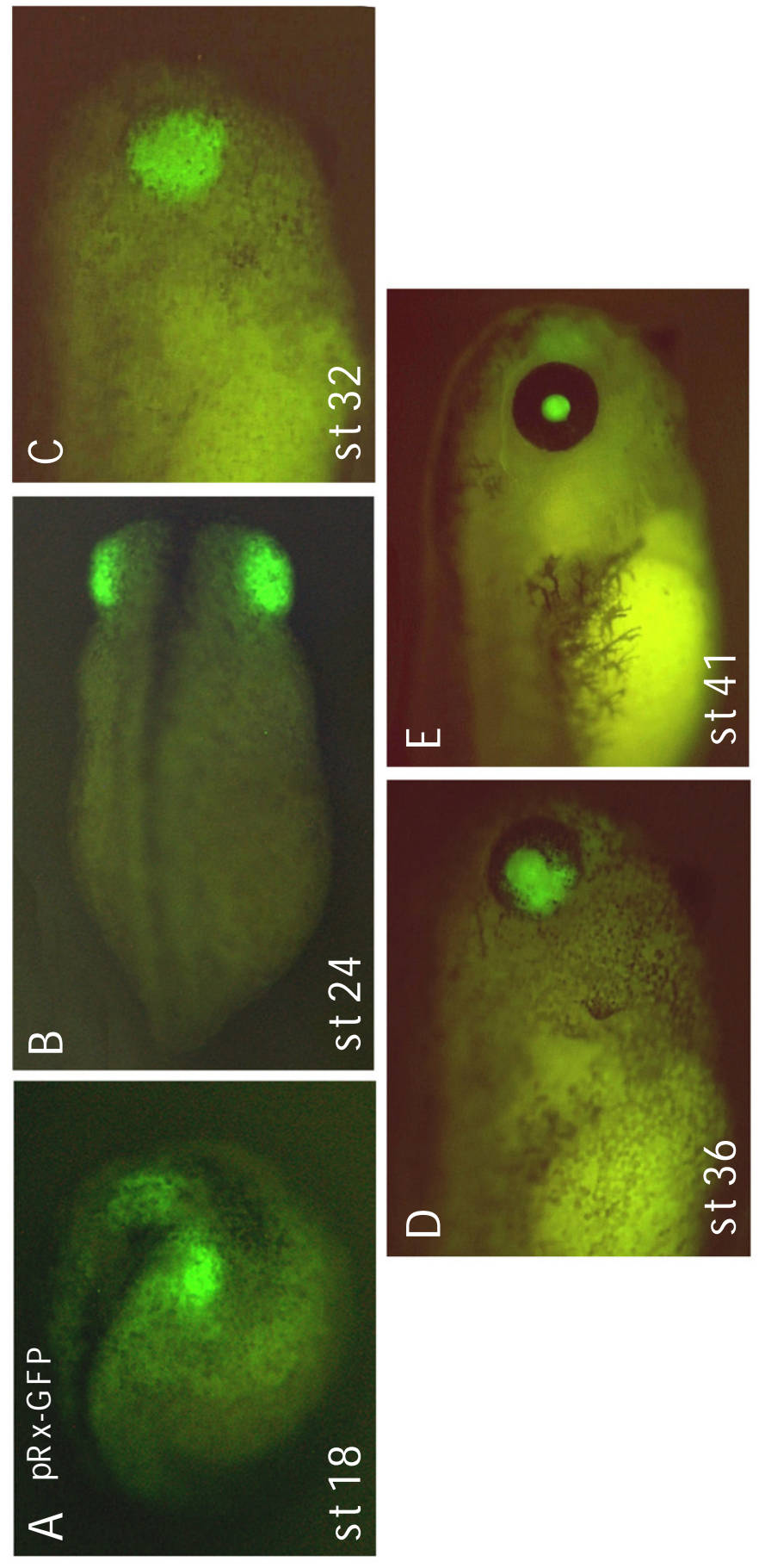


Figure 2-3. Inducible GAL4 transgenic construct activates UAS-target gene in response to RU486. (A) A UAS-ECFP line was crossed to a pRx-GALPR line, in which the $R x$ promoter drives a chimeric transactivator gene (GAL4 DNA-binding domain, fused to a Herpes virus VP16 transcriptional activation domain, in turn fused to a modified progesterone receptor ligand-binding domain). In the double-transgenic progeny, transcription of the UAS-ECFP reporter cassette is activated only when the synthetic ligand RU486 is present, relieving the cytoplasmic sequestration of the GALPR transactivator protein. (B) Representative embryos from this cross, treated with 0,5 , or $25 \mathrm{nM}$ RU486 from stage 22 onwards for 30 (bottom panels) or 45 (top panels) hours. ECFP expression was observed only in RFP-positive embryos. (C) Western blot analysis of eye extract from embryos shown in (B) using EGFP antibody and anti-actin antibody. A pool of 30 RFP-positive or RFP-negative embryos at each dose of RU486 was assayed in each lane. (D) Western blot band intensities are quantified by densitometry, and relative levels of ECFP expression are calculated by normalizing to actin levels. 

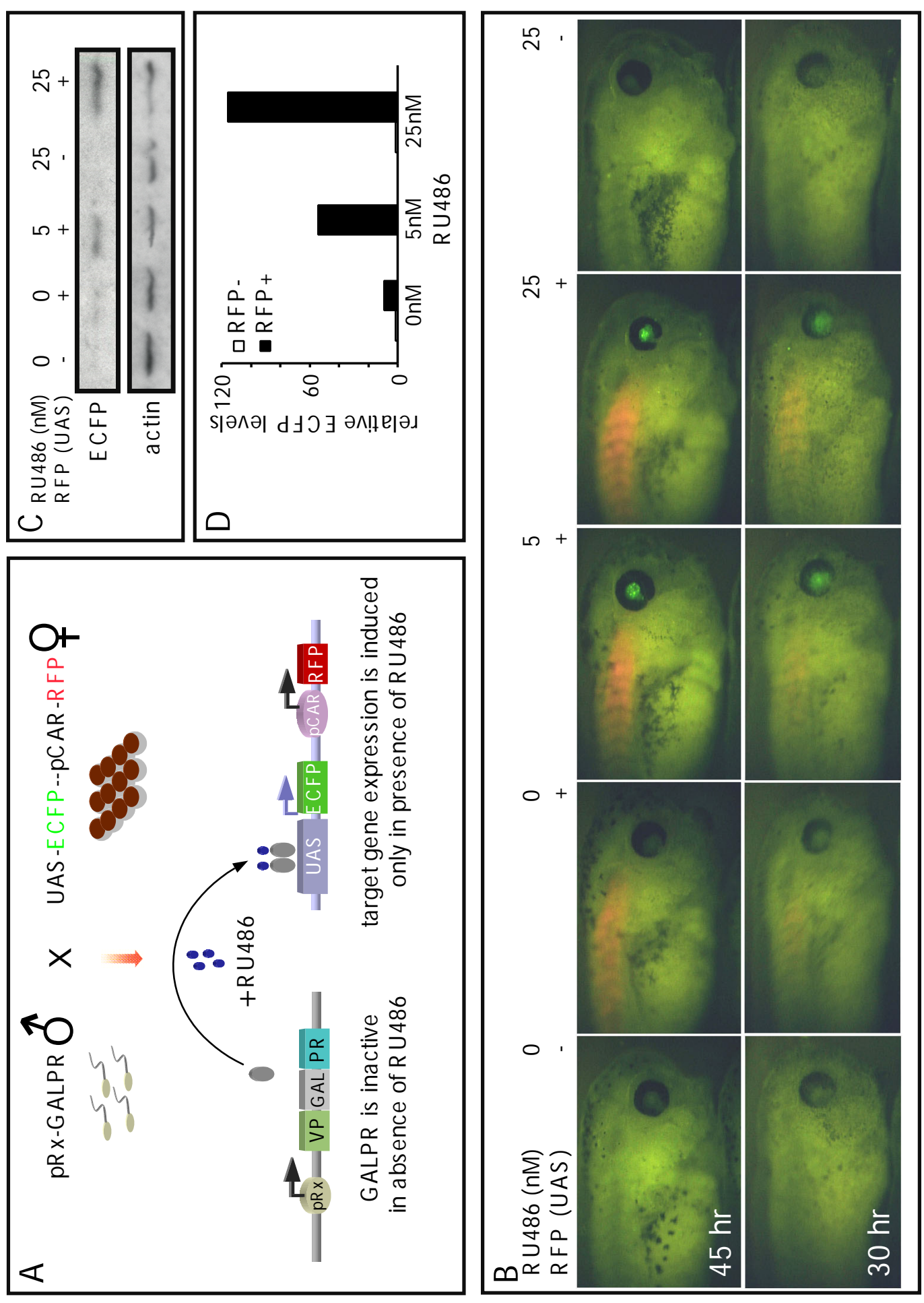
Table 2-1. Target gene (ECFP) expression in the progeny of the cross of pRx-GALPR and UAS-ECFP animals

$\mathrm{ECFP}++\quad \mathrm{ECFP}+\quad \mathrm{ECFP}-$

$\begin{array}{cccc}0 \text { nM RU486 } & 0 & 0 & 50 \\ 5 \text { nM RU486 } & 3 & 10 & 37 \\ 25 \text { nM RU486 } & 11 & 8 & 31\end{array}$

Embryos from the cross of the pRx-GALPR activator line with the UAS-ECFP effector line were incubated with RU486 for 30 hours and assayed for ECFP expression. Each of 50 embryos carrying the effector gene (i.e., RFP-positive) cultured in 0 (solvent control), 5 , or $25 \mathrm{nM}$ of RU486, respectively were scored as strongly ECFP-positive (++), weakly ECFP-positive $(+)$ or -negative (-). At the higher dose of RU486, the fraction of embryos expressing ECFP approaches the expected maximum level of $50 \%$, i.e. the fraction of embryos expected to carry both activator and effector transgenes. Effector-negative embryos (i.e. RFP-negative) were also analyzed for ECFP expression in the same way and showed no detectable ECFP expression. 
Figure 2-4. Rapid induction of RU486-mediated effector expression. (A) Embryos from the cross described in Fig. 2-3 were cultured with RU486 from stage 12/13 onward for the indicated times, and then fixed and analyzed for the expression of ECFP by in situ hybridization using an ECFP probe. In the table above A, the induction of ECFP expression is indicated as positive $(+)$ and negative $(-)$. Note that even in the presence of RU486 (25 nM), only about a quarter of total embryos at each time point exhibit the staining. Solvent-treated $(0 \mathrm{nM})$ embryos do not show ECFP expression (i.e. ECFP induction-negative). (B) A transverse section of a wild-type embryo at stage similar to C, hybridized with an $R x$ probe. (C, D) Transverse sections of $7.5 \mathrm{hr}$ and $10 \mathrm{hr}$ postincubated ECFP-positive embryos in the panels above, respectively. Note that staining is confined to the presumptive retina only and is not seen in the overlying lens ectoderm (arrows in D). 


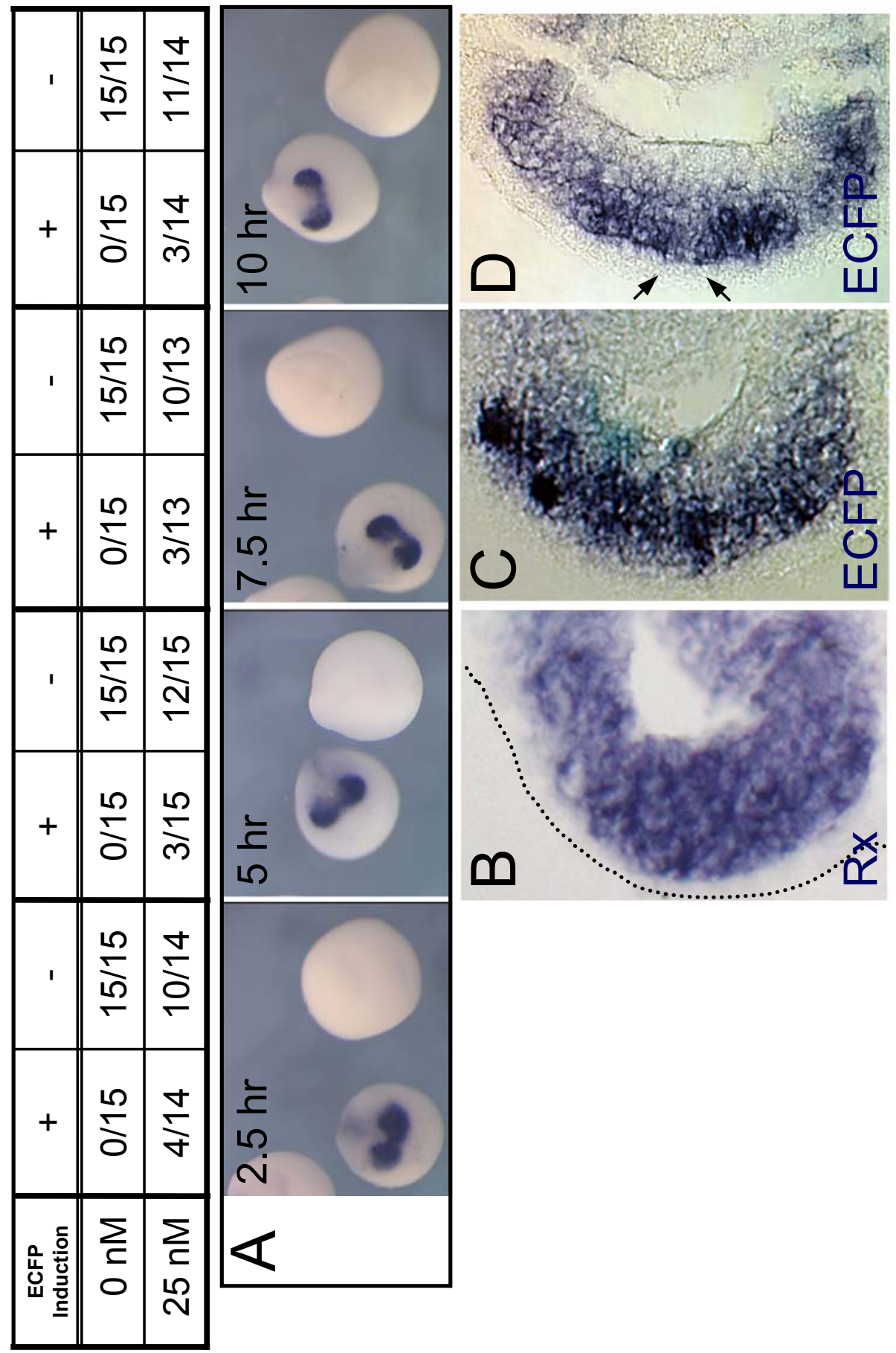


Figure 2-5. ECFP reporter induction by RU486 combined with different doses of DMSO solvent. UAS+ (i.e. RFP-positive) embryos from the cross described in Fig. 2-3 were cultured in a medium containing $25 \mathrm{nM}$ RU486 and $0,0.5,1.0$, or $1.5 \%(\mathrm{v} / \mathrm{v})$ DMSO from st $12(\mathrm{M})$ or st 22 (all other panels except M) onward. (A, B) embryos exposed to $1.5 \%$ of DMSO show developmental defects including malformation of eye (arrow in A) and body axis (arrows in B). (C) A table showing the numbers of embryos displaying abnormalities at the indicated concentration of DMSO. Note that the frequency of appearance of malformations increases with increasing concentrations. * indicates very weakly affected embryos. (D-F) Stage 42 embryos showing ECFP expression in the eye (arrowheads) at DMSO concentration of $0,1.0$, and $1.5 \%$, respectively. (G-I) Cryosections of embryos shown in D, E, and F, respectively. Punctate ECFP expression is detected in a few cells dispersed throughout the retinal layers (arrows). Note that higher concentration of DMSO fails to increase the number of cells expressing ECFP $(\mathrm{H}$, I). (J-N) Expression of ECFP RNA in st 38 embryos exposed to DMSO at the indicated concentrations, as detected by whole-mount in situ hybridization with an ECFP probe. (J) A pRx-GFP embryo showing strong constitutive GFP expression in the eye. (K-M) Induced ECFP expression shows diffuse, patchy pattern throughout the retina (arrows). RU486 treatment at early stage (stage 12) did not significantly increase the number of clusters of expression (M), nor did higher DMSO concentration (K). (N) A control embryo cultured in the absence of RU486 displaying no ECFP expression. 


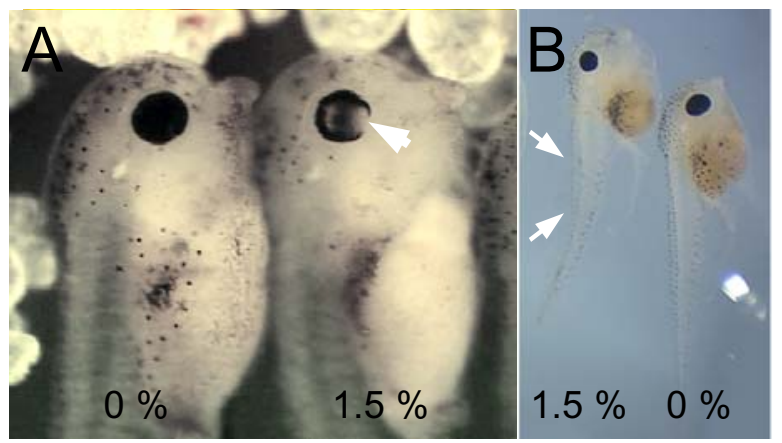

\begin{tabular}{|l|c|c|c|c|}
\hline DMSO conc. & $0 \%$ & $0.5 \%$ & $1.0 \%$ & $1.5 \%$ \\
\hline Abnormal body axis & $0 / 11$ & $1 / 13$ & $* 4 / 11$ & $11 / 11$ \\
\hline Defects in eyes & $0 / 11$ & $0 / 13$ & $0 / 11$ & $2 / 11$ \\
\hline
\end{tabular}

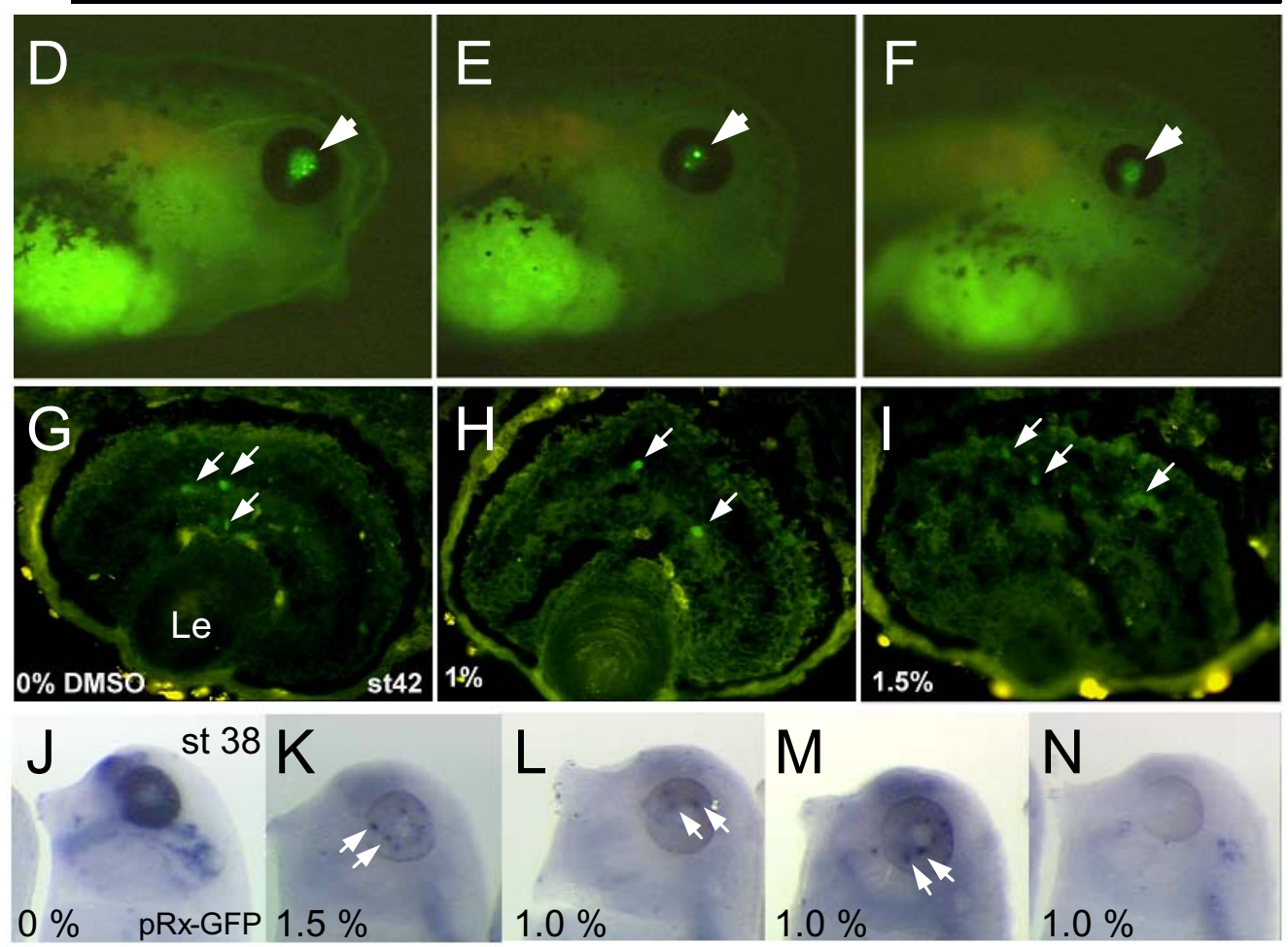


Figure 2-6. Comparison of transcription of $\mathrm{pRx}-\mathrm{GALPR}, \mathrm{pRx}-\mathrm{GFP}$, and $R x$. (A-F, I-J) Expression of GALPR RNA in embryos from pRx-GALPR outcross as detected in whole-mount (A-D) or sections (E, F, I, J) after in situ hybridization using a GAL4 probe at the indicated stages. Transcription of $R x(\mathrm{G}, \mathrm{K})$ and GFP $(\mathrm{H}, \mathrm{L})$ in pRx-GFP embryos as detected in sections after whole-mount in situ hybridization using GFP and $R x$ probes, respectively, at the indicated stages. During optic cup formation, GALPR expression is uniformly distributed throughout the inner neuroblastic layer of the retina (E, I). Expression of GALPR in the retinal CMZ of the optic cup (F, J) is similar to that of $R x$ $(\mathrm{G}, \mathrm{K})$ and GFP $(\mathrm{H}, \mathrm{L})$ in pRx-GFP embryos at corresponding stages. Note that no GALPR expression was detected in the cells of developing lens (arrows in D and E; F, I, $\mathrm{J})$. 


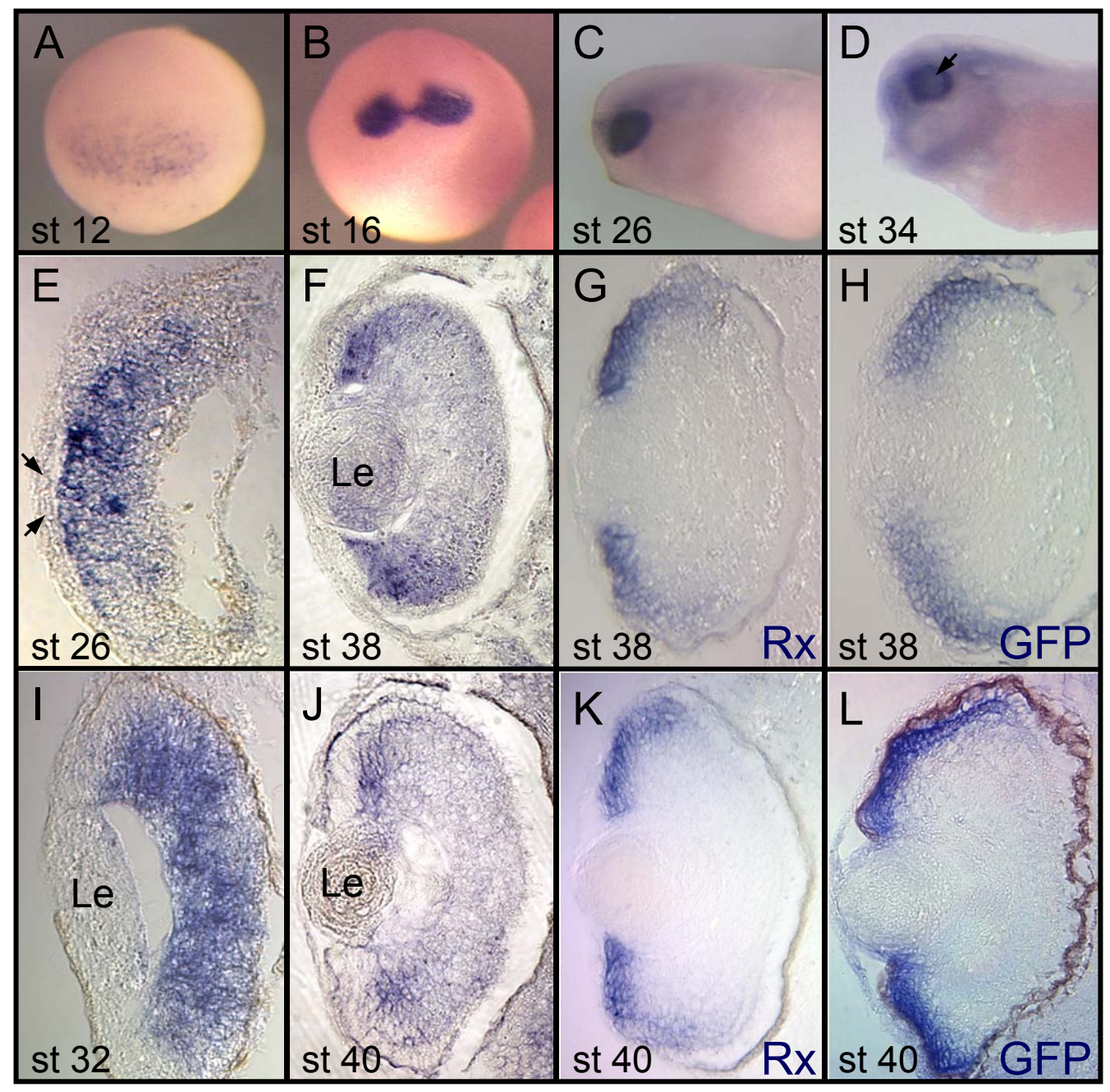




\section{Chapter 3:}

Functional inactivation of $O t x 2$ in the developing retina: application of an inducible gene expression system to study tissue-specific gene function 


\section{Abstract}

Otx2 is a member of a highly conserved family of homeodomain-containing transcription factors that function in early head development. Knockouts of the mouse Otx2 are lacking head structures, including eyes, but it is impossible to discern whether the latter is due to a direct or indirect effect of the mutation. The interpretation of the phenotype is further complicated by its expression pattern and possible roles in both retina and lens ectoderm. To properly test the autonomous effect of the Otx2 mutation on the eye, transcriptional activity of Otx2 must be blocked specifically in retina, but not lens ectoderm, or vice versa.

This question was evaluated initially by primary transgenic assays that repress Otx2 activity in retina by targeting expression of dominant negative forms of Otx2 to the developing retina using a retina-specific promoter. However, analysis of phenotypic consequences were complicated by background abnormalities associated with the transgenic procedure based on the nuclear transfer technique, making it difficult to raise lines carrying the construct. These problems can be circumvented by a GAL4/UAS system that permits establishment of stable transgenic lines.

The inducible GAL4/UAS system was utilized to determine Otx2 function during eye development by targeting expression of dominant negative constructs to retinal tissue. This experiment also tested the utility of the inducible GAL4/UAS system for controlling transgene expression in both time and space for the study of gene function during Xenopus development. A transactivator line driving ligand-inducible GAL4 specifically in the retina was crossed to a UAS-Otx2-EnR line which carries a UAS- 
driven chimeric construct, Otx2-EnR, the Otx2 homeodomain fused to the Drosophila engrailed repressor domain, designed to antagonize the endogenous Otx2 gene product. Embryos inheriting both the GAL4 activator and Otx2-EnR effector displayed the transgene expression within the target tissue and the consequent eye phenotype in response to RU486. In wild-type embryos, the optic vesicle elongates dorsally after its initial outpocketing from the anterior neural tube, positioning the presumptive RPE adjacent to the invaginating distal presumptive neural retina. These RPE cells become the thin proximal wall of the eye cup later. In embryos expressing Otx2-EnR, these morphogenetic movements of the optic vesicle were profoundly disrupted. The pigmented epithelial cells formed a thick layer thus remained dorsally and the separation of the double-layered eye cup from the neural tube failed, leaving eyes fused to the forebrain through a continuous lumen. Later in development, further ventral movement of retinal cells for the completion of the eye-cup formation was also severely impaired, resulting in a failure to close the choroid fissure and a partial or complete loss of the ventral RPE. In addition, the failure of proper retinal layering was also observed. Abnormalities in lens formation indicate that the Otx2 activity in the retina is also required for the determination and differentiation of the lens.

Based on these observations, I propose a model that Otx2 is required for vertebrate eye development, and particularly for the morphogenesis and development of both retina and the lens. These results demonstrate the applicability of the inducible binary system for controlled gene function studies in $X$. tropicalis. 


\section{Introduction}

Otx2 is one of the vertebrate homologs of the Drosophila orthodenticle gene (Finkelstein et al., 1990). This gene encodes a homeodomain-containing transcription factor (Simeone et al., 1993). The otd-deficient Drosophila embryos lack the most anterior portion of the head (Finkelstein et al., 1990). In addition, Otx2 knockout mice do not form a recognizable rostral brain (Acampora et al., 1995; Ang et al., 1996; Rhinn et al., 1998). Thus, this gene has been considered as essential for the specification and regionalization of the brain (reviewed in Acampora et al., 2001; Simeone et al., 2002). Consistent with these loss-of-function phenotypes, it is expressed in the anteriormost part of the neuroectoderm that gives rise to the forebrain and the midbrain (Mallamaci et al., 1996; Simeone et al., 1993). From neurula stages onward, this gene is expressed in anterior neural and placodal ectoderm including the optic vesicle, the primordia of the retina and lens placode as well as nasal placode (Blitz and Cho, 1995; Pannese et al., 1995; Simeone et al., 1993; Zygar et al., 1998), suggesting that it might be involved in the development of sensory organs including eye. Expression analyses of Otx2 in different species including Xenopus (Baas et al., 2000; Blitz and Cho, 1995; Bovolenta et al., 1997; Kablar et al., 1996; Martinez-Morales et al., 2001; Nishida et al., 2003; Pannese

et al., 1995; Perron et al., 1998; Viczian et al., 2003; Zygar et al., 1998) suggest that Otx2 might be very generally involved in the vertebrate eye development.

Although, Otx2 homologs are all expressed in the developing retina prior to retinal differentiation, comparison of the Otx2 expression patterns of Xenopus and mice during ocular development has revealed that there are two major differences between 
these species. First, Xenopus Otx2 is expressed uniformly throughout the optic vesicle (Kablar et al., 1996; Zygar et. al., 1998; in this study) whereas the mouse Otx2 (and Otx1) expression is restricted to the dorsal half of the optic vesicle, the presumptive RPE (Martinez-Morales et al., 2001). As the optic cup forms, mouse Otx2 remains in the proximal layer of the retina, the future RPE, and is maintained in the pigmented epithelium during subsequent stages of development (Baas et al., 2000; Martinez-Morales et al., 2001; Nishida et al., 2003) as is observed in chick (Bovolenta et al., 1997). In contrast, Xenopus Otx2 does not appear to be limited to the developing RPE only during optic cup formation (Perron et al., 1998; Viczian et al., 2003). Its transcript is found throughout the developing neural and pigmented retina except the most peripheral regions, the CMZ (Viczian et al., 2003). Another difference is that while mouse Otx2 is expressed by both bipolar and photoreceptor cells (Baas et al., 2000; Nishida et al., 2003), Xenopus Otx2 is detected only in bipolar cells but not photoreceptors in the mature retina (Kablar et al., 1996; Perron et al., 1998; Viczian et al., 2003). Studies in amphibians and other species have suggested that the retinal primordia are located in the anterior neural plate and the early events of retinal field determination take place before the first morphological indication of eye formation, the bilateral protrusion of the anterior forebrain at neural tube stages (Brun, 1981; Chow and Lang, 2001; Eagleson et al., 1995; Eagleson and Harris, 1990; Martinez-Morales et al., 2004; Nieuwkoop, 1963; Zuber et al., 2003). The progressive determination of the eye/retinal primordium by complex interactions of a number of transcriptional regulators then, further establishes functionally specialized retinal compartments later: the optic stalk, the neural retina and the retinal pigmented epithelium (Chow and Lang, 2001; Zuber et al., 2003). Therefore, the 
different developmental patterns of Otx2 expression in Xenopus and mice described above imply that, depending on the species, this gene might have a different role in eye determination and/or be involved in the specification of different subdivisions of the eye primordium. Intriguingly, the expression of another member of the otd/Otx family in Xenopus, Otx 1 seems to be specifically confined to the prospective RPE field of the optic vesicle, unlike Otx2, at low levels (Kablar et al., 1996) even though there is only limited information on the expression of Xenopus Otx1. In mice, both Otx 1 and Otx2 are clearly localized to the presumptive RPE (Martinez-Morales et al., 2001). Therefore, it also can be postulated that different functional coordination of the two genes in different organisms might be responsible for controlling some aspects of eye development.

In spite of the distribution of Otx2 expression in the developing eye at critical stages, analysis of Otx2 (and also Otx1) mutant mice has provided only limited information about how the gene functions during ocular development. Complete deletion of Otx2 function in knockout mice generated by several different groups resulted in the absence of rostral head and embryonic lethality due to gastrulation defects (Acampora et al., 1995; Ang et al., 1996; Matsuo et al., 1995). In addition, microinjection of mRNA of a dominant inhibitory form of Otx2 into early Xenopus embryos also resulted in severe abnormalities in anterior neural structures, as well as the loss of eyes (Isaacs et al., 1999). Thus, analysis of tissue-autonomous effect of the mutation in $O t x 2$ on the eye tissue is greatly hindered. Interestingly, while some Otx2 heterozygous mutant mice are normal (Acampora et al., 1995; Ang et al., 1996), depending on the genetic background (Hide et al., 2002), strains of other Otx2+/- mice displayed a microphthalmia or anophthalmia 
phenotype including the absence of lens and cornea, suggesting disturbed development of the optic cup (Matsuo et al., 1995).

Drosophila otd had been reported to be required for photoreceptor R-cell development in fruit flies (Matsuo et al., 1995). However, only very recently, during the course of this study, have lines of evidence from different sources supported the idea that Otx2 gene may be directly involved in vertebrate eye formation (Martinez-Morales et al., 2001; Nishida et al., 2003; Viczian et al., 2003).

Nishida et al. (2003) ablated Otx2 in developing photoreceptors from the late optic cup stage by a Cre-lox conditional gene targeting in mice, resulting in a complete loss of retinal photoreceptors. These results suggest that Otx2 may also play a later role in the determination of photoreceptor cell fate.

Results from lipofection analysis of Xenopus retinal precursors with either wildtype Otx2 or its dominant negative form suggested that this gene product promotes bipolar cell fates (Viczian et al., 2003). Interestingly, a coinjection experiment using a closely related member of the same family, Otx $5 b$ showed that, unlike mouse Otx2, Xenopus Otx2 suppresses the enhancement of photoreceptor cell fate by Otx $5 b$. This implies that different functional interactions between Otx family genes might be involved in specifying retinal cell types in different organisms.

Since Otx1 and Otx2 genes are believed to cooperate in murine ocular development, mice carrying different numbers of functional copies of Otx1 and Otx2 have been analyzed (Martinez-Morales et al., 2001). All Otx1-/-; Otx2-/+ and 30\% of Otx1-/+; Otx2-/+ displayed consistent ocular malformations, suggesting that these genes are required in a dose-dependent manner for the normal development of the eye. While 
all the ocular tissues including lens, pigment epithelium, and neural retina were affected, the failure of RPE specification was the major phenotype in Otx1-/-; Otx2+/- mutant embryos. Partial or almost complete absence of RPE appeared to be associated with an expansion of other retinal compartments such as neural retina and optic stalk. This RPE phenotype is consistent with the observation of normal mouse Otx2 expression in the presumptive RPE territory in the optic vesicle as well as later in the differentiated RPE in mice.

Vertebrate eye formation, and particularly, the folding of the optic vesicle to form the optic cup involves complicated morphogenetic movements and cell shape changes (Hollyfield, 1973; Holt, 1980; Li et al., 2000; Matsuda and Keino, 2001; Schook, 1980a; Schook, 1980b; Schook, 1980c; Svoboda and O'Shea, 1987). In addition to the loss of the RPE tissue, the Otx1-/-; Otx2+/- mutant embryos displayed aberrant morphogenesis of the optic cup and lens (Martinez-Morales et al., 2001), raising the possibility of Otx2's involvement in controlling eye morphogenesis.

The morphogenetic abnormalities in retinal formation in these Otx mutant mice, however, could be directly due to low levels of Otx2 activity in the retina itself or indirectly due to decreased levels of OTX2 protein in the lens as an abnormal retinal phenotype has indeed been observed in a lens-specific Pax6 conditional mutant mouse (Ashery-Padan et al., 2000). Similarly, lens defects observed in Otx-deficient mice could be cell autonomous or non-cell autonomous due to the improper folding of the retina, the potential lens inducer (Furuta and Hogan, 1998). To test this hypothesis properly, the gene activity would need to be inactivated in a retina- or lens-specific manner. 
This chapter describes a study intended to clarify the direct effect of Otx2 in eye determination. I have targeted the expression of a dominant negative form of $\operatorname{Otx} 2$ to the developing eye using a retina-specific $R x$ promoter and a hormone-inducible GAL4/UAS gene regulatory system. I found that the GAL4 activator and UAS target lines I generated were able to drive a rapid induction of the dominant inhibitory Otx2-EnR effector in a temporally and spatially restricted manner. Moreover, in this experimental model, the activation of Otx2-EnR in developing retina resulted in aberrant formation of the optic cup and the lens, where Otx2 activity in the lens was not affected. The failure of proper retinal layer formation and lens differentiation resulted from the inhibition of morphogenesis of the early optic vesicle into the optic cup. Therefore, I conclude that Otx2 activity in the retina plays an important role during eye development, in particular, in morphogenesis of the optic cup and the lens. These data also demonstrate that this hormone-inducible regulatory system can be used for the study of gene function in $X$. tropicalis.

\section{Materials and Methods}

\section{DNA constructs and generation of transgenic animals}

The pCS2-Otx2 plasmid used as a template for in vitro transcription of RNA for injection (see below) was constructed by subcloning into the EcoRI-XbaI-digested pCS2 vector a fragment containing the full-length $X$. laevis Otx2 coding sequence plus a stop codon, which was PCR-amplified from pSKII/XOtx2 (a gift from K. Cho) (Blitz and Cho, 1995) by using the upstream primer 5'-CCGGAATTCAAACACTAGCATGATGTCTT 
A-3' and the downstream primer 5'-GCTCTAGATCTTCACAAAACCTGGAACTTCC$3^{\prime}$ and digested with EcoRI and $X b a \mathrm{I}$ (restriction sites are underlined in primer sequences). Amplified regions were verified by sequencing.

To make the Otx2 dominant repressor construct, pCS2-Otx2-EnR, first, Xenopus Otx2 region spanning amino acids 1-104 was PCR-amplified from pSKII/XOtx2 by using the upstream primer 5'-GCGACTAGTAACACTAGCATGATGTCTTATCTC-3' and the downstream primer 5'-GGACTAGTGCCTCCATTCTGCTGCTGC-3'. PCR products were digested with SpeI (restriction sites underlined in primer sequences) and subcloned into the SpeI-digested pCS2MT-EnR (a gift from Dr. Richard Harland) containing amino acids 1-296 of the Drosophila Engrailed protein (Conlon et al., 1996). In-frame fusion of amplified regions with the engrailed repressor domain was confirmed by sequencing. The resulting pCS2MT-Otx2-EnR was digested with ClaI and EcoRI to remove the Myc tag (MT), filled in using Klenow enzyme and re-ligated to produce pCS2-Otx2-EnR.

The pRx-Otx2-EnR construct, which was used for primary transgenic assays was generated by deleting a blunted BamHI-NotI fragment containing the EGFP coding sequences in $\mathrm{pRx}-\mathrm{GFP}$, and replacing it with a StuI-XhaI/blunt fragment containing the Otx2-EnR cassette isolated from pCS2MT-Otx2-EnR. This construct was digested with HindIII and SfiI to excise the insert from the vector (Hirsch et al., 2002) for making transgenic animals.

To construct the UAS-Otx2-EnR bicolor plasmid, a BamHI-Asp718 fragment containing the GFP cassette was deleted from GUAS-GFP3 (Bronchain et al., 1999) and replaced with a BamHI-Asp718 fragment containing the Otx2-EnR-polyA cassette from the pCS2-Otx2-EnR, creating UAS-Otx2-EnR. 
Finally, to construct the UAS-Otx2-EnR bicolor plasmid (UAS-Otx2-EnR/pCARRFP), the HindIII-AflII/blunt fragment containing the UAS-linked Otx2-EnR-polyA cassette was isolated from the UAS-Otx2-EnR plasmid and then subcloned into the pBicolor vector (see Chapter 2) by replacing a HindIII-Asp718/blunt fragment containing the ECFP cassette. This construct was then linearized with $S f i$ I for the generation of transgenic lines.

Primary transgenic embryos and transgenic lines used in this study were generated as previously described (Hirsch et al., 2002; Offield et al., 2000) with some modifications (see also Transgenesis in $X$. tropicalis at $X$. tropicalis website; http://minerva.acc.Virginia.EDU/ develbio/trop/).

\section{RNA microinjecition and RT-PCR analysis}

For RNA injection experiments, capped synthetic mRNA was transcribed in vitro from pCS2-Otx2, pCS2-Otx2-EnR, and pCS2-GFP (a gift from D. Turner) template DNA, all linearized with NotI, using SP6 polymerase according to the procedure of Green et al. (1983). Animal cap assays were carried out as described by Gammill and Sive (2001) with slight modifications. Embryos were dejellied 20-30 minutes after in vitro fertilization and placed in 4\% Ficoll (FisherBiotech) in $0.5 \times$ Modified Barth's Saline (MBS). Both blastomeres of two-cell stage wild-type Xenopus tropicalis embryos were injected with $500-600 \mathrm{pg} / 2.5 \mathrm{nl}$ of RNA(s) per injection. Injected embryos were allowed to recover for one $\mathrm{hr}$ in $4 \%$ Ficoll in $0.5 \times \mathrm{MBS}$, and cultured in $0.1 \times$ MBS until uninjected control embryos reached stage 9. Animal cap microdissections of stage 9 embryos were performed in $1.0 \times \mathrm{MBS}$ using the Gastromaster (XENOTEK 
Engineering). The explants were cultured in $0.5 \times$ MBS. Total RNA was then isolated from pools of ten stage 15 animal caps using RNeasy mini kit (Qiagen Inc.) according to the manufacturer's instructions. The protocol for RT-PCR was similar to that outlined in Gammill and Sive (2001) except that radioactive materials were not included in PCR reactions. After treatment with RNAse-free DNAse I (Roche) to remove contaminating genomic DNA, first-strand cDNA synthesis was performed by reverse transcription with random hexamers $(0.1 \mathrm{mg} / \mathrm{ml}$; Roche). Sequences for $X$. tropicalis $X A G$ - and $E F-1 \alpha$ (Krieg et al., 1989) specific primers used for PCR were obtained from the JGI Xenopus tropicalis genome project Blast site (http://genome.jgi-psf.org/cgibin/runBlast? $\mathrm{db}=\mathrm{xenopus0)}$ and the Sanger Institute Xenopus EST Blast Server site (http://www.sanger.ac.uk/cgi-bin/blast/submitblast/x_tropicalis) using the corresponding X. laevis cDNA sequences (Gammill and Sive, 2001) as bait. The oligos used for PCR were as follows: $X A G$ primers 5'-CTGACTGTCCGATCAGAC-3' and 5'-ACTGGTCA GTGCTGTGTG-3'; EF-1 $\alpha$ primers 5'-GCCACACAGCTCACATTGCTTGC-3' and 5'TCTGCCTCATGTCACGGACAGC-3'. PCR amplification of EF-1 $\alpha$ was performed to determine the relative amount of cDNA in each sample. Subsequent PCR was performed using normalized amounts of template. Serial dilutions of cDNA from explants expected to give the maximal signal were analyzed to determine the number of cycles that provides a linear range of expression for each primer pair. $X A G$ and $E F-1 \alpha$ were found to be linearly expressed with 25 cycles of amplification. Cycling conditions were: $94^{\circ} \mathrm{C}, 3$ minutes then $94^{\circ} \mathrm{C}, 30$ seconds; $55^{\circ} \mathrm{C}, 1$ minute; $72^{\circ} \mathrm{C}, 30$ seconds, for 25 cycles and ended with a single extension step of $72^{\circ} \mathrm{C}$ for 5 minutes. The RT-PCR products were resolved on a 4\% SeaPlaque GTG agarose gel (FMC BioProducts). 


\section{RU486 treatments and kinetics assays}

Experiments involving treatment with RU486 (BIOMOL Research Labs, Inc.) were carried out essentially as described in the Materials and Methods from Chapter 2. Embryos expressing activator and effector transgenes were produced by crossing a UASOtx2-EnR transgenic effector founder to a pRx-GALPR activator animal. Embryos were cultured in 0 (solvent control) or $25 \mathrm{nM}$ RU486 from st 11/12 onward and then analyzed for $O t x 2$-EnR-induced phenotypes. For kinetics assays, embryos were fixed after for 3, 6, 10,14 , and $30 \mathrm{hr}$, respectively, following administration of RU486 at st 11/12, for further in situ hybridization analysis. For testing whether DMSO can increase uptake of RU486, embryos were cultured in 1\% DMSO with 0 or $25 \mathrm{nM}$ RU486. Embryos showed phenotypes indistinguishable from those obtained by using very low DMSO concentration (insignificant concentration of DMSO; the 100\% DMSO stock initially used to dissolve RU486 was $\sim 2.3 \times 10^{6}$ times diluted in the final culture medium; see also Fig. 2-4).

\section{In situ hybridization}

Whole-mount single and double in situ hybridization was performed according to the procedure of Sive et al. (2000). Digoxigenin (Roche)-labeled and fluorescein-labeled antisense RNA probes were generated using the following cDNAs as templates, by linearization of the plasmids with the indicated restriction enzymes, and in vitro transcribed with the indicated RNA polymerase. Xrx-1 (Casarosa et al., 1997) with BamHI and T7 RNA polymerase; XOtx2 (Blitz and Cho, 1995) with EcoRI and T7 RNA polymerase; XPax6 (Hirsch and Harris, 1997) with XbaI and T7 RNA polymerase: 
pCSMT-EnR (a gift from Dr. R. Harland) (Conlon et al., 1996) with SpeI and T7 RNA polymerase. Digoxigenin-labeled RNA probes used in single in situ hybridization were revealed using BM purple (Roche) alkaline phosphatase substrate. For double in situ hybridization, after the first color reaction was done with BCIP (Roche) alone as a substrate for revealing fluorescein-labeled RNA probes, the remaining alkaline phosphatase was inactivated by incubating embryos for 30 minutes at $65^{\circ} \mathrm{C}$ in $10 \mathrm{mM}$ EDTA in PBS, and followed by the second color reaction with NBT/BCIP substrate to reveal digoxigenin-labeled RNA probes.

\section{Fluorescent immunohistochemistry}

For fluorescent double immunostaining, embryos were fixed in MEMFA (Harland, 1991) for 1-1.5 $\mathrm{hr}$ at room temperature or overnight at $4{ }^{\circ} \mathrm{C}$ and stored in ethanol at $-20^{\circ} \mathrm{C}$ until needed, then were rehydrated in graded ethanols and washed in PBS. Rehydrated embryos were infiltrated in $30 \%$ sucrose in PBS for $1 \mathrm{hr}$ at room temperature, embedded in OCT (Tissue-Tek, Sakura Finetechnical Co., Ltd.), frozen and sectioned on a Leica CM3050 cryostat at 10-15 $\mu \mathrm{m}$. Cryosections were washed twice for 10 minutes in $1 \times$ PBS $+0.1 \%$ Triton X-100 (PBT), blocked 1 hr with PBT $+10 \%$ heatinactivated goat serum then incubated overnight at $4^{\circ} \mathrm{C}$ with a mixture of primary antibodies: a monoclonal anti-rhodopsin antibody $(\mathrm{B} 630 \mathrm{~N})$ specific for principal rod opsin (a gift from Dr. C. Green) (Adamus et al., 1991) at a 1:4 dilution and a rabbit polyclonal anti-Pax6 antibody $(\mathrm{BAbCO})$ at a 1:30 dilution. Sections were washed in PBT four times for 5 minutes. Antibody binding was visualized by incubating the sections for 1-2 $\mathrm{hr}$ at room temperature with the mixture of the appropriate secondary antibodies: a 
goat anti-mouse IgG tetramethylrhodamine (TRITC)-conjugated antibody (Sigma) at a 1:25 dilution and a goat anti-rabbit IgG Fluorescein isothiocyanate (FITC)-conjugated antibody (Sigma) at a 1:80 dilution. The sections were washed again in PBT three times for 5 minutes then in PBS once for 20 minutes, and mounted with $10 \%$ glycerol in PBS

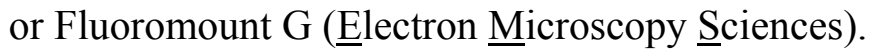

\section{Histology}

Following whole mount in situ hybridization, embryos were postfixed overnight at room temperature in MEMFA (0.1M MOPS pH 7.4, $2 \mathrm{mM}$ EDTA, $1 \mathrm{mM} \mathrm{MgSO}_{4}$, $3.7 \%$ formaldehyde) or Bouin's fixative (1\% picric acid, $9.25 \%$ formaldehyde, $0.05 \%$ glacial acetic acid). After the postfixation, some embryos were bleached in $1 \% \mathrm{H}_{2} \mathrm{O}_{2}, 5 \%$ formamide, 0.5 $\times$ SSC as described by Mayor et al. (Mayor et al., 1995) and examined as whole mounts in PBS. Some were embedded in Paraplast and sectioned at 10-15 $\mu \mathrm{m}$. Sections were mounted in Permount (Fisher) before photomicrography. For Hematoxylin staining, frozen sections were made as described above and stained by immersion in Ehrlich's hematoxylin.

\section{Photomicroscopy}

Images of whole-mount embryos were obtained using a Zeiss Stemi SV11

dissecting microscope. Sectioned samples were photographed on a Zeiss Axioskop microscope. Images were captured using a Dage MTI 330 cooled CCD camera or a Carl Zeiss AxioCam MRc 5 camera. 


\section{Results}

\section{A dominant negative form of Otx2, Otx2-EnR, inhibits Otx2 activity}

To study the role of Otx2 during eye determination, independent of its role in overall head development, I first used a transgenic strategy whereby the transcriptional activity of OTX2 was specifically blocked in the developing retina by a construct engineered to have a dominant inhibitory effect on Otx2 targets. As Otx2 may function as either a transcriptional activator or a repressor depending developmental context (Gammill and Sive, 2001; Isaacs et al., 1999; Morgan et al., 1999a; Nakano et al., 2000; also see Discussion), a dominant negative variant of $O t x 2$, Otx2-EnR that acts exclusively as a repressor was created by fusing the Otx 2 homeodomain to the Drosophila engrailed repressor domain (Conlon et al., 1996; Gammill and Sive, 2001; Isaacs et al., 1999; Viczian et al., 2003). The specificity of this dominant negative construct in Xenopus has been assessed by several different methods (Gammill and Sive, 2001; Isaacs et al., 1999; Viczian et al., 2003; reviewed in more detail in Discussion). In order to evaluate the effectiveness of the Otx2-EnR construct, I took advantage of the observation previously made by Gammill and Sive (2001). Microinjection of Otx2 mRNA has been shown to activate the ectopic expression of its early target genes, cement gland-markers $X C G$ and $X A G$ (Gammill and Sive, 1997; Gammill and Sive, 2001) in whole embryos or animal cap explants. Therefore, I first examined the ability of the Otx2-EnR construct to interfere with endogenous Otx2 activity in an animal cap assay (Fig. 3-1) before I tested this construct in transgenic frogs. In vitro transcribed mRNAs were microinjected into embryos at the two-cell stage, and animal caps were dissected at late blastula stage (stage 
9) and cultured until mid-neurula (stage 15). The expression of a cement gland marker $X A G-1$ in the animal caps was then assayed by RT-PCR (Fig. 3-1B). Full-length wildtype Otx2 induced the activation of $X A G-1$ expression (compare lane 1 and 2 in Fig. 31C). This activation was almost completely suppressed when Otx2 was coinjected with Otx2-EnR (compare lane 2 and 4) although effective suppression seemed to required higher amount of Otx2-EnR than Otx2 (at a molar ratio of 7.5:1; see Discussion). These results showed that Otx2-EnR effectively inhibited the function of Otx2 gene product.

\section{Targeted inactivation of Otx2 and localized overexpression of Pax6 in primary transgenic animals}

To study the in vivo effects of targeted Otx2 inactivation, Otx2-EnR was cloned downstream of the retina-specific Xenopus tropicalis $R x$ promoter (see Fig. 2-2 in Chapter 2). The resulting chimeric construct, pRx-Otx2-EnR (Fig. 3-2A) was linearized and utilized to generate transgenic Xenopus embryos. As a control, transgenic embryos were also generated with the $R x$ promoter driving GFP (pRx-GFP). The embryos generated with pRx-Otx2-EnR showed specific defects in developing eyes whereas a higher percentage of embryos bearing pRx-GFP is apparently normal (Fig. 3-2). The abnormal eye phenotype of these embryos is discerned at stage $40 / 41$ by the abnormal RPE tissue between the eyes and the neural tube (arrows in Fig. 3-2B). The mild phenotypes consist of very thin short RPE extended from eye that points toward the brain (Fig. 3-2B-b, c). In more severely affected embryos entire eye structure including RPE remains abnormally continuous with the forebrain (Fig. 3-2B-d). 
When generating transgenic frog embryos using our standard transgenic procedure, not all resulting primary embryos express the transgene. Given that a range of 30 to $60 \%$ of embryos, on average, is transgenic based on the experiments using GFP reporter constructs, the fraction of embryos expected to carry the transgene and thus display the abnormal phenotype is also 30 to $60 \%$. When scored at stage $41,26.8 \%$ of embryos generated with pRx-Otx2-EnR displayed the eye defects (Fig. 3-2C). However, the eye phenotype was also detected in $13.2 \%$ of control embryos bearing the EGFP reporter construct. This difference in producing eye phenotype is not statistically significant between the two constructs as determined by a $\chi^{2}$ test $(p>0.05)$. In addition, these numbers were obtained by combining data from several different experiments and the percentage of embryos showing the abnormal eye phenotype varied from experiment to experiment. The high "background" eye abnormalities in control embryos $(13.2 \%$ compared to $26.8 \%$ with Otx2-EnR-generated embryos) indicate that non-specific developmental abnormalities associated with our nuclear-transfer-mediated transgenic procedure might obscure the phenotypic analysis in primary transgenic embryos. The embryos with very severe gastrulation defects including partial or complete loss of the anterior structures (data not shown) were not scored as eye defects (e in Fig. 3-2C; $n=14$ (13.0\%) with $\mathrm{pRx}-$ Otx2-EnR and $\mathrm{n}=3$ (5.7\%), with $\mathrm{pRx}-\mathrm{GFP}$, respectively). These anterior phenotypes obscuring the eye phenotype added another complication to the interpretation of the phenotypic effects of the transgene.

To test whether the embryos displaying the eye defects are transgenic, genomic PCR analysis was performed using genomic DNA isolated from the embryos generated with pRx-Otx2-EnR. The primers utilized were designed to detect only the pRx-Otx2- 
EnR transgene, not the endogenous $R x$ or Otx2 locus (Fig. 3-2A). All embryos displaying the eye phenotype were found to be pRx-Otx2-EnR-positive. However, the PCR product was also amplified from approximately a half of the normal embryos. This indicates that the transgene might exist as a plasmid without being incorporated into the genome and/or there are variations in the level of transgene expression in each embryo representing a unique insertional event. This further complicates scoring the phenotype obtained from primary transgenic method.

These data indicate that the functional inactivation of Otx2 transcriptional activity affects the eye development. However, background defects resulting from transgenic technique itself might hamper the analysis of the phenotype. To further assess the usefulness of primary transgenesis for this kind of experiment, I tested the utility of the primary transgenic approach for the phenotypic analysis by using a gene, $\operatorname{Pax} 6$, which has predictable expected effects on eye development when overexpressed. Transgenic mice carrying multiple copies of Pax6 displayed a unique ocular defects including microphthalmia (Schedl et al., 1996). To test the proposal that changes in Pax6 levels may cause developmental abnormalities of the eye in the frog, a similar transgenic approach was employed to introduce additional copies of the Pax6 gene into Xenopus genome. In order to target Pax6 expression specifically to developing retina, the $R x$ promoter was used. A construct expressing Pax 6 under the control of the $R x$ promoter, pRx-Pax6 was utilized to generate transgenic embryos. pRx-GFP was also introduced into embryos as controls. However, I was unable to confirm eye phenotype in the resulting embryos (data not shown) as variability of the phenotype and transgenic 
procedure-associated background abnormalities including defects in anterior development hindered a statistical analysis of the phenotype.

\section{Functional inactivation of Otx2 in the retina by a hormone-inducible GAL4/UAS system causes an ocular phenotype}

To overcome the difficulties of the primary transgenic strategy described above, the ligand-inducible GAL4/UAS system adapted for $X$. tropicalis (see Chapter 2) was utilized. Two separate transgenic lines were generated to determine Otx2 function during eye development by repressing Otx2 activity in the retina through the induced expression of the dominant inhibitory form of Otx2. UAS-Otx2-EnR effector lines carry a UASdriven chimeric construct, Otx2-EnR, linked to a second reporter cassette, pCAR-RFP to mark transgenic embryos (see Chapter 2). The pRx-GALPR lines drive the expression of the GALPR itself and the UAS-ECFP reporter in the developing retina (Chapter 2). I used these GALPR transactivator lines to express the Otx2-EnR from the UAS-Otx2-EnR lines.

Embryos from this cross between a pRx-GALPR line and a UAS-Otx2-EnR line were raised in the presence of 0 or $25 \mathrm{nM}$ RU486 to activate Otx2-EnR expression. Expression of the Otx2 gene in the presumptive retinal region of the early neurula implies that its activity might be important for retinal development from early neural plate stages. Therefore, RU486 was administered from stage 11/12 onward to ensure that early Otx2 activity for retinal formation is repressed in this assay. Since I showed earlier that the GALPR activator from the pRx-GALPR line is expressed as early as st 12 (see Fig. 2-6), it should be activated by RU486 administered from this stage onward. As described for 
the cross of pRx-GALPR to UAS-ECFP lines in Chapter 2, each of the activator and effector lines in this cross is hemizygous for a transgenic locus. The cross produces four different genotypes: non-transgenic embryos, embryos bearing one transgene (either the UAS-Otx2-EnR effector or GALPR transactivator gene), and embryos bearing both effector and transactivator transgenes. Only double transgenic embryos treated with RU486 showed specific eye defects (Table 3-1; Fig. 3-3B, C) while their axial body structures including the posterior neural tube appeared to be normal. Embryos showed no abnormal phenotype in the absence of RU486 (Table 3-1; Fig. 3-3B, C). As shown in Table 3-1, $40 \%$ of the RFP-positive (RFP+) embryos displayed an eye phenotype in the presence of $25 \mathrm{nM}$ RU486. RFP-negative (i.e. UAS effector-negative) embryos displayed apparently normal eyes. It should be noted that at most $50 \%$ of embryos carrying the UAS-Otx2-EnR target gene (i.e. RFP+) are expected to carry the GALPR transactivator gene as well, thus developing the phenotype. PCR genotyping of individual embryos was carried out using GALPR-specific primers and all 11 of the tested embryos displaying the abnormal eye phenotype were found to carry the GALPR transgene in addition to UAStarget gene (data not shown).

The morphological consequence of Otx2-EnR expression in the retina ranges from incomplete optic fissure closure to greatly reduced eye structures. Representative embryos from this assay are shown in Fig. 3-3. The abnormal eye phenotype of RU486treated embryos was noticeable superficially as early as swimming tadpole stages (st 37/38), by a failure to close the optic fissure (arrows in Fig. 3-3E). The eye defect becomes more obvious at stage 40/41. Embryos showing abnormalities in the eye at this stage can be divided into two groups although the severity of the phenotype varies within 
each group. One group of embryos (* in Fig. 3-3D) displayed a mild eye phenotype typified by a typical wide-open optic fissure whose borders do not contact each other (arrows in Fig. 3-3D). The eye defects in the other group (** in Fig. 3-3D) were more severe. From the lateral view, a small patch of pigmented tissue constituted the only remnant of external eye structures in some cases (arrowheads in Fig. 3-3D).

Dorsal views of the embryos with the mild phenotype revealed that RPE tissue is frequently present in the form of cells extending from the eye towards the brain (arrow in Fig. 3-3H). In severely affected embryos, I observed a morphologically abnormal eye cup (arrow in Fig. 3-3G) which was broadly attached to the anterior neural tube. In most cases, the abnormal optic cup was greatly reduced in size compared to untreated embryos. In wild-type embryos, the optic cup and the forebrain are connected only by the very thin inconspicuous optic nerve (data not shown; arrows in Fig. 3-3F and I). These results are consistent with the phenotypes observed in the relatively severely affected primary transgenic embryos generated with the pRx-Otx2-EnR construct described above.

In addition, the distance between the neural tube and the distal end of optic cup, and thereby the width of the head region encompassing the eye, was significantly reduced in embryos displaying the more severe phenotype when compared with that of control embryos (Fig 3-3I). This implies that the pigmented tissue present between the eye and the brain is not simply a misplaced ectopic RPE. Instead, early morphogenesis responsible for the 'out-pocketing' of the optic vesicle from the anterior neural tube and/or subsequent retinal cell movement might be disrupted. Despite abnormalities in the eye, most of these embryos showed relatively normal brain morphology although marker 
genes for different brain regions need to be examined to assess subtle changes in their brain structures.

These data indicate that our activator and UAS-Otx2-EnR target lines used can drive RU486-induced expression of Otx2-EnR to levels capable of efficiently repressing the endogenous Otx2 activity retina-specifically and producing the eye phenotype.

\section{Otx2 activity is required for eye morphogenesis}

Although the eye phenotypes resulting from the induced, retina-specific repression of Otx2 suggest a role for Otx2 in eye morphogenesis, questions remain about the specific nature of Otx2 function and the morphogenetic process affected. One question is whether the ectopic eye tissue is comprised only of pigmented tissue connecting the eye and the brain includes only the RPE or other retinal tissues such as neural retina. A second question is whether this pigmented tissue results from ectopic retinal tissue formation at the expense of the region lying between the neural tube and the eye, or from abnormal cell morphogenesis of the primary optic vesicle and/or optic cup.

To address these questions and to understand morphogenetic mechanism responsible for the eye phenotype described above, histological analysis of these embryos was performed. Cross sections of the RU486-treated embryos at several different developmental stages (Fig. 3-4A-E and G-I) show that morphogenesis of optic vesicle and optic cup is strongly affected in embryos expressing Otx2-EnR. During normal development in Xenopus, the eye primordium arises as a lateral outpocketing of the anterior neural tube and becomes optic vesicle at stage 22 (Holt, 1980; Nieuwkoop and Faber, 1967). The epithelium of the vesicle remains connected with the neural tube 
through a continuous lumen until the optic cup stage (see Fig. 4-1). Later, the vesicle elongates dorsally (Fig. 4-1A and B) during the optic vesicle to optic cup transition. Cross sections of the st 22 optic vesicle display a typical wing-shaped epithelium connected with the forebrain (data not shown; right half in Fig. 3-4A). The formation of optic vesicle by bilateral protrusion appeared to be initiated in the Otx2-EnR-expressing embryos, although it displays unique morphological abnormalities. The left and right eye from a single embryo sometimes exhibited different levels of the severity of the phenotype. In some cases, one eye appeared almost normal (red open arrow in Fig. 3-4A) whereas the dorsal protrusion and elongation of the eye vesicle on the other side were strongly disrupted (black open arrow in Fig. 3-4A).

In wild-type embryos, as the eye vesicle elongates dorsally, the vesicle remains connected with the brain by the very narrow optic stalk (arrowheads in Fig. 3-4B; also see Fig. 4-1B) only at its ventral pole. The invagination of the distal region of the vesicle, the presumptive neural retina, then begins dorsally at st 27 (open arrow Fig. 4-1B) and brings itself adjacent to the proximal wall of the vesicle, the future RPE, which has become an elongated monolayer of flattened cells (arrow in Fig. 3-4B; Hollyfield, 1973; Holt, 1980). These morphogenetic movements and consequently, the folding of the optic vesicle into the optic cup are severely affected in Otx2-EnR-expressing embryos. Figure $3-4 \mathrm{C}$ illustrates the typical ocular phenotype of embryos expressing Otx2-EnR during early optic cup formation. The thick epithelium of the abnormal eye vesicle (black arrow in Fig. 3-4C) has not elongated, probably a result of the disruption of normal movement of the vesicle, which remained connected with the neural tube as in the younger stage optic vesicle shown in A. The cavity of the eye vesicle (open arrow in Fig. 3-4C) was 
abnormal, retaining its continuity with the wide lumen of the neural tube $(*)$. In addition, lens placode formation appeared to be delayed in these embryos as the thickening of the overlying surface ectoderm (open arrowheads in Fig. 3-4B and C) was not observed at this stage in Otx2-EnR-expressing embryos.

The optic fissure develops as a vertical cleft on the ventral side of the invaginating optic vesicle. Its formation begins in the distal margin (arrowhead in Fig. 4-1B) of the vesicle at st 29 (Arey, 1954; Chow and Lang, 2001; Feduccia and McCrady, 1991; Grant and Rubin, 1980; Holt, 1980; Lopashov and Stroeva, 1964). As the retinal invagination deepens proximally (open arrow in Fig. 4-1B) and ventrally, the fissure also extends proximo-ventrally as a groove on the underside of the optic stalk. For a time, the choroid fissure remains open. The optic fissure eventually closes after st 36 so that the inner (proximal) sensory layer of the optic cup is continued directly within the optic stalk. This continuity provides a tunnel through which the optic nerve fibers growing out of ganglion cells in the retina pass to the brain. The choroid fissure is nearly closed (Nieuwkoop and Faber, 1967) at st 35/36.

It has been argued that the lower (ventral) half of the eye cup begins to develop by the downward movement (arrows in Fig. 4-1C) of retinal cells and gradually grows to the size of the dorsal half (Lopashov and Stroeva, 1964), as the fissure is closed. Note that the ventral margin of the eye before this movement is at the same level as that of the neural tube as shown in Fig. 3-4 B, but later the ventral part of the retina is located further below the ventral pole of the neural tube as shown in Fig. 3-4D.

The ventral RPE of the st 37/38 Otx2-EnR-negative control embryo was formed (arrowhead in Fig. 3-4D), presumably as a result of the ventral movement described 
above. The darkly pigmented RPE, the outer wall of the optic cup, encircled the entire neural retina, except the opening of the cup to the exterior through the lens. The cavity of the optic vesicle has completely disappeared as the inner and outer layers of the cup become apposed. The double-layered optic cup has clearly separated from the neural tube even in the anterior sections of control embryos. In contrast, at a similar plane of sectioning, Otx2-EnR-expressing embryos still showed the presence of a wide central lumen (open arrows in Fig. 3-4E) connecting the forebrain ventricle and the optic cup. The RPE was often found dorsally only (arrow in Fig. 3-4E), presumably resulting from the inhibition of the early morphogenetic elongation and the later ventral movement of the optic vesicle. This result is consistent with the fate map of the dorsal region of the early optic vesicle, which is fated to become the pigmented epithelium. The ventral RPE did not form (* in Fig. 3-4E). The optic fissure was widely open in these embryos (see Fig. 3-3). These results indicate that the retinal cell movements responsible for the optic fissure closure and the completion of the ventral retina formation are also impaired by the induced expression of Otx2-EnR in the retina of these embryos.

At st 41, the abnormal optic cup continued to fail to separate from the neural tube of GALPR+; UAS+ embryos treated with RU486 (Fig. 3-4H and I). For genotyping, PCR analysis was performed with GALPR-specific primers (data not shown). Epithelium of the optic cup of these double transgenic embryos is abnormally attached to the brain through a wide RPE-free region (open arrows in Fig. 3-4H and I). Ventral RPE was found in most of the mildly affected embryos (arrowhead in Fig. 3-4H) but was completely absent in some of the more severely affected embryos. In some cases, only a small patch of pigmented tissue delineated morphologically recognizable ventral RPE 
(arrowhead in Fig. 3-4I). This partial or complete loss of the ventral RPE indicates that the ventral movement of retinal cells and/or the further growth of the ventral retina during the final phase of the eye cup formation were severely impaired. In addition, the RPE surrounding the dorsal neural retina was found often to be considerably thicker (arrows in Fig. 3-4H and I) than control RPE, which consists of a thin layer of cells (arrow in Fig. 34G). This suggests that morphogenesis of optic vesicle responsible for the formation of a monolayer of pigmented epithelium is defective in these embryos. Several cell biological mechanisms, including the cell elongation and cell intercalation might underlie morphogenesis of a thin single layer of the RPE (see Discussion).

The embryos displaying the abnormal eye phenotype were then raised in a normal culture medium without RU486 from st 41 onward, therefore any later Otx2 function in the eye should be retained. Fig. 3-4F shows a representative three-week-old tadpole; the dorsal part of the eye is covered with a pigmented epithelium, and is directly connected with the brain (arrow in Fig. 3-4F). The part of the forebrain contacting the eye was pigmented (arrowhead in Fig. 3-4F), presumably either by the invasion of the pigmented retinal tissue onto the surface of the brain, or by the conversion of that part of the brain into pigmented epithelium. The distal tip of the eye did not contact the surface ectoderm, in contrast to the wild-type tadpole at this stage (data not shown). The length of the eye structure extended from the brain varied from embryo to embryo (data not shown) and sometimes even between left and right sides of a single embryo. The degree of separation of the optic cup from the brain was also variable, as shown in Fig. 3-4F (arrowhead vs. open arrow). Other anterior structures such as nose (N in Fig. 3-4F) and ear (E in Fig. 34F) appeared normal. 
The eye phenotype of metamorphosed froglets ranges from a protrusion of the pigmented tissue (arrow in Fig. 3-4K) to no visible eye structures except a small indentation (arrow in Fig. 3-4L) in the region where the eyes normally are positioned (Fig. 3-4J). Superficial eye structures such as lens, iris and cornea were not observed in these animals. Moreover, when these froglets were raised to sexual maturity and outcrossed to the wild-type animals, resulting embryos (i.e. $F_{2}$ embryos of pRx-GALPR and UAS-Otx2-EnR founder animals) reproduced the same ocular phenotype, only in the presence of RU486 (data not shown). As each of the adult animals carries both the GALPR transactivator and UAS effector constructs and thus $25 \%$ of progeny of each animal are expected to also carry both transgenes, only the expected fraction of embryos from this outcross showed the phenotype. Untreated embryos were indistinguishable from wild-type control embryos, demonstrating that the induction of the phenotype is RU486-dependent and not from insertional damage of the genome by the transgenes. This also suggests that this inducible gene regulatory system is suitable for multigeneration experiments.

These phenotypes indicate that functional repression of the transcriptional activity of Otx2 gene product within the presumptive retina results in disruption of morphogenesis required for early optic vesicle and optic cup formation and thus Otx2 plays an important role in these developmental processes.

\section{Kinetics of induction of Otx2-EnR transgene expression}

The ocular phenotype obtained by the induced expression of the Otx2-EnR transgene indicates that the activity of the Otx2 gene product during early ocular 
development is important for morphogenesis of the optic cup. In order to gauge more precisely when the dominant negative gene product is active, I next asked how early the transgene is activated upon the administration of RU486 and thus could start functionally inhibiting the transcriptional activity of OTX2 in the eye. In order to address the retina specificity and the kinetics of Otx2-EnR transgene expression in response to RU486, in situ hybridization analysis was performed using embryos from the cross of pRx-GALPR and UAS-Otx2-EnR animals. After 3, 6, 10, 14, or $30 \mathrm{hr}$ of incubation in $25 \mathrm{nM}$ RU486 from st $11 / 12$, the embryos were analyzed for the Otx2-EnR transgene expression by in situ hybridization using a probe specific for the EnR domain.

The Otx2-EnR transgene expression was mainly localized in the developing retina of RU486-treated embryos (Fig. 3-5) and indistinguishable from the reporter expression mediated by pRx-GFP under the same promoter (see Fig. 2-2). Solvent-treated control embryos showed very low Otx2-EnR transcriptional activity (data not shown). In addition, the RU486-mediated transgene induction was rapid. The Otx2-EnR transcript was detectable within $3 \mathrm{hr}$ after application of RU486 (Fig. 3-5A). However, when embryos reached st 28-30 after $30 \mathrm{hr}$, the Otx2-EnR expression became localized as punctate clusters dispersed throughout the retina (Fig. 3-5E, F). Later in development, the expression was detected as smaller patches of staining within the retina (data not shown; see Fig. 3-4E) as with the ECFP expression from UAS-ECFP driven by the same activator (see Fig. 2-5). The GALPR transcript driven by the $R x$ promoter in $\mathrm{pRx}-$ GALPR embryos was uniformly present in the neuroretina at stages $28-30$ or in the retinal ciliary margin at later stages as detected by in situ hybridization analysis (Fig. 26). Therefore, the GALPR transgene does not seem to contribute to the observed patchy 
expression of the effector. As discussed in detail in the Discussion in Chapter 2, this could be due to the cell-to-cell variability in response to the GAL4 activator as has been seen in Drosophila or due to a positional effect of the effector transgene integration.

These data indicate that a simple application of RU486 by culturing embryos in a medium containing the ligand rapidly activates $O t \times 2$-EnR expression, thereby potentially repressing the OTX2 activity from early neurula stage. The expression pattern of Otx2 suggests that its activity might be important for eye development from early neural plate stages (Zuber et al., 2003).

\section{RU486-mediated Otx2-EnR expression overlaps with expression of its target, Otx2, in developing retina}

For Otx2-EnR to be able to dominantly inhibit OTX2 activity, it must be present where its target, Otx2 is expressed. Therefore, I next asked if expression of Otx2, specially in the early eye territories is overlapping with that of its dominant inhibitory Otx2-EnR which is driven by the $R x$ promoter.

Expression domains of Xenopus Otx2 include presumptive fore-/mid-brain and eye field in the anterior neural plate (Andreazzoli et al., 1999; Blitz and Cho, 1995; Kablar et al., 1996; Pannese et al., 1995). This expression pattern dynamically changes during neurulation along with the morphogenesis of the neural plate into the neural tube. $R x$ expression is mainly confined to the prospective eye field in the most anterior part of the neural plate and subsequently in the neural structures of the eye (Andreazzoli et al., 1999; Casarosa et al., 1997). 
First, to determine the relative expression patterns of $O t x 2$ and $R x$ (i.e. Otx2-EnR) at neural tube stage when Otx2 is clearly expressed in the presumptive eye, double whole-mount in situ hybridization was performed. I first compared the expression domain of Otx2-EnR with $R x$. The Otx2-EnR expression pattern was identical to that of $R x$ in a st 19 single embryo hybridized with probes for $R x$ and the EnR repressor domain (compare A and $\mathrm{A}^{\prime}$ in Fig. 3-6), suggesting that the $R x$ promoter in the pRx-GALPR line indeed recapitulates the endogenous $R x$ patterning. There appeared to be no obvious alteration in $R x$ expression in the Otx2-EnR expressing embryos when compared with that of Otx2EnR-negative embryos (compare A' and B'). Double in situ hybridization of a st 22/23 embryo with Otx2 and EnR probes revealed that Otx2-EnR expression (arrow in Fig. 36C) was largely overlapping with Otx2 within the presumptive retinal field (black arrows in Fig. 3-6C'). Otx2 expression did not appear to be affected in Otx2-EnR-expressing embryos (compare $\mathrm{C}^{\prime}$ and $\mathrm{D}^{\prime}$ in Fig. 3-6). These results showed that Otx2 expression in the evaginating eye vesicle during the neural tube and early tailbud stages is largely overlapping with Otx2-EnR transgene expression, presumably leading to the suppression of the OTX2 activity.

Although I confirmed that Otx2 expression is overlapping with the induced Otx2EnR expression in the developing retina during early optic vesicle stages, it was not clear whether this overlapping starts in the presumptive retinal field at earlier stages, as Xenopus Otx2 displays a highly dynamic pattern of expression during early- and midneurula stages. Thus, it was necessary to define, in more detail, the spatial relationship between expression domains of Otx2 and Otx2-EnR (and $R x$ ) genes from early neurula stage to interpret phenotypic consequences observed later. Therefore, the expression 
domains of these genes were determined by double in situ hybridization. As previously reported in X. laevis (Andreazzoli et al., 1999; Kablar et al., 1996; Pannese et al., 1995; Zuber et al., 2003), X. tropicalis Otx2 was expressed in the entire presumptive anterior neuroectoderm at the end of gastrulation (stage 12; Fig. 3-7A). At this stage, Rx (Fig. 37E) transcripts were first detected within the Otx2 expression domain. Induction of Otx2EnR (Fig. 3-7B) expression was detected in a very small portion of RU486-treated embryos tested at this stage (st 12) as most of embryos might not be exposed long enough to activate Otx2-EnR expression by RU486 administered at st $11 / 12$ and there is a potential time delay between the administration and the transactivation of an effector. Subsequently at stage $12.5-13$, there was a rapid reduction in Otx2 expression in the medial region of its expression domain (arrow in Fig. 3-7C). As seen in wholemount, Otx2-EnR (Fig. 3-7D) and $R x(\mathrm{~F})$ expression sharply delineated the inner limits of this 'hole' in the Otx2 expression domain, which represents the presumptive retinal field (Eagleson et al., 1995; Zuber et al., 2003). Otx2 and Rx (and Otx2-EnR) expression domains appeared to remain mutually exclusive by midneurula stages (stage 14/15). Subsequently at st $15 / 16$, these domains were overlapping in part within the region fated to give rise to the retina (arrowheads in Fig. 3-7I and M; Eagleson et al., 1995; personal communication with Eagleson, J). During subsequent development of the neural tube, as Otx2 was detected in the anterior brain and the developing eye anlage, $R x$ and Otx2 domains appeared to be largely overlapped in the presumptive retina (Fig. 3-7K and M). In summary, Otx2 and $R x$ promoter-driven Otx2-EnR expression domains do not appear overlapped at early neurula stage (st 12) as Otx2-EnR expression by RU486 administered at st 11/12 has not been induced in most of embryos at this stage. The two expression 
domains remain almost mutually exclusive at neural plate stages (st 12.5-14) but they start partially overlapping in the presumptive retinal field at subsequent stages (st 15/16). Finally, the domains largely overlap in the presumptive retina later at neural tube/tail bud stages. These results indicate that relative expression domains of $O t x 2$ and $R x$ promoterdriven Otx2-EnR started overlapping in the future retina after midneurula stages, thus enabling repression of the OTX2 activity before the formation of the optic vesicle.

\section{Altered gene expression and retinal cell differentiation in the optic vesicle and optic cup of embryos expressing Otx2-EnR}

To test whether the morphological abnormalities observed in Otx2-EnRexpressing eye early in development affects the retinal cell fate decision and differentiation as well as corresponding patterning later, I have analyzed the expression of genes important for general eye development and specific retinal cell differentiation, such as Pax6 and opsin.

First, I examined whether there might be an alteration in the expression of Pax 6 at critical stages during the folding of the optic vesicle into optic cup. Pax6 was chosen because it is expressed throughout the developing retina at early stages and later in specific retinal layers. Thus it serves as a good marker for cells fated to form the eye and for certain differentiated retinal cells in late optic cup stages. The distribution of Pax6 was analyzed together with that of Otx2-EnR by double in situ hybridization using Pax6 and EnR probes. In this way, I was able to 1) identify the embryos expressing Otx2-EnR and 2) compare Pax6 expression in these Otx2-EnR-positive and control sibling embryos simultaneously. In Otx2-EnR-negative control embryos at st 31/32 (Fig. 3-8D), Pax6 
mRNA was found in parts of forebrain, hindbrain, and the spinal cord with a gap in the mesencephalon (the region between two arrowheads in Fig. 3-8D'), as well as in the developing eye (arrow in Fig. 3-8D'). In embryos expressing Otx2-EnR, the expression of Pax6 transcript in the eye seemed to be largely overlapping with that of the Otx2-EnR transgene driven by the retina-specific pRx-GALPR driver (white arrows in Fig. 3-8A and $\mathrm{A}^{\prime}$ ). From the whole mount view, the Pax6 expression pattern appeared unchanged (compare $\mathrm{A}^{\prime}$ and $\mathrm{D}^{\prime}$ in Fig. 3-8) and the eye did not seem to be severely disrupted morphologically. However, transverse sections of the same embryos revealed changes in Pax6 expression in addition to morphological defects in the eye. In control embryos, Pax6 transcript became localized to the presumptive neural retina. Moreover, the intensity of Pax 6 expression was decreased in a specific layer, the photoreceptor or outer nuclear cell layer (o in Fig. 3-8E and F), as previously reported in Xenopus laevis (Hirsch and Harris, 1997). In contrast, Pax6 transcripts were more uniformly distributed at slightly lower level throughout the retina of Otx2-EnR-expressing embryos (Fig. 3-8B and C). Interestingly, the dorsal part of the abnormal retina, which would have become the RPE otherwise, showed relatively weaker expression of Pax6 (arrowheads in Fig. 38B and $\mathrm{C}$ ) than more ventral region. The RPE cells of the control embryos have begun to make pigment and no longer express Pax6 mRNA at this stage.

Morphologically, the presumptive RPE has already become a thin layer of cells (black arrowheads in Fig. 3-8E and F) in control embryos at this stage. This future RPE is in contact with the neural retina moving inward from the dorsal part (red arrowhead in Fig. 3-8F; also see open arrow in Fig. 4-1B) in more posterior sections at the level of the lens. However, the dorsal part of the eye vesicle still remains as a thick epithelium 
(arrowheads in Fig. 3-8B and C) and the entire vesicle is connected with the neural tube through the continuous central lumen (open arrow in Fig. 3-8B) in anterior sections of the Otx2-EnR-expressing eye. In more posterior sections, the vesicle is still continuous with the brain epithelium although the vesicle lumen sometimes appears separated from the brain ventricle (open arrow in Fig. 3-8C). However, because of the curvature of growing optic vesicle (and the vesicle lumen in it) in a posterior direction (Arey, 1954; Feduccia and McCrady, 1991), sections cut at a more posterior level could make the lumen appear separated from the brain even though the vesicle cavity is still continuous with the brain vesicle. Horizontal sectioning would reveal the precise morphological features of these mutant eyes. The lens is either absent or often reduced in size as shown in Fig. 3-8C (open arrowhead) when compared to that of control embryos (open arrowhead in Fig. 3$8 \mathrm{~F})$.

Next, I examined the expression of Pax6 in Otx2-EnR-positive embryos at st $37 / 38$ when retinal cell nuclei are arranged in three layers in the neural retina. At this stage, the Pax6 transcript is normally limited to the ganglion cell layer and the inner nuclear layer (Fig. 3-9B). Cells in the outer nuclear layer no longer express Pax6. Pax6 expression in the lens has also changed at this stage (Hirsch and Harris, 1997); the expression level in the lens ectoderm increases initially during the formation of optic cup (also see Fig. 3-8) as the lens ectoderm thickens. From stage 33/34, Pax6 staining in the lens decreases gradually as the lens cells becomes fully differentiated. However, cells in the most distal part of the lens, the mitotic lens epithelium, continue to express Pax6 (arrowheads in Fig. 3-9B) until the completion of optic cup formation. The double in situ hybridization analysis using the probes for EnR and Pax6 revealed that the restricted 
expression of Pax6 described above is disrupted by RU486-induced expression of Otx2EnR in the retina (Fig. 3-9). Note that EnR expression in the eye becomes patchy (black arrow in Fig. 3-9I; see Discussion) at this stage and dark overlapping Pax6 expression also obscures the detection of EnR staining. For genotyping, PCR analysis of individual embryos was performed with primers specific for the coding sequence of RFP or GALPR, and only double transgenic UAS+ (RFP+); GALPR+ embryos showed Otx2EnR expression. In severely affected embryos, the Pax6 transcript was more evenly distributed throughout the entire abnormal retina, including its proximal portion (Fig. 39L). Pax6 expression pattern did not seem severely altered in the neural retina of mildly affected embryos (Fig. 3-9G), although they displayed unique morphological abnormalities. Lens-specific expression of Pax6 in these embryos appeared confined in the distal lens epithelium (arrowheads in Fig. 3-9G and L) as in GALPR-negative control embryos.

From the whole mount view, the localization of Pax6 staining in the telencephalon and diencephalon did not appear strongly affected (arrows in Fig. 3-9A, F and $\mathrm{K}$ ) although it was expressed at a relatively lower level (compare arrows in Fig. 3-9A and $\mathrm{K}$ ). In addition, the sections of the anterior part of the neural tube continuous with the abnormal retina exhibited dorso-ventrally patterned Pax6 staining (black arrow for dorsal staining and red arrow for ventral in Fig. 3-9H) similar to the distinctive dorsal, middle, and ventral bands of expression seen in the telencephalon of control embryos (black and red arrowheads in Fig. 3-9C). This suggests that the identity of cells localized in specific regions of the brain might be properly maintained in weakly affected embryos despite the fact that the brain is abnormally connected with the morphologically defective retina. 
This brain-specific staining pattern appeared disrupted, with no obvious separation of dorsal and ventral bands in more strongly affected embryos (arrows in Fig. 3-9M). It seems that this alteration in Pax6 expression might not be due to the direct effect of Otx2EnR expression in this region, but rather due to severely disrupted eye morphology consequently preventing the brain from undergoing its proper morphogenesis and thus patterning, as the $R x$ driver does not target the $O t x 2$-EnR transgene in this brain region.

At st 37/38, the heavily pigmented RPE, appearing as a monolayer of cuboidal cells, normally encircles the whole neural retina except the distal most region where the lens is contacting the surface epithelium (Fig. 3-9D and E). At this stage, the control optic cup was clearly separated from the brain even in anterior sections (C) as well as more posterior sections at the level of the lens (D and E). In contrast, in mildly affected embryos, there was the presence of the central lumen abnormally connecting the brain ventricle and the cavity of the optic cup (open arrows in H and I) at anterior levels. At more posterior levels, the lumen appeared no longer contiguous with the optic cup (open arrow in J). As mentioned above, however, posteriorly-bent growing optic vesicle (and the vesicle lumen in it; Arey, 1954; Feduccia and McCrady, 1991) could make the lumen on more posterior sections appear separated from the brain ventricle even though the optic vesicle cavity is still continuous with the brain vesicle. The ventral RPE was often absent in these embryos ( $\mathrm{H}, \mathrm{I}$ and black arrows in $\mathrm{J})$. The boundary between the neural tube and the dorsally localized RPE was obvious by the dark pigmentation of the RPE itself. In severely affected embryos, an enlarged lumen without lateral protrusions was consistently observed (open arrows in $\mathrm{M}$ and $\mathrm{O}$ ), indicating that even the initial outward 
evagination of the neural tube during the formation of the primary optic vesicle is affected in these embryos.

In summary, in severely affected embryos Pax6 expression was altered in several ways, while the localization of Pax6 transcript was properly regionalized to the appropriate layers at stages tested in mildly affected embryos. This proper maintenance of Pax6 expression in the inner neural retina (and its absence in the outer neural retina) during the transition from the optic vesicle to the optic cup suggests that in embryos expressing Otx2-EnR early regionalization of retinal cells into different groups of cells occurs, at least to some extent, despite striking morphological abnormalities in the eye.

Since Pax6 expression in the morphologically abnormal retina of Otx2-EnRexpressing embryos appears grossly similar to wild type at early stages, I next asked whether these Pax6-positive retinal cells in the emerging layers could further differentiate into specific cell types. At stage 41, most of the retinal cells become organized into three retinal laminae: the ganglion cell layer, the inner nuclear layer, and the outer nuclear layer, which contains differentiated photoreceptor cells. Xenopus Pax6 expression is restricted to the ganglion cell layer and the inner part of the inner nuclear layer at this stage (Hirsch and Harris, 1997). The ciliary marginal zone (CMZ) at the extreme periphery of the retina contains undifferentiated mitotic retinoblasts (Wetts et al., 1989). Cells in the CMZ do not express Pax6. Xenopus opsin is expressed in developing photoreceptor cells and is the most abundant protein in differentiated rod cells (Adamus et al., 1991; Knox et al., 1998; Saha and Grainger, 1993; Stiemke et al., 1994). Therefore, the differentiation of all three layers in the mature neural retina can be examined by determining the pattern of Pax6 and opsin expression. Double immunostaining of st 41 
UAS+; GAL- control optic cups using antibodies against PAX6 and rod opsin proteins gave the expected result (top panel in Fig. 3-10). For genotyping, PCR analysis using individual embryos was performed with primers for RFP or GALPR. PAX6 was detected in the nuclei of cells in the ganglion cell layer and the inner nuclear layer, but not in the photoreceptor cell layer. The expression level in the ganglion layer was relatively weaker at this stage (see Fig. 3-10B). PAX6 expression was not detected in the CMZ (outlined in Fig. 3-10B). opsin staining was found in the photoreceptors exclusively. In contrast, PAX6 expression was found in distinctive regions but they were not properly arranged as distinguishable layers in UAS+; GAL+ embryos (E-P), especially in more severely affected embryos (M-P). It is not clear whether these cells indeed represent 'differentiated' retinal neurons normally found in the inner nuclear and/or ganglion cell layers because there were Pax6-positive cells in the morphologically defective retina of Otx2-EnR-expressing embryos even at earlier stages (Fig. 3-9) and they might just continue to express $\operatorname{Pax} 6$ without further differentiation into proper cell types. Markers for specific retinal cell types remain to be tested.

Opsin expression in induced mutant embryos was mainly found in the layer of cells localized in proximity to the pigmented epithelium as in control embryos, consistent with their identity as photoreceptor cells. However, in some cases, opsin staining was abnormally expanded into the thickened RPE layer (O). It is not clear whether some cells in the abnormally wide RPE layer are expressing opsin or cells expressing opsin are located underneath the RPE in the section and the fluorescence signal is bleeded through the superimposed pigmented cells. The most intriguing abnormality found in these embryos is the ectopic opsin expression in a few cells located in the putative ganglion 
cell and the inner nuclear cell layers $(\mathrm{G}, \mathrm{K}, \mathrm{O})$. PAX6 expression was clearly colocalized in, at least some, if not all, of these ectopic opsin-positive cells (arrowheads) as shown in the merged images. This suggests that these cells are not completely converted into photoreceptor cells, retaining some of characteristics of ganglion, interneuron, or some common precursor cells for both cell types. One possible explanation for this ectopic opsin expression is that normally opsin expression is suppressed in cells outside of the photoreceptor layer by Otx2 expression and this suppression is lost due to the Otx2-EnRmediated repression of $O t x 2$. The patchy expression of the Otx2-EnR transgene within the neural retina at this stage explains why only a few scattered cells were affected. Recently Viczian et al. (2003) have shown that Xenopus Otx2 suppressed the expression of an opsin reporter driven by a closely related member of the same family, Otx $5 b$ (Viczian et al., 2003), which is normally expressed in bipolar cells in the inner nuclear cell layer.

These results indicate that in the mature optic cup of embryos expressing Otx2EnR, retinal layering separating the photoreceptor cells from the rest of retinal cells, takes place to some extent, although the precise organization into three different retinal laminae appears disrupted, especially in more severely affected embryos. Existence of more specialized cell types including ganglion cells and various cell types in the inner nuclear layer needs to be tested by cell-type specific molecular markers. Ectopic opsin expression suggests that normal function of Otx2 in the retina may include the repression of the opsin expression outside of the photoreceptor cells, especially in bipolar cells. 


\section{Abnormalities in lens development in Otx2-EnR-expressing embryos}

Vertebrate lens formation occurs progressively through successive phases of bias, specification and determination, each of which is associated with the expression of a number of gene products in the lens ectoderm (Grainger, 1996; Hirsch and Grainger, 2000). It involves the tissue-tissue interactions between the evaginating optic vesicle and the overlying presumptive lens ectoderm (PLE). In Xenopus, Otx2 is expressed in anterior placodal ectoderm at neural plate stage and is one of the earliest genes expressed in the PLE (Zygar et al., 1998). In addition, this gene is also expressed in the developing optic vesicle (Blitz and Cho, 1995; Kablar et al., 1996; Pannese et al., 1995). This expression pattern in both tissues obscures the interpretation of the lens development abnormalities observed in Otx1-/-; Otx2+/- double mutant mice (Martinez-Morales et al., 2001) because it could be due to the role of Otx2 in lens itself or due to its effects in the retina, which can affect the induction of the lens. In our tissue-specific, hormone-inducible system the repression of Otx2 activity occurs in the retina only. Therefore, I was able to examine lens development in a system where Otx2 activity in lens was not affected, but its activity in the retina, the potential lens inducer, was.

The differentiated lens is composed of a layer of proliferating epithelial cells (open arrows in Fig. 3-11A) and elongated, differentiated fiber cells (arrowheads in Fig. 3-11A) that constitute the bulk of the tissue. As new fibers derived from lens epithelium differentiate, their nuclei degenerate (Belecky-Adams et al., 2002; Gilbert, 2000; McDevitt and Brahma, 1973). In the presence of RU486, st 41 UAS+; GALPR+ double transgenic embryos showed apparently abnormal lens, as analyzed by hematoxylin nuclear staining. The lens fiber differentiation was clearly disturbed in these embryos: 
elongated fiber cells were absent and individual nuclear staining was obvious (arrowheads in Fig. 3-11B, C). Note that nuclei of central lens fibers start degenerating at st 40 in $X$. laevis (Nieuwkoop and Faber, 1967). The lens epithelial cells seemed to be present (open arrows in Fig. 3-11B and C). In mildly affected embryos, the disorganized lens was usually reduced in size and mislocated with a rotation toward either dorsal or ventral side. The complete loss of the lens can be observed in most severely affected embryos. These results suggest that repression of Otx 2 activity in the retina is responsible for absence or abnormalities of the lens observed in RU486-treated double transgenic embryos. The precise molecular nature of how Otx2 activity in the retina is linked to the differentiation of the lens remains to be elucidated.

\section{Discussion}

The ability to express a gene product in a temporally and spatially restricted manner is a powerful way of analyzing its role during development. In Xenopus, the most widely used method for functional analysis of a cloned gene is to misexpress or overexpress its wild-type or dominant negative variant mRNA in early embryos. However, the broad ubiquitous or often high level of expression of the gene product by this method can obscure determining the normal spatiotemporal function of the gene. In this Chapter, I describe application of a gene regulatory system that permits control in both time and space of gene construct (see Chapter 2) to an analysis of the function of Otx2 in retinal development in Xenopus tropicalis. 


\section{Primary transgenic approach: its advantages and limits}

Transgenic assays for cis-acting regulatory sequences have been used to identify enhancers in vivo (Kammandel et al., 1999; Knox et al., 1998; Zhang et al., 2003) and present the most reliable way of driving tissue-specific expression. Transgenic frog embryos carrying reporter constructs can be generated quickly in large numbers, which are very useful for subsequent gene expression assays (see pRx-GFP reporter in Chapter 2). However, the disadvantage of a primary transgenic approach for 'gene function' study is that there is variability in the expression level of transgenes, and non-specific background abnormalities associated with the procedure itself. At present our lab obtains a higher fraction of normal transgenic embryos in $X$. laevis. Thus, for these phenotypic analyses, using $X$. laevis embryos rather than $X$. tropicalis would be more efficient at present, although $X$. tropicalis would be more suitable for making stable lines, because of its much shorter life cycle.

To score phenotypes in primary transgenic embryos adequately, it is important to use an approach which allows one to identify transgenic embryos. Because the frequency of obtaining transgenic animals is variable from experiment to experiment, it is useful to be able to exclude the embryos not carrying the transgene for scoring phenotypes. This can be done by simultaneous injection of a second reporter construct. It has been reported that simple mixture of two different constructs resulted in coexpression of both constructs in over $90 \%$ of cases, presumably achieved by co-integration of concatamerized constructs (Hartley et al., 2001; Martynova et al., 2004). Another option is to link the transgenic construct to a second reporter cassette in the same plasmid (Chapter 2). I generated transgenic embryos with a double reporter construct, expressing GFP and RFP 
in two non-overlapping domains; pRx-GFP drives GFP in the eye and pCAR-RFP drives RFP expression in the somites. However, the GFP expression level in the eye of embryos expressing reporters in both domains was found to be greatly reduced consistently in comparison to its expression when pRx-GFP was present by itself (data not shown). Note that same molar amount of DNA was used to make $\mathrm{pRx}-\mathrm{GFP}$ and $\mathrm{pRx}-\mathrm{GFP}-\mathrm{pCAR}-\mathrm{RFP}$ primary transgenic embryos, to eliminate the dose effect on the reporter expression level as the size of the $\mathrm{pRx}-\mathrm{GFP}$ - $\mathrm{pCAR}-\mathrm{RFP}$ plasmid is apparently larger than that of $\mathrm{pRx}-$ GFP. On the other hand, GFP expression in the lens from a plasmid containing both the lens-specific gamma-crystallin promoter driving GFP and pCAR-RFP appeared unaffected (data not shown) although very high level of the gamma-crystallin reporter expression makes it hard to detect a reduction of GFP signal in the eye. This implies the possibility of cross-talk between $R x$ and cardiac actin promoters. Therefore, in experiments to assess the effect of a dominant inhibitory form of $\operatorname{Otx} 2$ on eye development, the pRx-Otx2-EnR construct was utilized alone without being linked to the second reporter, e.g. pCAR-RFP. This resulted in practical limitations in identifying transgenic embryos; PCR analysis had to be carried out on hundreds of embryos to identify those carrying the transgene for the proper scoring of phenotypes. In addition, the results from the PCR analysis suggested the presence of unincorporated plasmids or silent transgene insertions by a potential positional effect, further complicating the phenotypic analysis. These practical limitations motivated me to develop the GAL4/UAS binary gene regulatory system allowing for the establishment of stable transgenic lines to elucidate phenotypes in a more consistent way. 


\section{RU486-mediated inducible system allows rapid induction of transgene expression to} levels capable of efficiently interfering with target gene function.

I demonstrate here that the hormone-dependent GAL4/UAS system can be used to induce the expression of UAS-linked dominant inhibitory gene products in a tissue- and stage-specific fashion. Temporal control was achieved by using RU486 to activate a GAL4 protein containing the progesterone receptor ligand-binding domain. The activation of the UAS-target through this chimeric GAL4 is rapid. The robust expression of the dominant inhibitory form of Otx2, Otx2-EnR, was detected within $3 \mathrm{hr}$ after the administration of RU486, similar to the data from UAS reporters (see Chapter 2). For a dominant negative gene product to repress its target, it must be present when the target gene is expressed in a given tissue. Since the earliest GAL4 transcript expression driven by the $R x$ promoter is detected at the end of gastrulation, fast induction was required for Otx2-EnR expression during early neurula stages. This rapid kinetics of induction would be a desirable goal of any inducible system.

Efficacy of induction of dominant negative construct

RU486 induced temporally controlled expression of Otx2-EnR to levels capable of efficiently interfering with the endogenous Otx2 activity, producing the ocular phenotype. Early eye development appears to be particularly sensitive to changes in levels of OTX2 activity as shown in Otx mutant mice carrying different functional copies of Otx1 and Otx2 (Martinez-Morales et al., 2001). This would explain why Otx2-EnR efficiently produced the ocular phenotypes, as it is difficult, in general, to completely abolish the activity of the target gene product by a dominant negative approach. In 
addition, as discussed in Chapter 2, functional repression of some homeodomain transcription factors including Otx family proteins might not require very high levels of overexpression of dominant-negative gene products (also see below) as suggested by in vitro analysis of Otx orthologs and 'Otx-engrailed' fusion (Furukawa et al., 1997; Li et al., 1999; Singh et al., 1998). Co-injection of a higher dose of Otx2-EnR (at a molar ratio of $7.5: 1$ ), however, was required for the repression of the activation of cement gland markers such as $X A G$ by Otx2 injection in animal cap assays (see Fig. 3-1; Gammill and Sive, 2001) while an equimolar amount of Otx2-EnR failed to efficiently suppress this activation (data not shown). Since I have not tested intermediate doses of Otx2-EnR (i.e., between $1 \times$ and $7.5 \times$ ), it is possible that a dose of Otx2-EnR lower than $7.5 \times$ might be sufficient for the repression. In support of this, Isaacs et al. (1999) have showed that defects in anterior development caused by injection of Otx2-EnR, including the absence of cement gland can be rescued by a similar amount of wild-type Otx2. It also might be speculated that promoter sequences of Otx2 target genes in other tissues including eye could have different binding affinity to OTX2 from those of ectodermal targets involved in cement gland formation and thus one might not need very high dose of Otx2-EnR to antagonize their activity. In addition, GAL4/UAS system is supposed to allow the amplification of the target gene transcription by the potent GAL4 transcriptional activator and multiple copies of its cognate binding site, UAS, combined with our current frog transgenic method permitting high numbers of inserts (e.g. 30-40 copies), for both the UAS effector and GAL4 transgenes. Therefore, it is likely that the gene regulatory system used in this study might have helped to achieve expression level of the Otx2-EnR transgene capable of antagonizing the endogenous OTX2. Whether this system is able to 
direct expression of other dominant inhibitory transgene products at levels that can produce dominant phenotypes might depend on the context of target genes of interest.

Nonetheless, it would be interesting to measure the ratio of expression levels of Otx2-EnR and Otx2 in eye tissue in a quantitative way such as RT-PCR as I have not assayed the precise level of Otx2-EnR expression.

As observed with UAS reporters (Chapter 2), at a dose (25 nM) used for activation, RU486 alone did not affect normal development. No increased lethality was observed with control embryos exposed to RU486 early in development, i.e. from neurula stages to swimming tadpole stages. In addition, the system shows very low background expression of the target gene in the absence of RU486. Only common pattern observed in uninduced embryos is extremely faint expression in the midline. This could be caused by the cardiac actin promoter influencing the transcription of the target gene juxtaposed in the concatamerized transgene constructs.

\section{Spatial control}

Spatial control of the regulatory system was achieved through the well-defined retina-specific $R x$ promoter. The transcription of GALPR RNA recapitulated that of the endogenous $R x$, as revealed by in situ hybridization. The system confers RU486dependent transgene expression only in the target tissue, the developing retina and not in the lens. This is particularly important because cell-autonomous effect of decreased OTX2 activity in the retina must be tested in an experimental system where the lens OTX2 is not affected. Tight spatial control of Otx2-EnR expression is in part demonstrated by the fact that induced mutant embryos showed apparently normal head 
and body axis development and survive to adulthood, unlike Otx2 knockout mice which are headless and die in utero. In addition, this retina-specific GAL4 driver line can be used with potentially many UAS lines bearing different genes implicated in eye development as an invaluable new way to manipulate genes in Xenopus.

While Otx2-EnR effector expression that was indistinguishable from GALPR or GFP expression directed from the same promoter during early optic vesicle stages, it became diffuse and patchy after st 30 . As discussed in detail in Chapter 2, there could be several possible explanations for this. The response of UAS effector gene to the GALPR protein could be variable among cells in the retina, resulting in uneven distribution of the effector expression throughout the retinal tissue. Mechanisms explaining this phenomenon would include a positional effect of the effector transgene integration or the efficiency of RU486 delivery.

Use of inducible system for studies for genes involved in eye development

The gene regulatory system described here provides novel opportunities for the study of genes implicated in eye development. Early pleiotropic effects of many of these 'eye genes' preclude analysis of their later roles since they are often involved in the early development of other tissues including central nervous system (CNS). By using the temporally regulable GAL4 protein, these early effects can be bypassed, permitting the investigation of the effects at desired later stages. In addition, the system permits gene manipulation in each tissue (i.e. lens or retina) using tissue-specific promoters at different developmental stages. Use of this binary regulatory system allowing for control of transgene expression in both stage- and tissue-specific manner also will be highly 
desirable, in general, for studies of other tissue interactions during development. One example is synapse formation at the neuromuscular junction mediated by the interaction of the muscles and neurons, which was studied using a similar GAL4 regulatory system in Drosophila. Osterwalder et al. (2001) were able to change the properties of synapses by hormone-induced pre- or postsynaptic expression of UAS transgenes at the larval neuromuscular junction using tissue-specific promoters. This demonstrates that this system is particularly useful for studying effects of the transgenes in one tissue without affecting the other, or vice versa, when there are potential reciprocal interactions between these two tissues.

\section{Specificity of dominant negative $O t x 2-E n R$ construct.}

High levels of gene activity, especially dominant negative activities, by overexpression often raise the argument that they might interfere with unrelated pathways in addition to the endogenous target (Hemmati-Brivanlou and Melton, 1992; Herskowitz, 1987) and result in non-physiological effects although dominant interfering Otx2-EnR construct used in this study is expressed only in a defined subset of cells (i.e. developing retina) in a more elaborately controlled way using a tissue-specific promoter and a ligand-inducible GAL4. In this section, I will discuss on the specificity of Otx2-EnR construct utilized in this dissertation.

OTX2 protein binds with high affinity to a specific DNA element, TAATCC/T, due to a lysine residue at position 50 of its homeodomain which is very similar to that of the Drosophila transcription factor bicoid (Briata et al., 1999; Hanes and Brent, 1989; Hanes and Brent, 1991; Li et al., 1999; Pannese et al., 1995; Simeone et al., 1993; 
Treisman et al., 1989; Wilson et al., 1993). Therefore, one might expect that normal transcriptional targets of 0 tx 2 would be specifically repressed by activating a fusion protein containing the DNA-binding homeodomain of Otx2 fused to the engrailed repressor domain. This strategy (Conlon et al., 1996; Yang et al., 2002) has been successfully used in Xenopus Otx2 (Gammill and Sive, 2001; Isaacs et al., 1999; Viczian et al., 2003) as well as other members of the Otx family including Crx, a mouse ortholog of Otx5/5b (Furukawa et al., 1997; Germot et al., 2001; Viczian et al., 2003) and SpOtx, a sea urchin homolog of Otx1 and Otx2 (Li et al., 1999). However, it should be mentioned that the phenotypes induced by the expression of the Otx-EnR fusion represent a composite of effects from the repression of a spectrum of Otx2 target genes whose promoters contain Otx2 binding sites (Li et al., 1999). In addition, where Otx2 itself may function as a repressor (Isaacs et al., 1999; Morgan et al., 1999; Nakano et al., 2000); transcription of this class of downstream genes would not be expected to be altered by the Otx2-EnR fusion.

As other bicoid class homeodomain-containing transcription factors and Otx2 homologs show high sequence homology with Otx2 (Germot et al., 2001), especially in their DNA-binding homeodomain, these proteins are hypothesized to share a similar DNA binding specificity with OTX2. In addition, high levels of expression of Otx2-EnR might interfere with non-specific transcriptional targets to which the endogenous OTX2 does not normally bind. Another question is whether replacement of a region carboxylterminal to the homeodomain, which contains a domain important for transcriptional activity of OTX2 protein (Briata et al., 1999; Gammill and Sive, 2001; Nakano et al., 
2000), with the engrailed repressor domain in Otx2-EnR changes binding properties of OTX2. Thus, the specificity of the EnR fusion could be of general concern.

Three lines of evidence support the idea that dominant interfering Otx2-EnR fusion proteins preferentially inhibit OTX2 activity. First, Gammill and Sive (2001) tested the ability of Otx2-EnR to interfere with the activity of siamois (Lemaire et al., 1995), a related paired class homeodomain protein whose expression pattern overlaps with Otx2 during gastrulation stages. In an animal cap assay, the coinjection of siamois and Otx2-engrailed fusion did not affect the induction of genes that siamois alone normally activated.

The homeodomain of another Otx family member, Otx $5 b$ has only one amino acid residue difference from that of $\operatorname{Otx} 2(\operatorname{Ot} x 2=\mathrm{A} ; \operatorname{Ot} x 5 b=\mathrm{S})$. However, lipofection of Xenopus retinal precursors with Otx2-EnR and Otx5b-EnR resulted in different phenotypes: Otx2-EnR-lipofected retina displayed fewer bipolar cells than controls while lipofection of Otx $5 b$-EnR led to decreased number of photoreceptor cells (Viczian et al., 2003). Each cell fate was enhanced by lipofection of wild-type Otx5b and Otx2, respectively. If the engrailed repressor domain contributes to non-specific binding to targets to which Otx2 would normally not bind, then one would expect to see the same lipofection results by both $O t x 2-E n R$ and $O t x 5 b$-EnR. In addition, Isaacs et al. (1999) showed that the phenotype of anterior defects induced by injection of Otx2-EnR mRNA into Xenopus embryos was rescued by coinjection with wild-type Otx2. This suggests that this fusion protein does not act on promoters without Otx2 binding sites. It also would be interesting to test whether the phenotype caused by Otx2-EnR injection is rescued by coinjection of $O t x 5 b$. 
Reciprocal gene replacement experiments in mice suggest at least a partial functional homology between Otx1 and Otx2 genes sharing a high degree of sequence homology (Germot et al., 2001; Kablar et al., 1996), raising a concern that Otx2-EnR might interfere with Otx 1 activity: when the Otx2-coding sequence was replaced by an Otxl-coding cDNA, homozygous mutant mice rescued some of the abnormalities observed in Otx2-/- mice (Acampora et al., 1998). Replacement of Otx1 with Otx2 also produced similar results (Acampora et al., 1999). For Otx2-EnR to inhibit Otx1 activity, however, Otx1 must be present where Otx2-EnR is expressed. Unlike mouse Otx1 and Crx (a mouse counterpart of Otx5/5b), expression patterns of Xenopus cognates of these genes, Otx1 and Otx5b (and Otx4; Kablar et al., 1996; Viczian et al., 2003), respectively, do not appear to overlap with that of Otx2 or Otx2-EnR in the eye primordia at late neurula and early tail bud stages when the Otx2-EnR is believed to actively inhibit the OTX2 activity (see the text). Nonetheless, in order to confirm the specificity of Otx2EnR, control experiments remain to be performed using closely related Xenopus Otx1 or Otx $5 b$ either by animal cap assay or phenotypic rescue by co-injection of RNAs.

\section{References}

\section{Acampora, D., Avantaggiato, V., Tuorto, F., Barone, P., Perera, M., Choo, D., Wu,} D., Corte, G. and Simeone, A. (1999). Differential transcriptional control as the major molecular event in generating Otx1-/- and Otx2-/- divergent phenotypes. Development 126, $1417-26$. 
Acampora, D., Avantaggiato, V., Tuorto, F., Briata, P., Corte, G. and Simeone, A. (1998). Visceral endoderm-restricted translation of Otx1 mediates recovery of Otx2 requirements for specification of anterior neural plate and normal gastrulation. Development 125, 5091-104.

Acampora, D., Gulisano, M., Broccoli, V. and Simeone, A. (2001). Otx genes in brain morphogenesis. Prog Neurobiol 64, 69-95.

Acampora, D., Mazan, S., Lallemand, Y., Avantaggiato, V., Maury, M., Simeone, A. and Brulet, P. (1995). Forebrain and midbrain regions are deleted in Otx2-/- mutants due to a defective anterior neuroectoderm specification during gastrulation. Development 121, 3279-90.

Adamus, G., Zam, Z. S., Arendt, A., Palczewski, K., McDowell, J. H. and Hargrave, P. A. (1991). Anti-rhodopsin monoclonal antibodies of defined specificity: characterization and application. Vision Research 31, 17-31.

Andreazzoli, M., Gestri, G., Angeloni, D., Menna, E. and Barsacchi, G. (1999). Role of Xrx1 in Xenopus eye and anterior brain development. Development 126, 2451-60. Ang, S. L., Jin, O., Rhinn, M., Daigle, N., Stevenson, L. and Rossant, J. (1996). A targeted mouse Otx2 mutation leads to severe defects in gastrulation and formation of axial mesoderm and to deletion of rostral brain. Development 122, 243-52.

Arey, L. B. (1954). Developmental anatomy. Philadelphia and London: W. B. Saunders Company.

Ashery-Padan, R., Marquardt, T., Zhou, X. and Gruss, P. (2000). Pax6 activity in the lens primordium is required for lens formation and for correct placement of a single retina in the eye. Genes Dev 14, 2701-11. 
Baas, D., Bumsted, K. M., Martinez, J. A., Vaccarino, F. M., Wikler, K. C. and Barnstable, C. J. (2000). The subcellular localization of Otx2 is cell-type specific and developmentally regulated in the mouse retina. Brain Res Mol Brain Res 78, 26-37.

Belecky-Adams, T. L., Adler, R. and Beebe, D. C. (2002). Bone morphogenetic protein signaling and the initiation of lens fiber cell differentiation. Development 129, 3795-802.

Blitz, I. L. and Cho, K. W. (1995). Anterior neurectoderm is progressively induced during gastrulation: the role of the Xenopus homeobox gene orthodenticle. Development Supplement 121, 993-1004.

Bovolenta, P., Mallamaci, A., Briata, P., Corte, G. and Boncinelli, E. (1997).

Implication of OTX2 in pigment epithelium determination and neural retina differentiation. J Neurosci 17, 4243-52.

Briata, P., Ilengo, C., Bobola, N. and Corte, G. (1999). Binding properties of the human homeodomain protein OTX2 to a DNA target sequence. FEBS Lett 445, 160-4.

Bronchain, O. J., Hartley, K. O. and Amaya, E. (1999). A gene trap approach in Xenopus. Current Biology 9, 1195-1198.

Brun, R. B. (1981). The movement of the prospective eye vesicles from the neural plate into the neural fold in Ambystoma mexicanum and Xenopus laevis. Developmental Biology 88, 192-9.

Casarosa, S., Andreazzoli, M., Simeone, A. and Barsacchi, G. (1997). Xrx1, a novel Xenopus homeobox gene expressed during eye and pineal gland development. Mech Dev 61, 187-98.

Chow, R. L. and Lang, R. A. (2001). Early eye development in vertebrates. Annu Rev Cell Dev Biol 17, 255-96. 
Conlon, F. L., Sedgwick, S. G., Weston, K. M. and Smith, J. C. (1996). Inhibition of Xbra transcription activation causes defects in mesodermal patterning and reveals autoregulation of Xbra in dorsal mesoderm. Development 122, 2427-35.

Eagleson, G., Ferreiro, B. and Harris, W. A. (1995). Fate of the anterior neural ridge and the morphogenesis of the Xenopus forebrain. Journal of Neurobiology 28, 146-158.

Eagleson, G. W. and Harris, W. A. (1990). Mapping of the Presumptive Brain Regions in the Neural Plate of Xenopus Laevis. Journal of Neurobiology 21, 427-440.

Feduccia, A. and McCrady, E. (1991). Torrey's morphogenesis of the vertebrates. New York: John Wiley \& Sons, Inc.

Finkelstein, R., Smouse, D., Capaci, T. M., Spradling, A. C. and Perrimon, N. (1990). The orthodenticle gene encodes a novel homeo domain protein involved in the development of the Drosophila nervous system and ocellar visual structures. Genes Dev 4, 1516-27.

Furukawa, T., Morrow, E. M. and Cepko, C. L. (1997). Crx, a novel otx-like homeobox gene, shows photoreceptor-specific expression and regulates photoreceptor differentiation. Cell 91, 531-41.

Furuta, Y. and Hogan, B. L. (1998). BMP4 is essential for lens induction in the mouse embryo. Genes Dev 12, 3764-75.

Gammill, L. S. and Sive, H. (1997). Identification of otx2 target genes and restrictions in ectodermal competence during Xenopus cement gland formation. Development 124, 471-81. 
Gammill, L. S. and Sive, H. (2001). otx2 expression in the ectoderm activates anterior neural determination and is required for Xenopus cement gland formation.

Developmental Biology 240, 223-36.

Germot, A., Lecointre, G., Plouhinec, J. L., Le Mentec, C., Girardot, F. and Mazan, S. (2001). Structural evolution of Otx genes in craniates. Mol Biol Evol 18, 1668-78.

Gilbert, S. F. (2000). Developmental Biology. Sunderland: Sinauer Associates, Inc. Grainger, R. M. (1996). New perspectives on embryonic lens induction. Seminars in Cell and Developmental Biology 7, 149-55.

Grant, P. and Rubin, E. (1980). Ontogeny of the retina and optic nerve in Xenopus laevis. II. Ontogeny of the optic fiber pattern in the retina. J Comp Neurol 189, 671-98. Green, M. R., Maniatis, T. and Melton, D. A. (1983). Human beta-globin pre-mRNA synthesized in vitro is accurately spliced in Xenopus oocyte nuclei. Cell 32, 681-94. Hanes, S. D. and Brent, R. (1989). DNA specificity of the bicoid activator protein is determined by homeodomain recognition helix residue 9. Cell 57, 1275-83.

Hanes, S. D. and Brent, R. (1991). A genetic model for interaction of the homeodomain recognition helix with DNA. Science 251, 426-30.

Harland, R. M. (1991). In situ hybridization: an improved whole-mount method for Xenopus embryos. Methods in Cell Biology 36, 685-695.

Hartley, K. O., Hardcastle, Z., Friday, R. V., Amaya, E. and Papalopulu, N. (2001). Transgenic Xenopus embryos reveal that anterior neural development requires continued suppression of BMP signaling after gastrulation. Developmental Biology 238, 168-84. 
Hemmati-Brivanlou, A. and Melton, D. A. (1992). A truncated activin receptor inhibits mesoderm induction and formation of axial structures in Xenopus embryos. Nature 359, 609-14.

Herskowitz, I. (1987). Functional inactivation of genes by dominant negative mutations. Nature 329, 219-222.

Hide, T., Hatakeyama, J., Kimura-Yoshida, C., Tian, E., Takeda, N., Ushio, Y., Shiroishi, T., Aizawa, S. and Matsuo, I. (2002). Genetic modifiers of otocephalic phenotypes in Otx2 heterozygous mutant mice. Development 129, 4347-57.

Hirsch, N. and Grainger, R. M. (2000). Induction of the lens. Results \& Problems in Cell Differentiation 31, 51-68.

Hirsch, N. and Harris, W. A. (1997). Xenopus Pax-6 and retinal development. Journal of Neurobiology 32, 45-61.

Hirsch, N., Zimmerman, L. B., Gray, J., Chae, J., Curran, K. L., Fisher, M., Ogino, H. and Grainger, R. M. (2002). Xenopus tropicalis transgenic lines and their use in the study of embryonic induction. Developmental Dynamics 225, 522-35.

Hollyfield, J. G. (1973). Elimination of egg pigment from developing ocular tissues in the frog Rana pipiens. Developmental Biology 30, 115-28.

Holt, C. E. (1980). Cell movements in Xenopus eye development. Nature 287, 850-852. Isaacs, H. V., Andreazzoli, M. and Slack, J. M. (1999). Anteroposterior patterning by mutual repression of orthodenticle and caudal-type transcription factors. Evol Dev 1, 14352. 
Kablar, B., Vignali, R., Menotti, L., Pannese, M., Andreazzoli, M., Polo, C., Giribaldi, M. G., Boncinelli, E. and Barsacchi, G. (1996). Xotx genes in the developing brain of Xenopus laevis. Mech Dev 55, 145-58.

Kammandel, B., Chowdhury, K., Stoykova, A., Aparicio, S., Brenner, S. and Gruss, P. (1999). Distinct cis-essential modules direct the time-space pattern of the Pax6 gene activity. Developmental Biology 205, 79-97.

Knox, B. E., Schlueter, C., Sanger, B. M., Green, C. B. and Besharse, J. C. (1998). Transgene expression in Xenopus rods. FEBS Letters 423, 117-21.

Krieg, P. A., Varnum, S. M., Wormington, W. M. and Melton, D. A. (1989). The mRNA encoding elongation factor 1-alpha (EF-1 alpha) is a major transcript at the midblastula transition in Xenopus. Developmental Biology 133, 93-100.

Lemaire, P., Garrett, N. and Gurdon, J. B. (1995). Expression cloning of Siamois, a Xenopus homeobox gene expressed in dorsal-vegetal cells of blastulae and able to induce a complete secondary axis. Cell $\mathbf{8 1}, 85-94$.

Li, X., Wikramanayake, A. H. and Klein, W. H. (1999). Requirement of SpOtx in cell fate decisions in the sea urchin embryo and possible role as a mediator of beta-catenin signaling. Developmental Biology 212, 425-39.

Li, Z., Joseph, N. M. and Easter, S. S., Jr. (2000). The morphogenesis of the zebrafish eye, including a fate map of the optic vesicle. Dev Dyn 218, 175-88.

Lopashov, G. V. and Stroeva, O. G. (1964). Development of the eye. Experimental studies. Jerusalem: Israel Program for Scientific Translations Ltd. 
Mallamaci, A., Di Blas, E., Briata, P., Boncinelli, E. and Corte, G. (1996). OTX2 homeoprotein in the developing central nervous system and migratory cells of the olfactory area. Mech Dev 58, 165-78.

Martinez-Morales, J. R., Rodrigo, I. and Bovolenta, P. (2004). Eye development: a view from the retina pigmented epithelium. Bioessays 26, 766-77.

Martinez-Morales, J. R., Signore, M., Acampora, D., Simeone, A. and Bovolenta, P. (2001). Otx genes are required for tissue specification in the developing eye.

Development 128, 2019-30.

Martynova, N., Eroshkin, F., Ermakova, G., Bayramov, A., Gray, J., Grainger, R. and Zaraisky, A. (2004). Patterning the forebrain: FoxA4a/Pintallavis and Xvent2 determine the posterior limit of Xanf1 expression in the neural plate. Development 131, 2329-38.

Matsuda, M. and Keino, H. (2001). Possible roles of beta-catenin in evagination of the optic primordium in rat embryos. Dev Growth Differ 43, 391-400.

Matsuo, I., Kuratani, S., Kimura, C., Takeda, N. and Aizawa, S. (1995). Mouse Otx2 functions in the formation and patterning of rostral head. Genes Dev 9, 2646-58.

Mayor, R., Morgan, R. and Sargent, M. G. (1995). Induction of the prospective neural crest of Xenopus. Development 121, 767-77.

McDevitt, D. S. and Brahma, S. K. (1973). Ontogeny and localization of the crystallins during embryonic lens development in Xenopus laevis. J Exp Zool 186, 127-40.

Morgan, R., Hooiveld, M. H., In der Reiden, P. and Durston, A. J. (1999). A conserved 30 base pair element in the Wnt-5a promoter is sufficient both to drive its' early embryonic expression and to mediate its' repression by otx2. Mech Dev 85, 97-102. 
Nakano, T., Murata, T., Matsuo, I. and Aizawa, S. (2000). OTX2 directly interacts with LIM1 and HNF-3beta. Biochem Biophys Res Commun 267, 64-70.

Nieuwkoop, P. D. (1963). Pattern formation in artificially activated ectoderm (Rana pipiens and Ambystoma punctatum). Developmental Biology 7, 255-79.

Nieuwkoop, P. D. and Faber, J. (1967). Normal table of Xenopus laevis. Amsterdam: North Holland Publishing.

Nishida, A., Furukawa, A., Koike, C., Tano, Y., Aizawa, S., Matsuo, I. and Furukawa, T. (2003). Otx2 homeobox gene controls retinal photoreceptor cell fate and pineal gland development. Nat Neurosci 6, 1255-63.

Offield, M. F., Hirsch, N. and Grainger, R. M. (2000). The development of Xenopus tropicalis transgenic lines and their use in studying lens developmental timing in living embryos. Development 127, 1789-97.

Pannese, M., Polo, C., Andreazzoli, M., Vignali, R., Kablar, B., Barsacchi, G. and Boncinelli, E. (1995). The Xenopus homologue of Otx2 is a maternal homeobox gene that demarcates and specifies anterior body regions. Development 121, 707-20.

Perron, M., Kanekar, S., Vetter, M. L. and Harris, W. A. (1998). The genetic sequence of retinal development in the ciliary margin of the Xenopus eye. Developmental Biology 199, 185-200.

Rhinn, M., Dierich, A., Shawlot, W., Behringer, R. R., Le Meur, M. and Ang, S. L. (1998). Sequential roles for Otx2 in visceral endoderm and neuroectoderm for forebrain and midbrain induction and specification. Development 125, 845-56. 
Saha, M. S. and Grainger, R. M. (1993). Early opsin expression in Xenopus embryos precedes photoreceptor differentiation. Brain Research. Molecular Brain Research 17, 307-18.

Schedl, A., Ross, A., Lee, M., Engelkamp, D., Rashbass, P., van Heyningen, V. and Hastie, N. D. (1996). Influence of PAX6 gene dosage on development: overexpression causes severe eye abnormalities. Cell 86, 71-82.

Schook, P. (1980a). Morphogenetic movements during the early development of the chick eye. A light microscopic and spatial reconstructive study. Acta Morphol Neerl Scand 18, 1-30.

Schook, P. (1980b). Morphogenetic movements during the early development of the chick eye. An ultrastructural and spatial reconstructive study. A. Invagination of the lens placode. Acta Morphol Neerl Scand 18, 133-57.

Schook, P. (1980c). Morphogenetic movements during the early development of the chick eye. An ultrastructural and spatial reconstructive study. B. Invagination of the optic vesicle and fusion of its walls. Acta Morphol Neerl Scand 18, 159-80.

Simeone, A., Acampora, D., Mallamaci, A., Stornaiuolo, A., MR, D. A., Nigro, V. and Boncinelli, E. (1993). A vertebrate gene related to orthodenticle contains a homeodomain of the bicoid class and demarcates anterior neuroectoderm in the gastrulating mouse embryo. EMBO Journal 12, 2735-47.

Simeone, A., Puelles, E. and Acampora, D. (2002). The Otx family. Curr Opin Genet Dev 12, 409-15. 
Singh, S., Tang, H. K., Lee, J. Y. and Saunders, G. F. (1998). Truncation mutations in the transactivation region of PAX6 result in dominant-negative mutants. $J$ Biol Chem 273, 21531-41.

Sive, H. L., Grainger, R. M. and Harland, R. M. (2000). Early development of Xenopus laevis. A laboratory manual. New York: Cold Spring Harbor Laboratory Press. Stiemke, M. M., Landers, R. A., al-Ubaidi, M. R., Rayborn, M. E. and Hollyfield, J. G. (1994). Photoreceptor outer segment development in Xenopus laevis: influence of the pigment epithelium. Developmental Biology 162, 169-80.

Svoboda, K. K. and O'Shea, K. S. (1987). An analysis of cell shape and the neuroepithelial basal lamina during optic vesicle formation in the mouse embryo. Development 100, 185-200.

Treisman, J., Gonczy, P., Vashishtha, M., Harris, E. and Desplan, C. (1989). A single amino acid can determine the DNA binding specificity of homeodomain proteins. Cell 59, 553-62.

Viczian, A. S., Vignali, R., Zuber, M. E., Barsacchi, G. and Harris, W. A. (2003). XOtx $5 \mathrm{~b}$ and XOtx2 regulate photoreceptor and bipolar fates in the Xenopus retina. Development 130, 1281-94.

Wetts, R., Serbedzija, G. and Fraser, S. E. (1989). Cell Lineage Analysis Reveals Multipotent Precursors in the Ciliary Margin of the Frog Retina. Developmental Biology 136, 254-263.

Wilson, D., Sheng, G., Lecuit, T., Dostatni, N. and Desplan, C. (1993). Cooperative dimerization of paired class homeo domains on DNA. Genes Dev 7, 2120-34. 
Yang, H., Lu, M. M., Zhang, L., Whitsett, J. A. and Morrisey, E. E. (2002). GATA6 regulates differentiation of distal lung epithelium. Development 129, 2233-46. Zhang, L., El-Hodiri, H. M., Ma, H. F., Zhang, X., Servetnick, M., Wensel, T. G. and Jamrich, M. (2003). Targeted expression of the dominant-negative FGFR4a in the eye using Xrx1A regulatory sequences interferes with normal retinal development. Development 130, 4177-86.

Zuber, M. E., Gestri, G., Viczian, A. S., Barsacchi, G. and Harris, W. A. (2003). Specification of the vertebrate eye by a network of eye field transcription factors. Development 130, 5155-67.

Zygar, C. A., Cook, T. L. and Grainger, R. M., Jr. (1998). Gene activation during early stages of lens induction in Xenopus. Development 125, 3509-19. 
Figure 3-1. Dominant inhibitory Otx2-EnR represses Otx2 activity in animal cap assay (after Gammill and Sive, 2001). (A) Schematic diagram showing the domain structure of wild-type Otx2 (top) and Otx2-EnR fusion (bottom). The $3^{\prime}$ region of Otx2 including the activation domain (AD) is replaced with the Drosophila engrailed repressor domain (EnR) to generate Otx2-EnR. HD indicates homeodomain. (B) Both blastomeres of a two cell-stage embryo were microinjected with $O t x 2$ with or without Otx2-EnR mRNA. Animal caps were dissected at stage 9 and harvested for RT-PCR at stage 15. (C) Animal caps isolated from embryos injected with following RNAs (lane 1, 500 pg GFP; lane 2, 50 pg Otx2 plus 500 pg GFP; lane 3, 50 pg Otx2 plus 50 pg of Otx2-EnR plus 500 pg GFP; lane 4, 50 pg Otx2 plus 500 pg Otx2-EnR) were assayed by RT-PCR for expression of the cement gland marker $X A G-1$. The amount of injected RNA was normalized using GFP mRNA. EF-1 $\alpha$ was used as a loading control. 
A

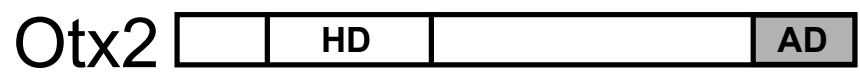

\begin{tabular}{l|l|l|l|}
\hline Otx2-EnR & HD & & EnR \\
\hline
\end{tabular}

B

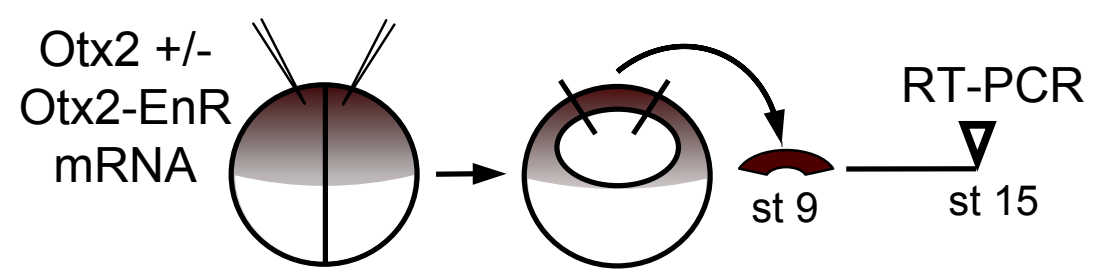

C

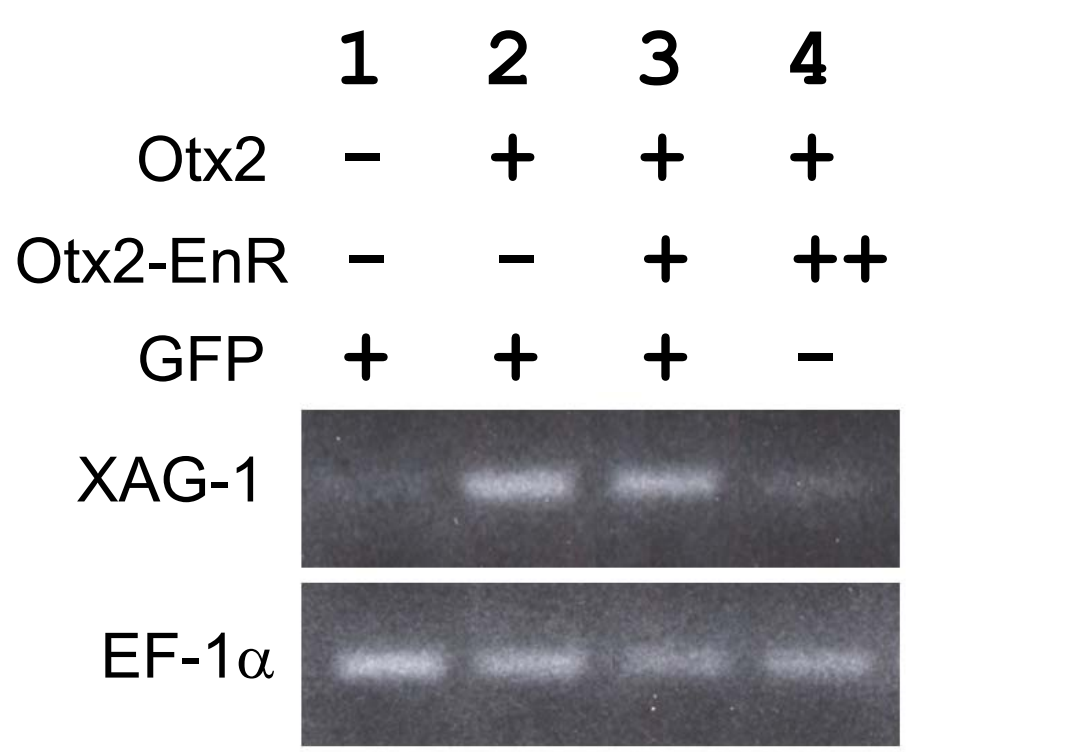


Figure 3-2. Retina-specific inhibition of Otx2 activity by primary transgenic strategy causes ocular defects. (A) A diagram of the pRx-Otx2-EnR construct used to generate the primary transgenic embryos. The $\mathrm{pRx}-$ Otx2-EnR plasmid contains the Xenopus $R x$ promoter (pRx) driving the Otx2 homeodomain (HD) fused to the Drosophila engrailed repressor domain (EnR). Arrows indicate the direction of PCR primers used in genomic PCR analysis to identify embryos carrying the transgene. Sense and antisense primers are derived from the regions corresponding to the $R x$ promoter and the $\operatorname{Otx} 2$ homeodomain, respectively, amplifying only the transgene, not the endogenous $R x$ or Otx2 locus. (B) Dorsal view image of representative transgenic embryos at stage 41/42 generated with pRx-Otx2-EnR, displaying normal (a), mild (b and c) or severe (d) eye phenotype including extended RPE connected to the eye (arrow). (C) A table comparing transgenic embryos generated with pRx-Otx2-EnR with those containing the control construct, $\mathrm{pRx}-$ GFP. As in (B), the percentage of embryos with normal (a), mild (b and c) or severe (d) eye phenotype is shown here. Each percentage value is noted within parentheses next to actual numbers of embryos. (e) The percentage of embryos displaying the severe gastrulation/anterior defects that were not scored as eye defects. 


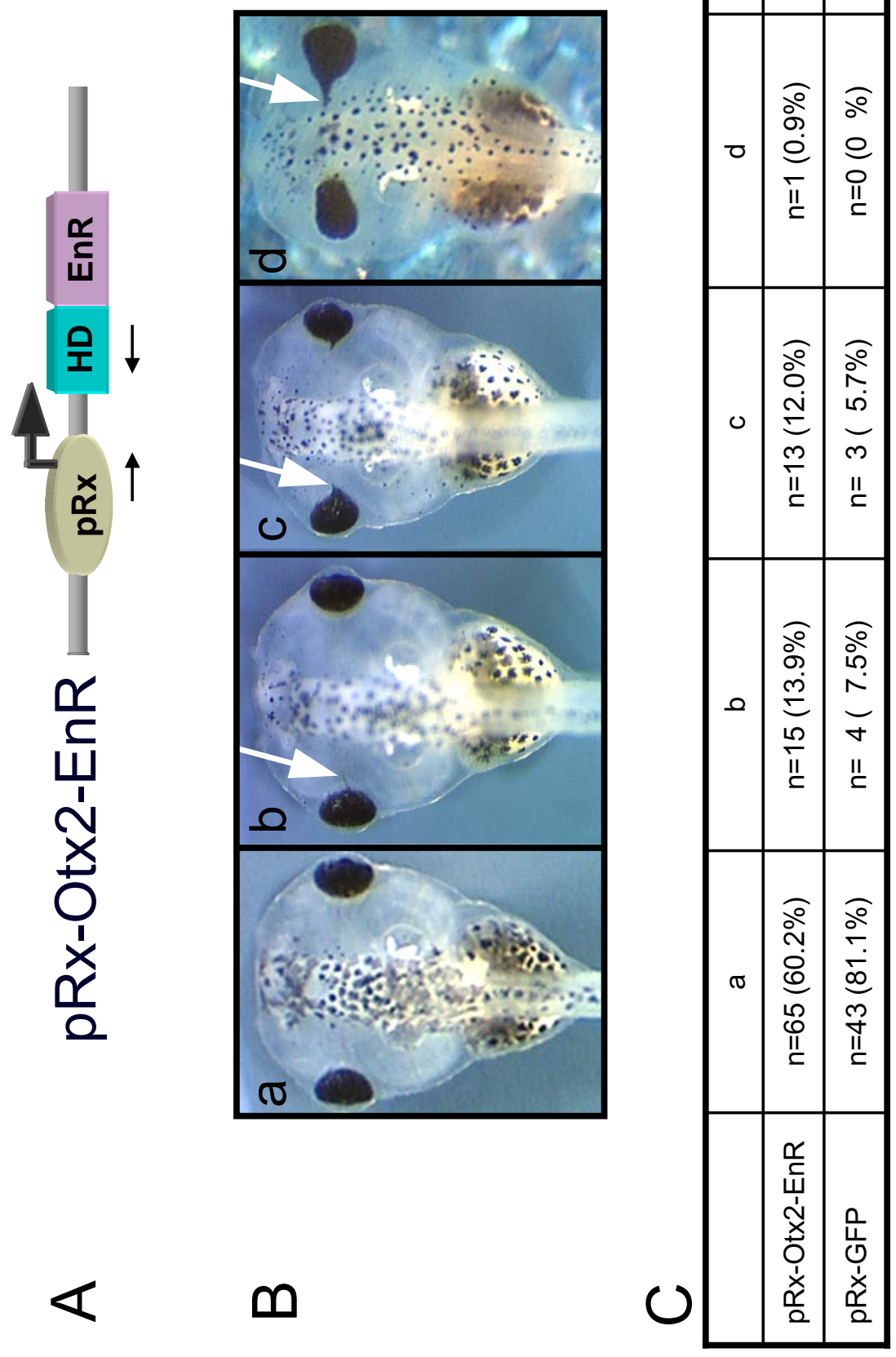


Figure 3-3. RU486-induced expression of a dominant negative variant of Otx2 within eye tissue produces ocular defects. (A) A pRx-GALPR line driving a hormone-inducible transactivator in the retina was crossed to a UAS-Otx2-EnR line which carries a UASdriven chimeric construct, Otx2-EnR, the Otx2 homeodomain fused to the Drosophila engrailed repressor domain (EnR), linked to a pCAR-RFP cassette. In the double transgenic progeny, chimeric GAL4 (gray ovals) is posttranslationally activated by addition of RU486 (blue circles), thereby inducing transcription from UAS-Otx2-EnR. The dominant inhibitory Otx2-EnR then presumably represses the endogenous OTX2 transcriptional activity in retina. Embryos from this cross were treated with 0 (solvent control) or $25 \mathrm{nM}$ RU486 from stage 11/12 onwards for 48 or $72 \mathrm{hr}$ until they reached st 37 (E) or st 41 (B-D, F-I), respectively. (B) Lateral views of st 41 embryos from the cross described in A. As indicated on the top, eye abnormalities (arrows) were observed only in RU486-treated (25 nM) embryos carrying the UAS target gene (UAS+, i.e. RFP-positive) and not in untreated ( $0 \mathrm{nM})$ UAS+ or UAS- embryos. RU486-treated, but RFP-negative (i.e. UAS-) embryos showed no eye phenotype (data not shown). (C) RFP image of embryos in B. Somitic muscle-specific RFP expression driven by the pCAR-RFP is apparent in UAS+ embryos carrying the UAS-Otx2-EnR--pCAR-RFP transgene, but not in UAS- embryos. (D) Enlarged view of $25 \mathrm{nM}$ RU486-treated embryos in the dotted box in B. Severely affected embryos (marked by **) show abnormal eyes (arrowheads) greatly reduced in size while the mildly affected embryos (*) display an incomplete closure of the optic fissure (arrows) of almost normal size eye. (E) St 37 embryos cultured in solvent only $(0 \mathrm{nM})$ or $25 \mathrm{nM}$ RU486. Only RU486-treated embryos presented a typical eye phenotype of widely open optic fissures (arrows). (F-H) Dorsal 
view of embryos in D, treated with $25 \mathrm{nM}$ RU486 $(\mathrm{G}, \mathrm{H})$ or untreated control embryos (F). (F) Only a thin optic nerve (arrow; invisible here) is present between the eye and the brain of the untreated embryos. (G) Severely affected embryos display a morphologically abnormal eye cup (arrow) which is directly connected with the brain. (H) Narrow RPE tissue (arrow) extended from a relatively normal optic cup is connected with the neural tube in weakly affected embryos. (I) Head to head comparison of embryos in F (0 nM) and $\mathrm{G}(25 \mathrm{nM})$. The width of the head region encompassing the eye was significantly reduced in RU486-treated embryos while the width of the brain itself looks comparable to that of untreated embryos. 

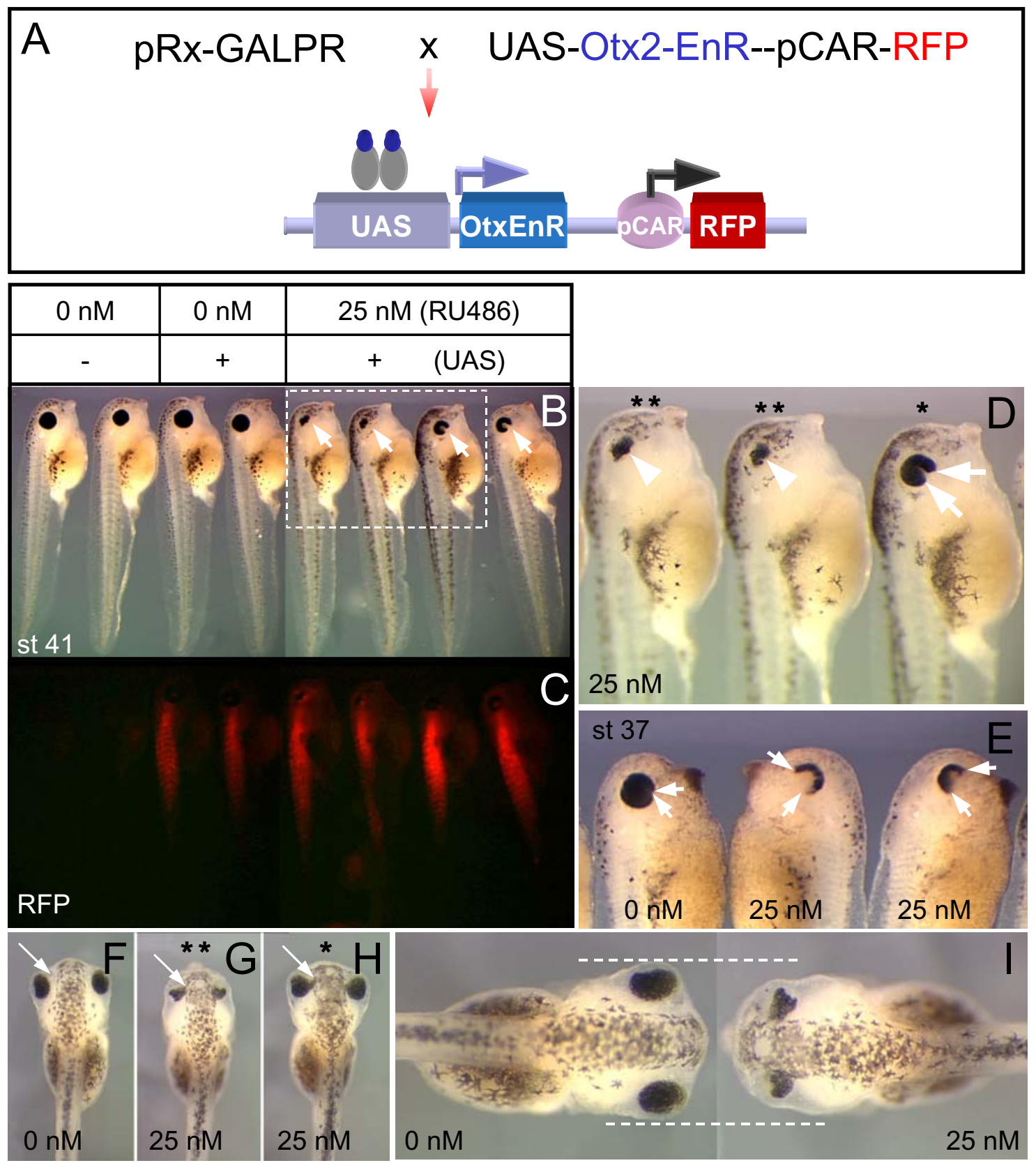
Table 3-1. Defects in eye morphology in the progeny of the cross of pRx-GALPR and UAS-Otx2-EnR animals ${ }^{\mathrm{a}}$

\begin{tabular}{rcccc}
\hline RU486 & RFP exp. & Normal & Abnormal & Total \\
\hline $0 \mathrm{nM}$ & RFP- & 106 & 0 & 106 \\
$0 \mathrm{nM}$ & RFP+ & 99 & $2^{\mathrm{b}}$ & 101 \\
$25 \mathrm{nM}$ & RFP- & 105 & 0 & 105 \\
$25 \mathrm{nM}$ & RFP+ & 59 & $40(* * 24+* 16)$ & 99 \\
\hline
\end{tabular}

a Embryos from the cross of the pRx-GALPR transactivator line with the UAS-Otx2-EnR effector line were incubated with RU486 for $72 \mathrm{~h}$ from stage 11/12 onward and examined for morphological abnormalities in the eye. Each of approximately 100 embryos either RFP-negative (RFP-) or RFP-positive (RFP+; i.e. carrying the effector gene) cultured in 0 (solvent control) or $25 \mathrm{nM}$ of RU486, respectively, was scored as strongly defective $(* *)$, mildly defective $\left.{ }^{*}\right)$, or normal eye phenotype (also see Fig. 3-3). Note that the fraction of RFP+ embryos expected to carry both activator and effector transgenes, displaying the abnormal phenotype, is a maximum of $50 \%$, and 40 out of 99 RU486-treated RFP+ embryos showed the phenotype in this experiment. The statistical significance was determined by a $\chi^{2}$ test, and the probability $(p>0.05)$ is great enough to accept the hypothesis that obtained numbers represent the expected $50 \%$. The same assay was performed with embryos cultured in RU486 for $48 \mathrm{~h}$ and similar results were obtained (data not shown). ${ }^{\mathrm{b}}$ Embryos displayed a very weak eye phenotype that could be explained by non-specific developmental abnormalities. 
Figure 3-4. Histological analyses of embryos and adult Xenopus tropicalis expressing Otx2-EnR. Animals shown here are from the cross described in Fig. 3-3. Embryos were cultured in the presence of $25 \mathrm{nM}$ RU486 until st 41, and then transferred to and raised in normal culture media without RU486 until indicated stages. (A-E, G-I) Images of transverse sections through the eye of control sibling and Otx2-EnR-expressing embryos at indicated stages. (A) Side-by-side comparison of the left and right eye vesicles from one individual embryo at st 22/23 hybridized with a probe for EnR. While the optic vesicle on one side (red open arrow) appears normal, the other side displays the morphologically abnormal vesicle (black open arrow). Note that the optic vesicle is normally continuous with the neural tube at this stage. (B) St 29 optic vesicle of Otx2EnR-negative control embryo in transition to forming an optic cup. Arrow indicates a thin layer of presumptive pigmented epithelium attached to the neural tube only by the narrow stalk (arrowheads) at the ventral pole. Open arrow points out the vesicle cavity clearly separated from the lumen $(*)$ of the brain. Thickening of the lens ectoderm (open arrowhead) is apparent. Staining in the retina represents the endogenous Otx2 expression. (C) Abnormal eye vesicle of a st 29 embryo expressing Otx2-EnR as detected by in situ hybridization using a probe for EnR (blue staining in the retina). The vesicle epithelium (arrow) is abnormally connected with the neural tube and the lumen (*) is broadly continuous with the vesicle cavity (open arrow). A small cavity (red open arrow) which is separated from the brain lumen is also shown here. (D) An Otx2-EnR-negative st 37 embryo displaying the well-formed optic cup completely separated from the brain. Dorsal (arrow) and ventral (arrowhead) RPE surrounds the whole neural retina. (E) The optic cup of Otx2-EnR-positive (purple staining within the retina) embryos is continuous with 
the forebrain through a wide central lumen (open arrows) and lacks ventral RPE (*). (G) St 41 normal optic cup of control GALPR- embryo showing RPE formed both dorsally (arrow) and ventrally (arrowhead). (H, I) St 41 defective optic cup directly connected with the brain through a wide RPE-free region (open arrows) in UAS+; GALPR+ mildly affected $(\mathrm{H})$ and severely affected (I) embryos. Arrows indicate abnormally thick dorsal RPE while arrowheads indicate ventral RPE. (I) The * indicates almost complete loss of ventral RPE except a small patch of pigmented cells (arrowhead). (F) Three-week-old tadpole showing the heavily pigmented eye (arrow) directly attached to lateral part of the forebrain which is also pigmented (arrowhead). The other eye (open arrow) is well separated from the brain although the optic fissure is wide open (data not shown). (J-L) External phenotype in froglets after metamorphosis. (J) Normal external eye with properly developed iris (arrow) in a control sibling. (K) Relatively mild phenotype consists of a pigmented protrusion (arrow). (L) Arrow points to the small impression representing the most severely affected eye. E, ear; le, lens; N, nose; nt, neural tube. 


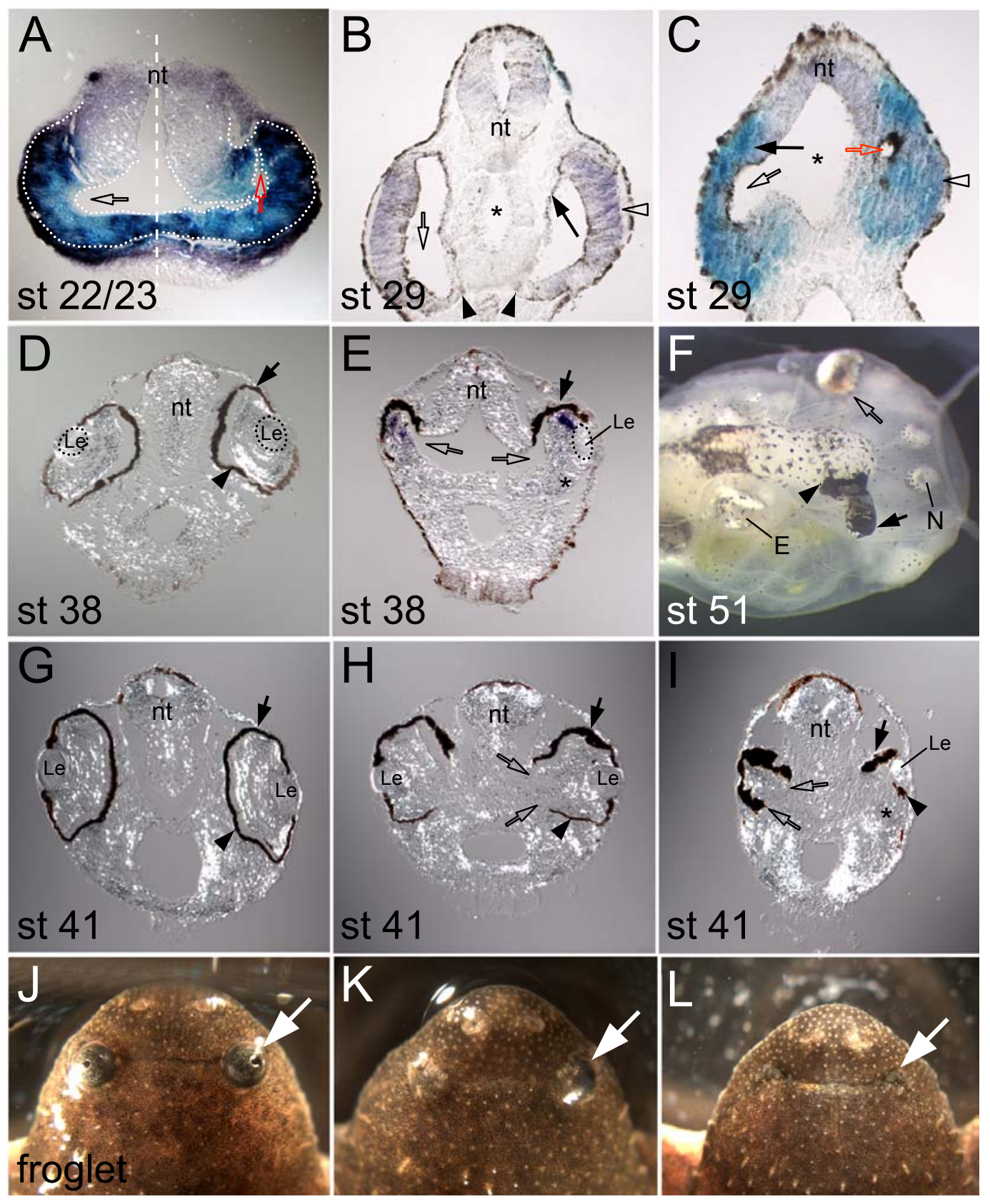


Figure 3-5. Rapid induction of Otx2-EnR in the retina by RU486. (A-F) Embryos from the cross described in Fig. 3-3 were cultured in the presence of RU486 from st 11/12 for the indicated number of hours and then analyzed by in situ hybridization with a probe for the EnR domain. Note that the staining is mainly confined to the presumptive retinal field throughout the stages examined. Arrows in $\mathrm{E}$ and $\mathrm{F}$ indicate punctate clusters of cells showing expression within the retina. 


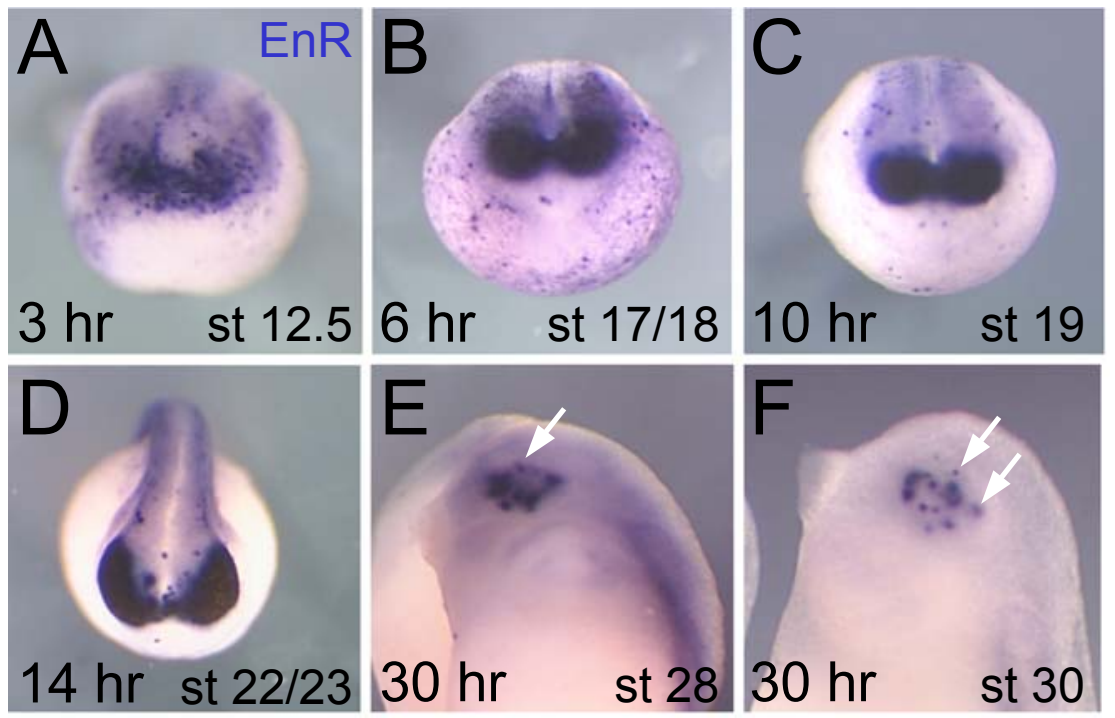


Figure 3-6. Expression of native Otx2 and $R x$ promoter-driven Otx2-EnR overlap within the presumptive retina. Double in situ hybridization performed on stage 19 embryos (A, A', B, B'; with EnR and $R x$ probes) or $22 / 23$ embryos (C, C', D, D'; with EnR and Otx2 probes) from the cross described in Fig. 3-3. Embryos were cohybridized with both probes and visualization of each probe was carried out sequentially. Otx2-EnR expression was first revealed by light blue staining (A-D) followed by the detection of Otx2 or Rx RNA by dark purple staining (A'-D'). Note that Otx2-EnR expression (arrows in A and C) is overlapping with $R x\left(\mathrm{~A}^{\prime}\right)$ or $\operatorname{Otx} 2\left(\mathrm{C}^{\prime}\right)$ in the presumptive retina of the same embryos. The staining patterns of $R x$ or Otx2 in the Otx2-EnR-expressing embryos should be compared with those in the Otx2-EnR-negative embryos, respectively (A' vs. B'; $\mathrm{C}^{\prime}$ vs. $\left.\mathrm{D}^{\prime}\right)$. Arrows in $\mathrm{C}^{\prime}$ and $\mathrm{D}^{\prime}$ indicate Otx2 expression that extends to the midbrain-hindbrain boundary (Kablar et al., 1996). 

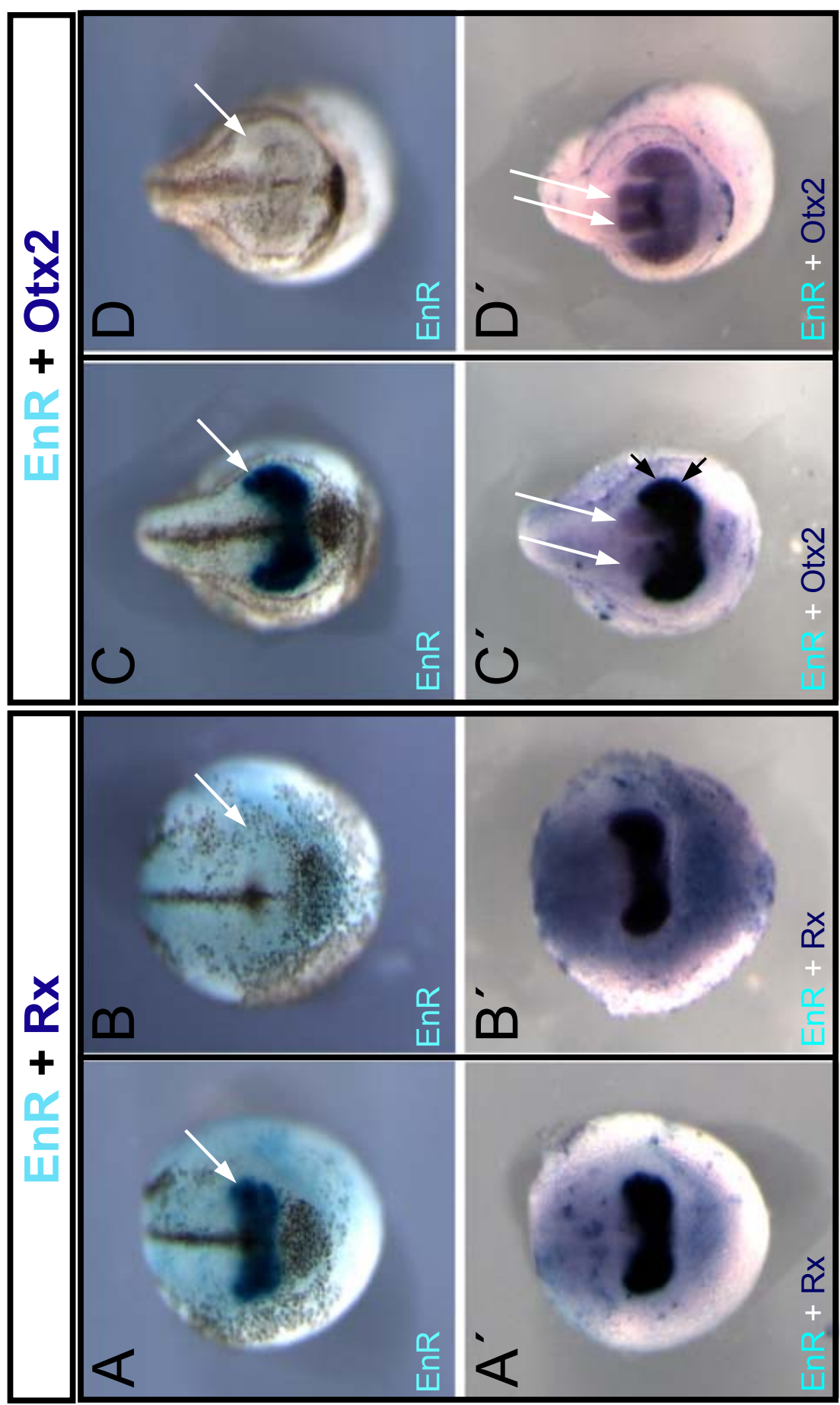
Figure 3-7. Comparison of native $O t x 2, R x$ promoter-driven $O t x 2$-EnR and native $R x$ expression patterns by double whole-mount in situ hybridization. Embryos shown here are from the cross described in Fig. 3-3. In A-D and H-K, the dark blue stain is the expression pattern of $\operatorname{Otx} 2$-EnR, while the light purple stain is the expression of $\operatorname{Otx} 2$, at indicated stages. Similarly, in E-F and L-M, the dark blue stain is the expression pattern of $R x$, while the light blue stain is the expression of Otx2. Dotted lines indicate the outline of Otx2 expression. (A, C, H, J) Otx2-EnR-negative embryos showing Otx2 expression only. An arrow in $\mathrm{C}$ points to the 'hole' in the middle of the Otx2 expression domain, where the Otx2 expression is greatly downregulated. An arrow in $\mathrm{J}$ indicates the Otx2 expression in the cement gland anlage. $(\mathrm{G}, \mathrm{N})$ A schematic diagram illustrating the expression patterns of $O t x 2$ (blue) and $R x$ (red) overlapping in the presumptive retinal field at stage $15(\mathrm{G})$ and $18(\mathrm{~N})$. 


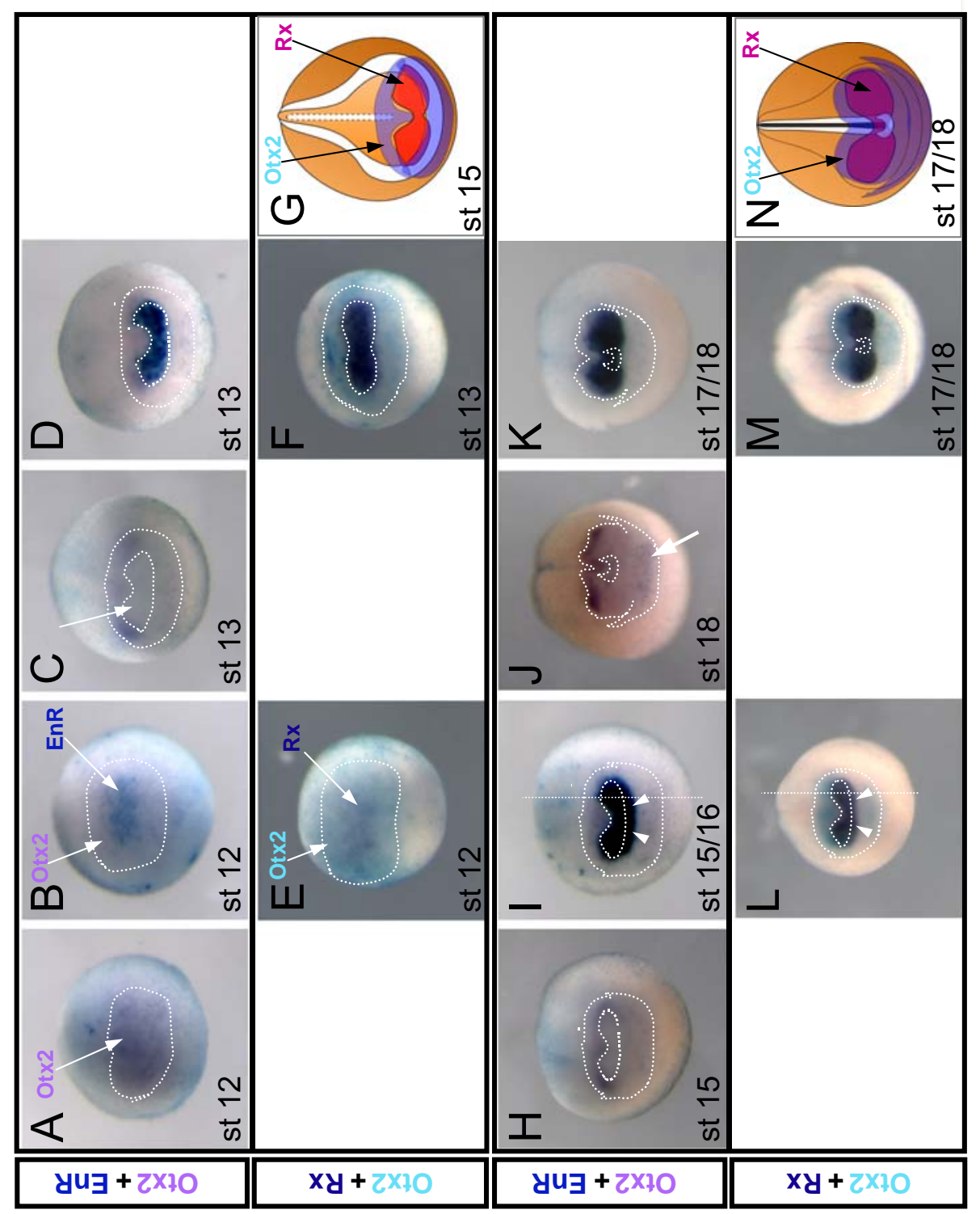


Figure 3-8. Expression of Pax6 and Otx2-EnR in RU486-treated embryos from the cross described in Fig. 3-3 at st 31/32 as in wholemount and in sections after whole-mount double in situ hybridization using Pax6 and EnR probes. Embryos were cohybridized with both probes and visualization of each probe was carried out sequentially. Otx2-EnR expression was first revealed by light blue staining $(A, D)$ followed by the detection of Pax6 RNA by dark staining (A', D'). In all panels, dorsal is at the top. (A, D) Lateral views of stage 31/32 Otx2-EnR-expressing (A) or control sibling (i.e. Otx2-EnRnegative; D) embryos. (A', D') Same embryos in A and D, respectively, showing Pax6 expression. The Pax6 staining in the developing eye (arrow) and overlying forebrain (open arrowhead) is evident. The region between arrowheads indicates the gap in Pax6 expression in the midbrain. Note that superficially, Pax6 expression in the eye and the brain does not seem changed by the induction of Otx2-EnR (compare $\mathrm{A}^{\prime}$ and $\left.\mathrm{D}^{\prime}\right) .(\mathrm{B}, \mathrm{C}$, E, F) Transverse sections of embryos in $\mathrm{A}(\mathrm{B}, \mathrm{C})$ and $\mathrm{D}(\mathrm{E}, \mathrm{F}) . \mathrm{C}$ and $\mathrm{F}$ are at more posterior plane of sectioning than $\mathrm{B}$ and $\mathrm{E}$, respectively. (E, F) The intensity of Pax6 staining is greatly reduced in the outer nuclear layer $(\mathrm{O})$ but maintained in the distal part of the retina. Black arrowheads indicate the elongated monolayer of RPE, which has begun to contact the invaginating dorsal neural retina (red arrowhead). (B, C) Pax6 staining appears more diffusely throughout the defective retina. The optic vesicle is connected abnormally to the brain through the lumen (open arrows). Open arrowheads in $\mathrm{C}$ and $\mathrm{F}$ point out the lens outlined by the dotted white line. o, outer nuclear layer. 

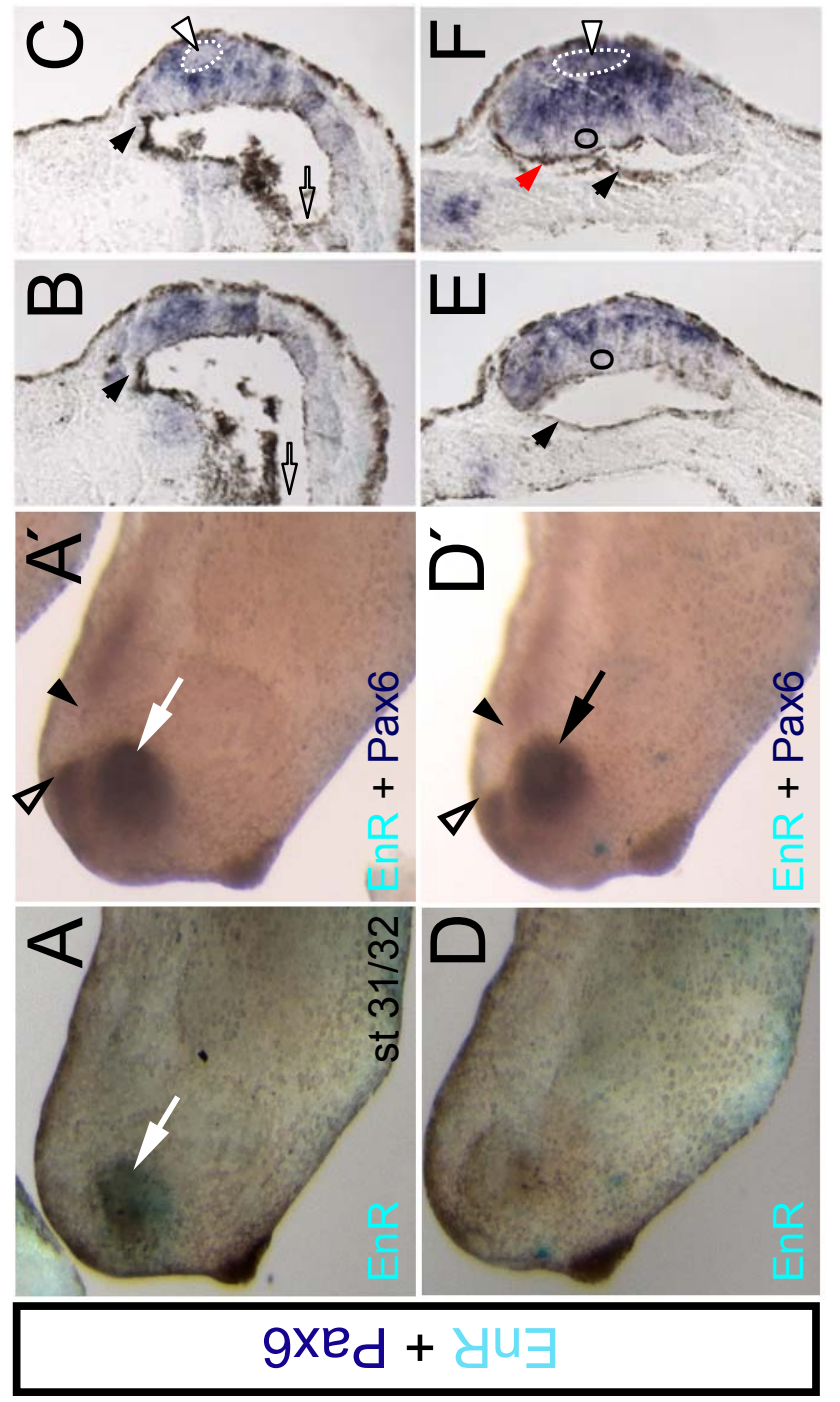
Figure 3-9. Localization of Pax6 in the optic cup of st $37 / 38$ embryos from the cross described in Fig. 3-3 in the presence of RU486. In all panels except (A), (F), and (K), dorsal is at the top; in (A, F, K), dorsal is to the right. Pax6 and EnR transcripts are detected in control UAS + (RFP+); GALPR- (A-D) and UAS+; GALPR+ (F-P) embryos by double in situ hybridization for EnR and Pax6, as described in Fig. 3-8. (A, F, K) Lateral views of st 37/38 EnR-negative control (A) and EnR-positive mildly affected (F) and severely affected (K) embryos. Arrows point out the Pax6 staining in the forebrain. (B, G, L) Enlarged views of insets in E, I, and O, respectively, which are transverse sections of $\mathrm{A}, \mathrm{F}$, and $\mathrm{K}$, respectively. The arrowheads point out the staining in the lens epithelium. Note that staining is relatively uniform throughout the retina of a severely affected embryo while confined to the ganglion cell $(\mathrm{G})$ and the inner nuclear (I) layers in control retina. (C-E, H-J, M-P) Series of transverse sections, which are not consecutive, in an anterior to posterior order, of embryos in $A(C-E), F(H-J)$, and $\mathrm{K}(\mathrm{M}-\mathrm{P})$, respectively, at the level of forebrain (C-D, H-I, M-O) or midbrain (E, J, P). (C) Arrows indicate Pax6 expression throughout the dorso-ventral extent of the telencephalon. Note that a similar patterning (black arrow for dorsal; red for ventral) is also visible in the forebrain of mildly affected embryos at the similar level. (D, E) Optic cup encircled by the RPE is completely separated from the brain. Arrows in E indicates the ventral RPE. Note that at anterior levels, a continuous lumen (open arrows in H, I, M, O) abnormally connects the brain and the retina in Otx2-EnR-positive embryos. A black arrow in I indicates EnR expression that becomes patchy at this stage. The absence of the ventral RPE (black arrows in J, P) is apparent. G, ganglion cell layer; I, inner nuclear cell layer; Le, lens; NT, neural tube; O, outer nuclear cell layer, RPE, retinal pigmented epithelium. 


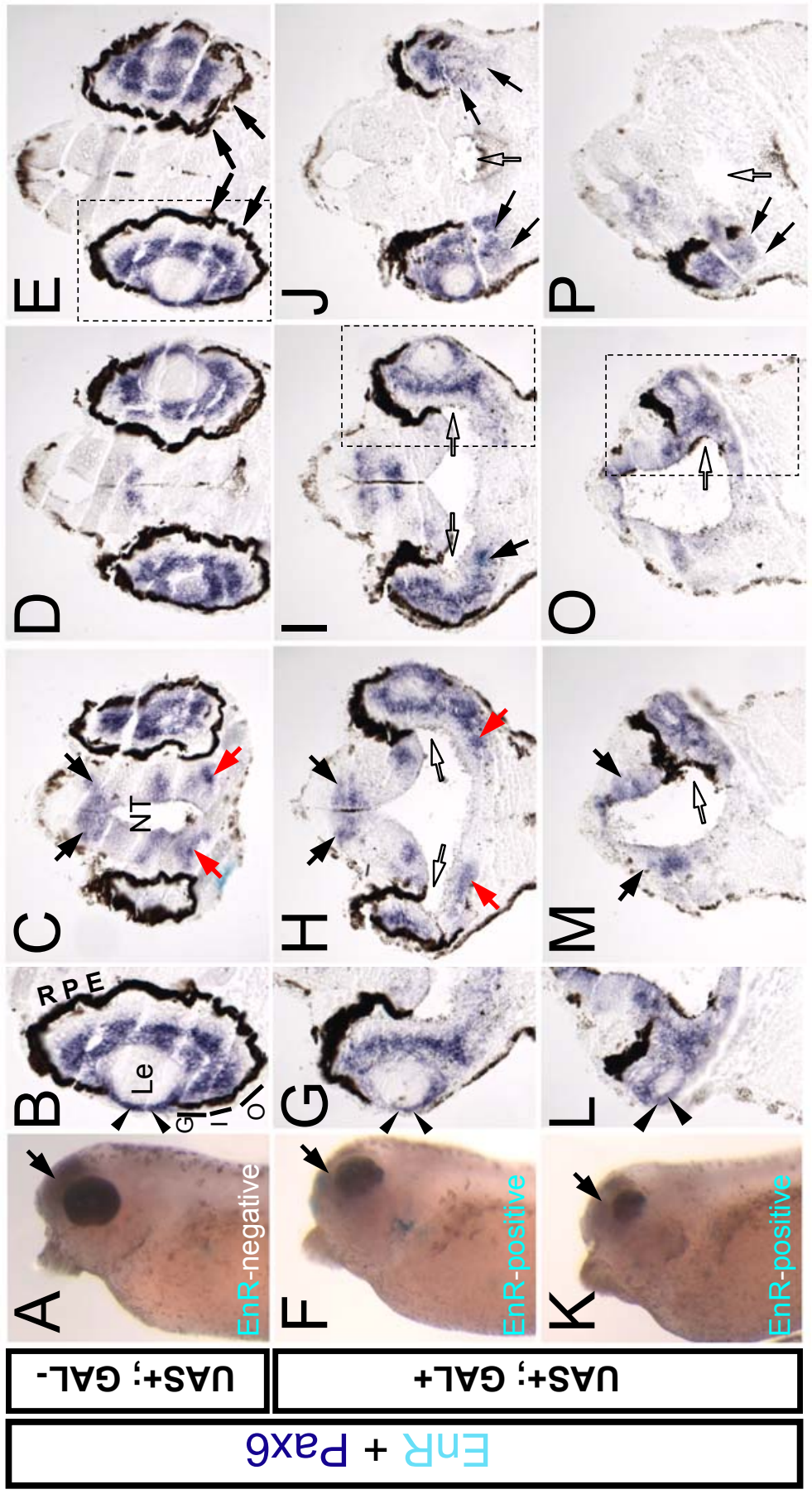


Figure 3-10. PAX6 and opsin localization in the optic cup expressing Otx2-EnR in the presence of RU486. Double immunostaining of transverse sections through the eye of control UAS+; GAL- (A-D) and UAS+; GAL+ (E-P) st 41 embryos using anti-Pax6 and anti-opsin antibodies. Two color immunofluorescence images were obtained for PAX6 (green; B, F, J, N) and opsin (red; C, G, K, O), as indicated. Each of two corresponding images was merged in (D, H, L, P). (A, E, I, M) Bright field images. The dotted white line $(\mathrm{N}, \mathrm{O})$ delienates the neural retina from the pigmented epithelium. Arrowheads in the red images indicate the ectopic opsin expression in cells outside of the photoreceptor cell layer. Note that colocalization of PAX6 and opsin is represented by orange color in cells (arrowheads) in the merged images. For genotyping, PCR analysis using individual embryos was performed with primers for RFP or GALPR. The red fluorescence in the lens is nonspecific (arrow in C). GC, ganglion cell layer; INL, inner nuclear layer; Le, lens; PR, photoreceptor cell layer. 


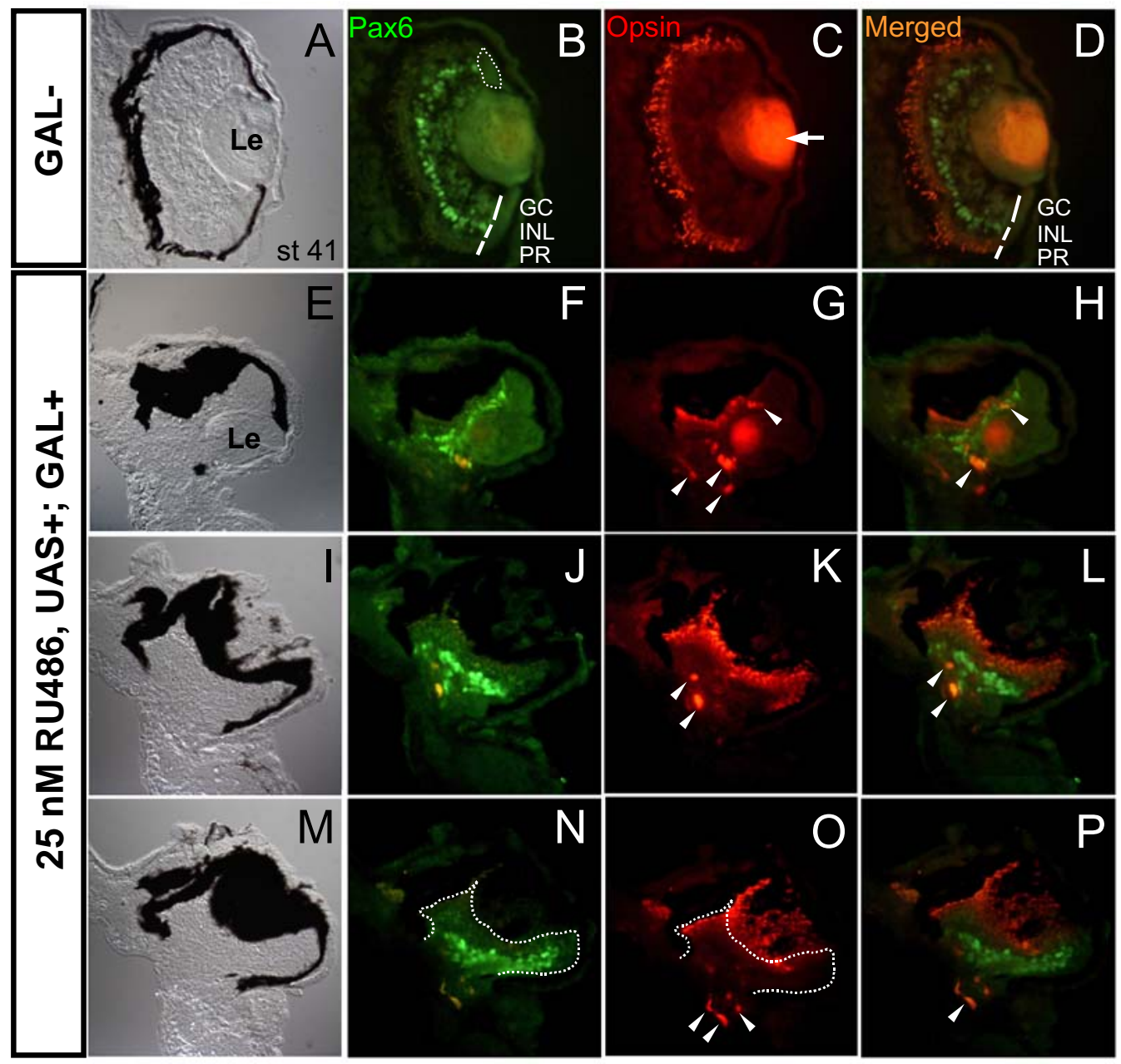


Figure 3-11. Lens development abnormalities in embryos expressing Otx2-EnR in the retina. (A-C) Cross sections through st 41 lens of RFP+ (UAS+); GALPR- (A) and RFP+ (UAS+); GALPR+ (B, C) embryos treated with $25 \mathrm{nM}$ RU486. Sections were stained with Hematoxylin to visualize nuclei. Dotted lines indicate the outline of the lens. (A) Open arrows indicate darkly stained lens epithelium. Arrowheads point to elongated, differentiated lens fibers in control lens. (B, C) Disorganized lens in mildly affected embryos. While the lens epithelium is present (open arrows), failure to form fully differentiated lens fibers is evident by the presence of clear nuclear staining in cells (arrowheads). Note that the wild-type lens fibers are normally denucleated as they differentiate. 

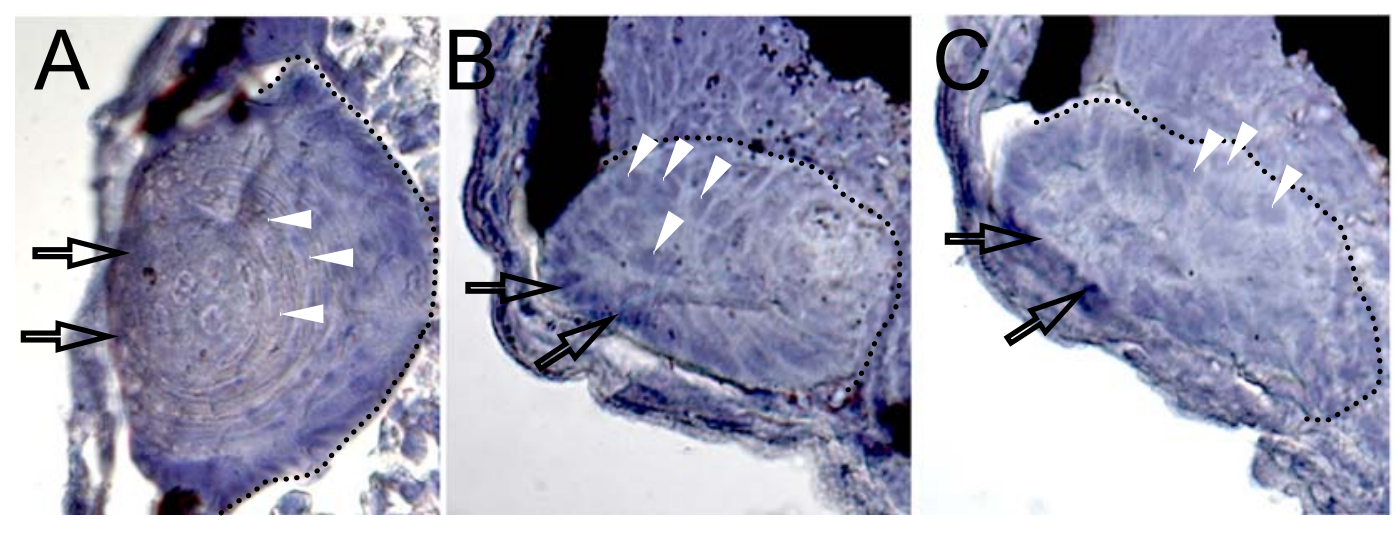


\section{Chapter 4:}

Discussion and future applications 
In this dissertation, I evaluated the utility and efficacy of a hormone-inducible binary gene targeting system in an emerging new vertebrate genetic model, Xenopus tropicalis. I have shown that the GAL4 activator and UAS target lines used in this study were able to drive a rapid RU486-dependent expression of GFP reporter and dominant inhibitory effector genes in a tissue-specific manner. Moreover, the expression of dominant negative Otx2-EnR was induced to levels capable of efficiently repressing the OTX2 activity, resulting in aberrant eye morphogenesis. Therefore, I conclude that this hormone-inducible regulatory system works efficiently in $X$. tropicalis and this system should be readily applicable for other gene function studies at all desired stages of development.

\section{Otx2 gene is required for multiple steps of eye morphogenesis}

Evidence from several different sources, especially from recent studies using Otx mutant mice, indicates that $O t x 2$ is involved in multiple steps of vertebrate eye formation, including the determination of the early eye field formation, the morphogenesis of the eye cup, and the differentiation of specific retinal neurons (Bovolenta et al., 1997; MartinezMorales et al., 2001; Nishida et al., 2003; Simeone et al., 1993; Viczian et al., 2003). Results from this study also support the idea that Otx2 plays an important role throughout the different steps of eye formation and particularly in eye morphogenesis.

In Otx2-EnR-expressing embryos, the elongation of the optic vesicle is severely impaired. As a result, first, the future RPE cells remain dorsally in a thick multi-cellular layer in contrast to the wild-type retina, where RPE cells spread to surround the neural retina in a single layer of cells. Second, expression of the dominant negative construct 
leads to the failure of the optic cup detaching from the neural tube because this dorsal movement normally leaves the eye attached to the neural tube only through a thin optic stalk at the ventral pole. Later, cell movements responsible for the completion of mature optic cup, i.e. formation of the ventral retina, also appear to be defective as a partial or complete absence of the ventral RPE is observed in these induced mutant embryos. In addition, optic fissure morphogenesis is also impaired. In conclusion, different steps of retinal morphogenesis seem to be disrupted in embryos expressing the Otx2-EnR in the developing retina, suggesting Otx2's involvement in eye morphogenesis.

In agreement with these results, the putative role of Otx2 in cell morphogenesis has been implied in other tissues during development. For example, before gastrulation, mouse Otx2 is required for anteriorly directed morphogenetic movement of the anterior visceral endoderm (AVE), which in turn, influences the adjacent ectoderm to form anterior brain as a 'head organizer' (Acampora et al., 1995; Perea-Gomez et al., 2001). Another example is its involvement in controlling oriented embryonic cell movements during Xenopus gastrulation. Xenopus Otx2 excludes the early brain cells expressing this gene from undergoing convergent extension movements (Morgan et al., 1999), which involve cell-shape changes and directed cell movements (Keller, 1991; Keller, 2002). As a result, the brain remains relatively broad and short while the remaining body axis including the spinal cord forms a narrow, elongated array after gastrulation. Studies in Xenopus revealed that a Xenopus homolog of the calponin gene, XclpH3, is a direct target of Otx 2 and mediates the exclusion from convergent extension movements by Otx2. As its expression pattern implies, $\mathrm{XclpH} 3$ might be involved in another morphogenetic process, head mesoderm migration, during gastrulation (see below). This gene product 
binds actin and myosin, and prevents the sliding of actin filament on myosin (Morgan et al., 1999).

Despite a possible critical role of Otx2 for controlling morphogenesis of different tissues, its underlying molecular mechanisms remain to be established. Since Otx2 has a number of different roles in early development, it is believed to control multiple downstream target genes, directly or indirectly (reviewed by Boncinelli and Morgan, 2001). In support of its possible function in regulating morphogenetic cell movements and cell shape changes involved in different developmental processes, some of these Otx2 target genes identified from different sources encode proteins implicated in cell-cell interaction and extracellular adhesion, such as cadherins and calponins described above (Boncinelli and Morgan, 2001 and references therein). For example, a reduction of expression of $R$-cadherin and ephrin-A2, implicated in regulating adhesive interactions of neuroepithelial cells, is observed in mutant cells of Otx2-/-/wild-type chimeric mice (Rhinn et al., 1999). In addition, a recent microarray analysis using Drosophila overexpressing either otd or human Otx2 (Montalta-He et al., 2002), and a serial analysis of gene expression (SAGE) assay comparing gene expression profiles from wild-type and Otx2-/- gastrulating mouse embryos (Zakin et al., 2000), also have revealed genes implicated in cell adhesion and cell movement, such as catenin and tropomyosin, as putative Otx2 targets. Although it is not clear whether these genes are involved in morphogenesis of eye, functioning downstream of Otx 2 , these candidate molecules can be analyzed by further experiments (see below).

Different cadherins, homophilic cell adhesion molecules involved in cell sorting and tissue morphology, are dynamically expressed in different regions of the developing 
eye including lens and retina during eye development (Hatta and Takeichi, 1986; Takeichi, 1988; Xu et al., 2002). Thus, it would be possible that some of the Otx2 targets with similar functions control cell adhesive properties of retinal cells so that different groups of cells retain different tissue coherence and migrate in a concerted fashion during eye morphogenesis. In addition, the ectopic expression of Otxl and otd results in strong cell aggregation of descendants of the injected cell in zebrafish (Bellipanni et al., 2000).

As the optic cup develops, there are dramatic cell shape changes. Relevant to the studies here, the dorso-proximal portion of the optic vesicle, representing the prospective pigment layer of retina is initially a few cells-thick and consists of columnar cells (Li et al., 2000; Svoboda and O'Shea, 1987). Later, as the vesicle elongates dorsally (see black arrows in Fig. 4-1A and B) and invaginates (open arrow in Fig. 4-1B), cells flatten to become cuboidal or squamous, constituting a thin monolayer of the pigmented epithelium surrounding the neural retina (Hollyfield, 1973; Li et al., 2000; Svoboda and O'Shea, 1987). To form this narrower, flattened single layer of cells of the RPE, these cells might not only change shape but might also intercalate between one another. The considerably thicker dorsal RPE (black-colored regions in Fig. 4-1G and H), which consists of a disorganized multi-cellular layer, observed in the optic cup of Otx2-EnR-expressiong embryos indicates the disruption of these processes. A relatively simple cell biological experiment would reveal the nature of these cell movements and possible cell intercalations during eye morphogenesis: the lumen of early neural tube can be surfacelabeled with a fluorescent dye (Shook et al., 2004) and this staining in the monolayer of the presumptive RPE can be visualized later in development. If radial cell intercalation indeed occurs, stained cells will be found interspersed regularly between unstained cells. 
Since XclpH3, a calponin homolog, regulates a switch between the two morphogenetic processes, convergent extension and head mesoderm migration as a direct target of Otx2 (Morgan et al., 1999), it is possible that cell shape changes and cell intercalation during optic cup formation might be regulated by a similar molecular mechanism requiring the activity of $\mathrm{XclpH3}$, a actin/myosin-binding protein (Gusev, 2001). Spec $2 a$ is a sea urchin homolog of calponin, which is also a direct target of SpOtx, the sea urchin homolog of the vertebrate Otx2 (Hardin et al., 1985; Li et al., 1999). Activation of Spec2 also appears to be involved in controlling morphogenetic cell movements. However, $\mathrm{XclpH} 3$ is expressed both in the Xenopus optic vesicle undergoing morphogenesis and during head mesoderm migration (Morgan et al., 1999). Thus, it is unknown at present whether $\mathrm{XclpH3}$ reorganizes cytoskeleton molecules including microfilaments in a different way to promote cell movements or if $\mathrm{XclpH} 3$ expression in the eye and the forebrain prevents these tissues from undergoing the convergent extension movements while another molecule(s) is involved in the retinal cell movement for the formation of the optic cup. Future studies addressing the molecular nature of cell movements during eye formation remain to be performed to elucidate the precise role of $\mathrm{XclpH} 3$ or molecules with a similar function in regulating these morphogenetic processes.

As discussed above, a number of genes implicated in cell-cell adhesion and cell movements have been identified as putative targets of Otx2 on the basis of their up- or down-regulation in microarray and SAGE analyses, and Otx2 mutant mice. Therefore, in order to identify Otx2 targets during eye morphogenesis, first, alterations in the expression of these genes including $\mathrm{XclpH} 3$ in the induced mutant embryos expressing Otx2-EnR can be tested by in situ hybridization analysis. Simultaneously, computational 
sequence analyses also can be performed to identify putative Otx2 binding sites in $5^{\prime}$ regulatory sequences of these genes, which are obtainable from the JGI Xenopus tropicalis genome project site (http://genome.jgi-psf.org/cgi-bin/runBlast?db=xenopus0). Once confirmed by either approach above, those putative Otx2 target genes can be subjected to further experimental analyses including transgenic experiments targeting expression of gene products in the eye, which are expected to phenocopy or rescue Otx2EnR-induced phenotypes. As I have generated effector lines carrying the wild-type Otx2 (UAS-Otx2) or a chimeric constitutively active form of Otx2 consisting of the Otx2 DNA binding domain fused to the VP16 activation domain (UAS-Otx2-VP16), it is possible to make double effector lines bearing UAS-Otx2-EnR and UAS-Otx2. Thus, it will be interesting to see whether crosses of UAS-Otx2-EnR; UAS-Otx2 double UAS lines with a pRx-GALPR activator line will rescue the phenotype caused by Otx2-EnR. In addition, UAS-Otx2-VP16 lines, when crossed to the activator lines, also will provide unique information on Otx2 function as the constitutive active Otx2-VP16 construct dominantly inhibits endogenous Otx2 function as a transcriptional repressor, enhancing or not affecting its function as an activator.

Changes in the expression of genes that contribute to the early compartmentalization of the optic vesicle could be, at least in part, responsible for the morphological consequences of Otx2-EnR-expressing embryos (Martinez-Morales et al., 2001). One of the striking phenotypes caused by the induced expression of Otx2-EnR is a failure of the detachment of the optic cup from the neural tube. The overexpression of Xenopus vax2, which is normally expressed in the ventral part of developing retina including the optic stalk territory, has been shown to have a ventralizing effect on the 
developing eye (Barbieri et al., 2002; Barbieri et al., 1999). In resultant embryos, the optic cup and the forebrain were abnormally connected by an enlarged optic stalk, which is distinct from, yet similar to the phenotype observed in Otx2-EnR-expressing embryos with no recognizable optic stalk structure. Therefore, it will be helpful to examine the expression of vax2 in the Otx2-EnR-induced mutant embryos. In addition, the dorsal expansion of the expression of another molecular marker of the ventral retina, $\operatorname{Pax} 2$ was observed in otx mutant mice (Martinez-Morales et al., 2001). This could be explained by the expansion of Pax2-positive territory in the dorsal retina in the absence or reduction of the mouse Otx2 whose expression is normally restricted to the dorsal half of the optic vesicle, unlike uniformly-expressed Xenopus Otx2 (Kablar et al., 1996; Zygar et al., 1998). Nonetheless, possible alterations in the expression of genes involved in the establishment of a dorsoventral pattern of the early eye vesicle, such as Pax2 (Heller and Brandli, 1997; Nornes et al., 1990; Torres et al., 1996) and Mitf (Kumasaka et al., 2004; Mochii et al., 1998) can be examined to test the hypothesis that disruption of the early patterning of eye territories is responsible for the phenotype caused by the Otx2-EnR expression. This seems unlikely, however, since the early partitioning of the developing retina in these embryos seems normal with the RPE and neural retina developing in the correct regions fated to become these structures despite the morphological abnormalities.

Although quite variable, the phenotypes caused by Otx2 repression generally included abnormalities in the development of the optic fissure. Closure of the choroid fissure is an important event in vertebrate eye development. Only after the fissure is closed, does the formation of vitreous begin. Growth and osmotic activity of the vitreous body creates the intraocular pressure (IOP), which plays a significant role in eye 
formation (Beebe, 1986). IOP is required for the normal development of the RPE and cornea, and the overall growth of the eye. Therefore, the later phenotypes including abnormal retinal layering and the absence of RPE observed in the optic cup of the Otx2EnR-induced mutant embryos could be due to secondary effects of the failure of optic fissure closure, rather than due to the direct effects of low levels of OTX2. Therefore, further experiments would clarify later Otx2 function for optic cup formation, independent of the optic fissure closure: Otx2 activity can be inhibited without affecting the fissure development, by administering RU486 after st 35/36 when the fissure becomes closed in Xenopus (Nieuwkoop and Faber, 1967).

As Otx2 activity is reduced in both retina and lens of Otx2 mutant mice (Martinez-Morales et al., 2001; Matsuo et al., 1995), it was not clear whether the observed defects in lens development were due to lens cell-autonomous effects of Otx2 mutation. However, similar abnormalities in lens differentiation caused by the retinaspecific repression of Otx2 using the inducible regulatory system indicate the importance of the retina as a potential source of lens-inducing signal(s). At present, the precise molecular mechanism that underlies this indirect function of Otx2 is unknown. One simple explanation would be that Otx2 is involved in activating, either directly or indirectly through another transcription factor, the expression of downstream molecule(s) in the optic vesicle, which function as a lens-inducing signal. Candidates for lensinducing molecules include members of the fibroblast growth factor (FGF) that play an important role in the induction of lens cell proliferation and fiber differentiation (Chow and Lang, 2001; Lovicu et al., 1997; Lovicu and McAvoy, 2001; McAvoy et al., 1999). As the neural retina is believed to be a potential source of FGF, abnormal neural retina of 
Otx2-EnR-exprssing embryos would explain failure of lens fiber cell differentiation, the main lens defects observed in these embryos. Another possibility is that the lens malformation observed in the Otx2-EnR-expressing embryos could result from defective ciliary body formation. There are suggested reciprocal interactions between lens and the ciliary body at the rim of the optic cup (Beebe, 1986). Since a disorganized retina was observed, ciliary body development also could be disturbed by the induced repression of Otx2, leading to abnormal lens development. Although this explanation may not be the primary cause of lens defects, because some of the mildly affected embryos with a relatively normal retina also showed the reduced, disorganized lens, the presence of the ciliary body remains to be tested using CMZ-specific markers, such as $R x$ and XNotch- 1 (Perron et al., 1998).

\section{$O t x 2$ and retinal differentiation}

In addition to morphogenetic abnormalities, Otx2-EnR expressing embryos displayed defects in retinal cell layering and differentiation including alterations in expression of opsin and Pax6. The acquisition of a specific cell fate and morphogenesis are two mutually coordinated, interdependent developmental processes required for the formation of various tissues and organs. Vertebrate eye formation, particularly, the folding of the optic vesicle to the optic cup involves complicated morphogenetic cell movements and changes in cell shape. The basic structure of double-layered eye cup resulting from these morphogenetic movements would be a prerequisite for the proper maintenance and differentiation of cell identity which are, in turn, achieved by the coordinated expression of transcription factors and secreted inductive molecules from 
cells in the properly positioned tissues. While it is known that all the retinal cell types are generated from multipotent precursor cells (Holt et al., 1988), it has been proposed that vertebrate retinal cell fate determination occurs in an orderly sequence by intrinsic regulators and environmental signals (reviewed by Livesey and Cepko, 2001). For example, correct differentiation and lamination of the neural retina require the presence of adjacent RPE, in addition to intrinsic competence (Bovolenta et al., 1996; Frade et al., 1996; Raymond and Jackson, 1995): an ablation of the RPE in transgenic mice resulted in disruption of the retinal layers (Raymond and Jackson, 1995). Interestingly, the laminar structure of the retina was maintained only adjacent to patches of escaped RPE cells in these mice. In addition, diffusible molecules, such as neurotrophin-3 and insulin-like growth factor-1, which promote neural retina differentiation in vitro, are synthesized in the RPE and diffused into neuroepithelium (Bovolenta et al., 1996; Frade et al., 1996). Thus, the complete or partial absence of the ventral RPE in Otx2-EnR-expressing embryos might account for disorganized retinal layering and the loss of opsin expression in the ventral retina. As mentioned above, Otx2 conditional knockout mice in which the Otx2 gene is deleted under the control of the photoreceptor cell-specific Crx promoter showed a loss of the photoreceptor cells. In contrast, the dorsal side of the retina expressing Otx2-EnR, which has the thickened yet, morphologically recognizable RPE, showed relatively normal opsin expression, suggesting the presence of photoreceptors. This phenotypic difference could be explained by the differences in the expression pattern of Otx2 cognates between the two species, as Xenopus Otx2 is only expressed in bipolar cells while the mouse cognate is in both photoreceptor and bipolar cells. Alternatively, patchy Otx2-EnR expression observed only in the small number of retinal 
cells especially at later optic cup stages might not be sufficient to interfere broadly with later Otx2 function involved in photoreceptor cell differentiation.

While Otx1-/-; Otx2-/+ mice failed to form RPE (Martinez-Morales et al., 2001), differentiation of dorsal RPE did not seem severely affected in embryos expressing Otx2EnR. This difference also could be explained by the expression patterns of Xenopus Otx2 and $R x$ whose promoter drives the expression of $O t x 2$-EnR. Both Otx2 and $R x$ are expressed in the entire retinal neuroepithelium of the early optic vesicle (st 19-22). Later, while Otx2 continues to be expressed throughout the presumptive RPE and neural retina during subsequent stages until later optic cup stages, $R x$ expression becomes confined to the neural retina and not in the RPE at similar stages. Thus, Otx2-EnR expression appears to be nonoverlapping with Otx2 in the developing RPE except early optic vesicle stages, allowing the later differentiation of the pigmented epithelium.

In addition to the loss of opsin expression in the outer layer of the ventral retina, there was ectopic opsin expression observed in the inner retinal layers of Otx2-EnR expressing embryos. One possible explanation could be that Otx2 normally suppresses the opsin expression in the bipolar layer, and this repression is relieved by Otx2-EnRmediated inactivation of the Otx2. Repression of the $O t x 5 b$-driven expression of an opsin reporter by Otx2 supports this hypothesis (Viczian et al., 2003). In Otx2-EnR expressing induced mutant embryos, despite the morphological abnormalities, Pax6 expression does not seem to be severely altered although most severely affected embryos showed a diffuse expression throughout the retina instead of being confined to inner and ganglion cell layers as in wild-type controls. It is not clear whether Pax6 expression is independent 
of Otx2 expression or patchy Otx2-EnR expression is not sufficient to influence Pax6 activation in a globally discernable way.

\section{Model for eye morphogenesis and Otx2}

A schematic illustration comparing eye morphogenesis in normal and Otx2-EnRexpressing embryos is represented in Fig. 4-1. In this simplified diagram, the folding of optic vesicle into the optic cup and cell movements involved in this progressive process are drawn in transverse sections. This proposed model takes into account all the data previously reported (Holt, 1980) and described in this study. During normal Xenopus development, eye rudiment first becomes visible as an outpocketing of the ventral diencephalon, which enlarges to form the optic vesicle. Subsequently, morphogenesis of the optic cup is initiated after the optic vesicle contacts the overlying presumptive lens ectoderm. The dorso-proximal part of the vesicle (dark gray-colored region in Fig. 4-1A), then elongates dorsally (black arrows in Fig. 4-1A and B). As a consequence of this dorsal movement, the eye vesicle now remains attached to the brain only by a narrow optic stalk at the ventral pole (Fig. 4-1B). Then, the distal part of the optic vesicle (yellow-colored region in Fig. 4-1B), the prospective neural retina, invaginates (open arrow in Fig. 4-1B), bringing itself toward the proximal wall of the vesicle (dark graycolored region in Fig. 4-1B), the future RPE. The presumptive RPE at this stage has become a thin monolayer of flat cells, presumably as a result of cell intercalation. Along with this retinal invagination, the proximal and distal, i.e. the outer and inner, layers of the optic cup come into close contact with each other, thereby obliterating the primary eye cavity between the layers. 
The optic fissure forms from the distal margin of the vesicle (arrowhead in Fig. 41B) during st 29-31 and closes at st 35/36. During intervening period, retinal cells move ventrally (arrows in Fig. 4-1C) to form the complete of the optic cup, a set of movements especially important for the formation of the ventral retina. By this downward movement, the lower half of the eye develops and gradually grows to the size of the dorsal half (Fig. 4-1D). In this way, the double-walled eye cup finally forms, whose considerably thicker distal wall differentiates into various retinal neurons arranged in three distinct layers, whereas the proximal layer forms the RPE encircling the entire neural retina, except the opening to the exterior, the future iris. As the optic vesicle is transforming into an optic cup, the ectoderm immediately overlying the cup undergoes an associated change: a thickening to form the lens placode (Fig. 4-1B and C) that later further develops into the differentiated lens (Fig. 4-1D) consisting of transparent lens fibers and a permanent lens epithelium of the outer wall (not shown in this model). Numerous downstream targets of Otx2 including molecules implicated in cell-cell or cell-extracellular adhesion could be involved in this dynamic process of eye morphogenesis.

In contrast, in embryos expressing RU486-induced Otx2-EnR in the developing retina, the different steps of eye morphogenesis described above are severely affected. Molecular pathways requiring the activity of $O t x 2$ target genes controlling cellular coherence and migration might be responsible for the abnormal morphogenesis of the optic cup expressing Otx2-EnR as discussed earlier. The initial lateral protrusion and subsequent dorsal elongation of the optic vesicle, presumably driven by cell shape change and cell intercalation, appear defective in these embryos (E, F) although the earliest regionalization of each compartment in the vesicle seems to occur (i.e. color-coded for 
different retinal fates). The eye vesicle remains abnormally connected with the brain, forming a broad central lumen (F). The optic fissure is never closed, and a considerably thickened pigmented epithelium remains dorsally localized (G). During the later phase of optic cup formation, the downward expansion of the ventral half of the retina seems affected, resulting in a ventral retina that is significantly reduced in size (compare D and H). Absence or morphological abnormalities of the lens tissue is also observed. However, this abnormally folded retina seems to undergo neural differentiation to some extent, as it shows an opsin-positive photoreceptor layer dorsally. However, loss of the ventral photoreceptors in the absence of the ventral RPE suggests that the basic morphology of double-layered eye cup achieved through dynamic morphogenetic cell movements might be required for final differentiation into proper retinal cell types, which is coordinated by the cell autonomous expression of transcription factors and secreted inductive molecules from neighboring cells in the properly positioned tissues.

Future investigations will be needed to elucidate the molecular pathways responsible for the morphogenetic defects in Otx2-EnR-expresing embryos and to establish the precise roles of Otx2 and its downstream targets during eye morphogenesis.

\section{Future Applications}

The RU486-dependent regulatory system described in this dissertation represents a very powerful tool for addressing a variety of important biological questions. This method, combined with a dominant-negative strategy, can be used to delete specific gene activities in defined subsets of cells and at a desired developmental stage. It can also be used for gain-of-function studies by ectopically expressing gene products as over- or 
misexpression phenotypes are extremely informative about gene function as well (Rorth, 1996; Rorth et al., 1998). Therefore, this system offers a variety of applications to study how activation and ablation of specific gene products at different developmental time points might affect cell differentiation and the development of an organism. For example, a transcriptional activator can be expressed in a different subset of cells or in endogenous target cells but at a different time, and then its effect on subsequent cell differentiation or development of an organ can be assayed without affecting other tissues, unlike ubiquitous RNA injection assays. In this way, one can determine if the gene activity is necessary and sufficient for the specification of cell fate and tissue determination.

Moreover, this system has advantages for loss-of-function studies over conventional gene targeting technique in mice. Problems of knockouts of multifunctioning genes important for the development of central nervous system (CNS) include lethality in early embryonic stages. This problem can only be overcome by conditional inactivation or deletion of target gene in a tissue- and stage-specific fashion. In addition, temporal control of this inducible system permitting activation of target gene expression at any desired developmental time point has an advantage over the Cre/loxp recombination method which causes irreversible permanent activation dependent upon a tissue-specific promoter used. It also should be mentioned that application of this system in Xenopus is particularly beneficiary since after gene inactivaiton or overexpression experiments, frog embryos allow further embryological assays, some of which are extremely challenging in small inaccessible mouse embryos. As RU486 penetrates the epidermal barrier efficiently, and thus can be widely distributed throughout an organism (Burcin et al., 1998; Chae et al., 2002; Osterwalder et al., 2001; Roman et al., 2001), the 
RU486-inducible regulatory system should be effective for elucidating functions of genes involved in a variety of tissues and organs including the CNS.

Clinical gene therapy is another important potential future application of this system. For example, this system can be used for the generation of transgenic animals to establish a clinical model for cancer induction by conditional expression of oncogenes known to be involved in tumor development (Burcin et al., 1998; Kwak et al., 2004). Using tissue-specific promoters, the hormone-inducible activator can restrict the expression of an oncogene, a target for the regulator, to common cancer-developing tissues like the lung, skin or intestines. Since in this model, tumor formation can be induced in a specific tissue at a specific time, this system would be very useful for the investigation of the initiation and progression of cancer. It also would be possible to develop potential gene therapeutic approaches to tumor development by targeted expression of tumor suppresser genes although there are general concerns about transgenic approach including potential insertional mutations and unintended effects of transgene expression. Similar approaches should be applicable for investigating the developmental function of genes potentially involved in the pathogenesis of neurodegenerative diseases such as Alzheimer's disease (Feany, 2000; Link, 2001).

As there are general concerns about the specificity of dominant negative constructs (discussed in Chapter 3), another option in the conditional inactivation of gene activity is gene silencing using small RNAs, so called RNA interference (RNAi). In RNAi, exogenously added double-stranded RNA (dsRNA) and its derivatives, small interfering RNA (siRNA) direct the destruction of homologous cellular mRNA with very high sequence-specificity (Montgomery, 2004; Tijsterman and Plasterk, 2004). At 
present, RNAi-mediated gene inactivation is arguably, the most widely used reverse genetics technique to analyze the functions of genes in diverse organisms. When combined with the hormone-inducible GAL4/UAS system (Enerly et al., 2003; Lee and Carthew, 2003), RNAi (GAL4/UAS-RNAi) will allow temporally and spatially controlled silencing of a specific target gene. The initial test of the utility of RNAi in $X$. tropicalis would involve the generation of a line carrying UAS-dsRNA or siRNA against GFP on the genetic background of pRx-GFP. This animal could be crossed with the activator line bearing pRx-GALPR to see whether GFP activation in the eye is blocked in a ligand-dependent manner. In the future, once generated, UAS-RNAi lines targeting a variety of different genes could be utilized in combination with various GAL4 driver lines to inactivate genes in different tissues. Generation of libraries of these GAL4 driver and UAS-RNAi lines will also be invaluable resources to the Xenopus community.

Another method for the conditional deletion of genes would be the incorporation of the well-characterized Cre-loxP recombination system with the RU486-modulated system. Chimeric Cre recombinases with the ligand-binding domain of several nuclear steroid hormone receptors including the progesterone-binding domain have been generated and tested in transgenic mice (reviewed in Albanese et al., 2002; Jaisser, 2000). As the applicability of the tissue-specific Cre transgene-mediated recombination has been tested in the Xenopus system (Gargioli and Slack, 2004; Ryffel et al., 2003), the use of RU486-inducible Cre recombinase will add another level of temporal control of the transgene expression. For example, one could generate one transgenic line carrying a tissue-specific promoter driving RU486-inducble chimeric Cre and a second line carrying a ubiquitous promoter linked to a stop codon flanked by Cre-recognizing loxP sites fused 
to a downstream target gene. In this way, the target gene transcript is present ubiquitously, but its translation is blocked by the interfering stop codon. Only when RU486 is present, activated Cre excises the stop codon and the target protein is made in a tissue-specific manner. As the Cre activation by a single administration of RU486 causes irreversible recombination and induction of the tissue-specific target activation by a strong ubiquitous promoter, it does not require continuous exposure to the ligand or prolonged activation of the Cre protein. Thus, this method would be useful for dominant negative or RNAi experiments requiring very high levels of overexpression. A similar hormone-inducible Cre system with GFP reporter as target would allow accurate cell fate mapping as RU486 could be added at a specific developmental time point and GFP expression in certain precursor cells and their descendents could be followed from that point onward (Gargioli and Slack, 2004).

These future applications of the hormone-inducible system allowing both temporal and spatial control of transgenes will offer many more elaborate gene manipulation tools to dissect the functions of a gene product at different developmental stages in specific tissues during development, a level of gene function analysis which was not previously possible in Xenopus.

\section{References}

Acampora, D., Mazan, S., Lallemand, Y., Avantaggiato, V., Maury, M., Simeone, A. and Brulet, P. (1995). Forebrain and midbrain regions are deleted in Otx2-/- mutants due 
to a defective anterior neuroectoderm specification during gastrulation. Development 121, $3279-90$

Albanese, C., Hulit, J., Sakamaki, T. and Pestell, R. G. (2002). Recent advances in inducible expression in transgenic mice. Semin Cell Dev Biol 13, 129-41.

Barbieri, A. M., Broccoli, V., Bovolenta, P., Alfano, G., Marchitiello, A., Mocchetti, C., Crippa, L., Bulfone, A., Marigo, V., Ballabio, A. et al. (2002). Vax2 inactivation in mouse determines alteration of the eye dorsal-ventral axis, misrouting of the optic fibres and eye coloboma. Development 129, 805-13.

Barbieri, A. M., Lupo, G., Bulfone, A., Andreazzoli, M., Mariani, M., Fougerousse, F., Consalez, G. G., Borsani, G., Beckmann, J. S., Barsacchi, G. et al. (1999). A homeobox gene, vax2, controls the patterning of the eye dorsoventral axis. Proceedings of the National Academy of Sciences of the United States of America 96, 10729-34. Beebe, D. C. (1986). Development of the ciliary body: a brief review. Trans Ophthalmol Soc $U K \mathbf{1 0 5}, 123-30$.

Bellipanni, G., Murakami, T., Doerre, O. G., Andermann, P. and Weinberg, E. S. (2000). Expression of Otx homeodomain proteins induces cell aggregation in developing zebrafish embryos. Developmental Biology 223, 339-53.

Boncinelli, E. and Morgan, R. (2001). Downstream of Otx2, or how to get a head. Trends Genet 17, 633-6.

Bovolenta, P., Frade, J. M., Marti, E., Rodriguez-Pena, M. A., Barde, Y. A. and Rodriguez-Tebar, A. (1996). Neurotrophin-3 antibodies disrupt the normal development of the chick retina. $J$ Neurosci 16, 4402-10. 
Bovolenta, P., Mallamaci, A., Briata, P., Corte, G. and Boncinelli, E. (1997).

Implication of OTX2 in pigment epithelium determination and neural retina differentiation. J Neurosci 17, 4243-52.

Burcin, M. M., BW, O. M. and Tsai, S. Y. (1998). A regulatory system for target gene expression. Front Biosci 3, c1-7.

Chae, J., Zimmerman, L. B. and Grainger, R. M. (2002). Inducible control of tissuespecific transgene expression in Xenopus tropicalis transgenic lines. Mech Dev 117, 23541.

Chow, R. L. and Lang, R. A. (2001). Early eye development in vertebrates. Annu Rev Cell Dev Biol 17, 255-96.

Enerly, E., Larsson, J. and Lambertsson, A. (2003). Silencing the Drosophila ribosomal protein L14 gene using targeted RNA interference causes distinct somatic anomalies. Gene 320, 41-8.

Feany, M. B. (2000). Studying human neurodegenerative diseases in flies and worms. $J$ Neuropathol Exp Neurol 59, 847-56.

Frade, J. M., Marti, E., Bovolenta, P., Rodriguez-Pena, M. A., Perez-Garcia, D., Rohrer, H., Edgar, D. and Rodriguez-Tebar, A. (1996). Insulin-like growth factor-I stimulates neurogenesis in chick retina by regulating expression of the alpha 6 integrin subunit. Development 122, 2497-506.

Gargioli, C. and Slack, J. M. (2004). Cell lineage tracing during Xenopus tail regeneration. Development 131, 2669-79. 
Gusev, N. B. (2001). Some properties of caldesmon and calponin and the participation of these proteins in regulation of smooth muscle contraction and cytoskeleton formation. Biochemistry (Mosc) 66, 1112-21.

Hardin, S. H., Carpenter, C. D., Hardin, P. E., Bruskin, A. M. and Klein, W. H. (1985). Structure of the Spec1 gene encoding a major calcium-binding protein in the embryonic ectoderm of the sea urchin, Strongylocentrotus purpuratus. J Mol Biol 186, 243-55.

Hatta, K. and Takeichi, M. (1986). Expression of N-cadherin adhesion molecules associated with early morphogenetic events in chick development. Nature 320, 447-9. Heller, N. and Brandli, A. W. (1997). Xenopus Pax-2 displays multiple splice forms during embryogenesis and pronephric kidney development. Mech Dev 69, 83-104.

Hollyfield, J. G. (1973). Elimination of egg pigment from developing ocular tissues in the frog Rana pipiens. Developmental Biology 30, 115-28.

Holt, C. E. (1980). Cell movements in Xenopus eye development. Nature 287, 850-852. Holt, C. E., Bertsch, T. W., Ellis, H. M. and Harris, W. A. (1988). Cellular Determination in the Xenopus Retina is Independent of Lineage and Birth Date. Neuron 1, 15-26.

Jaisser, F. (2000). Inducible gene expression and gene modification in transgenic mice. $J$ Am Soc Nephrol 11 Suppl 16, S95-S100.

Kablar, B., Vignali, R., Menotti, L., Pannese, M., Andreazzoli, M., Polo, C., Giribaldi, M. G., Boncinelli, E. and Barsacchi, G. (1996). Xotx genes in the developing brain of Xenopus laevis. Mech Dev 55, 145-58. 
Keller, R. (1991). Early embryonic development of Xenopus laevis. Methods Cell Biol 36, 61-113.

Keller, R. (2002). Shaping the vertebrate body plan by polarized embryonic cell movements. Science 298, 1950-4.

Kumasaka, M., Sato, H., Sato, S., Yajima, I. and Yamamoto, H. (2004). Isolation and developmental expression of Mitf in Xenopus laevis. Dev Dyn 230, 107-13.

Kwak, I., Tsai, S. Y. and DeMayo, F. J. (2004). Genetically engineered mouse models for lung cancer. Annu Rev Physiol 66, 647-63.

Lee, Y. S. and Carthew, R. W. (2003). Making a better RNAi vector for Drosophila: use of intron spacers. Methods 30, 322-9.

Li, X., Wikramanayake, A. H. and Klein, W. H. (1999). Requirement of SpOtx in cell fate decisions in the sea urchin embryo and possible role as a mediator of beta-catenin signaling. Developmental Biology 212, 425-39.

Li, Z., Joseph, N. M. and Easter, S. S., Jr. (2000). The morphogenesis of the zebrafish eye, including a fate map of the optic vesicle. Dev Dyn 218, 175-88.

Link, C. D. (2001). Transgenic invertebrate models of age-associated neurodegenerative diseases. Mech Ageing Dev 122, 1639-49.

Livesey, F. J. and Cepko, C. L. (2001). Vertebrate neural cell-fate determination: lessons from the retina. Nature Reviews Neuroscience 2, 109-18.

Lovicu, F. J., de Iongh, R. U. and McAvoy, J. W. (1997). Expression of FGF-1 and FGF-2 mRNA during lens morphogenesis, differentiation and growth. Curr Eye Res 16, 222-30. 
Lovicu, F. J. and McAvoy, J. W. (2001). FGF-induced lens cell proliferation and differentiation is dependent on MAPK (ERK1/2) signalling. Development 128, 5075-84. Martinez-Morales, J. R., Signore, M., Acampora, D., Simeone, A. and Bovolenta, P. (2001). Otx genes are required for tissue specification in the developing eye.

Development 128, 2019-30.

Matsuo, I., Kuratani, S., Kimura, C., Takeda, N. and Aizawa, S. (1995). Mouse Otx2 functions in the formation and patterning of rostral head. Genes Dev 9, 2646-58.

McAvoy, J. W., Chamberlain, C. G., de Iongh, R. U., Hales, A. M. and Lovicu, F. J. (1999). Lens development. Eye 13 ( Pt 3b), 425-37.

Mochii, M., Mazaki, Y., Mizuno, N., Hayashi, H. and Eguchi, G. (1998). Role of Mitf in differentiation and transdifferentiation of chicken pigmented epithelial cell. Developmental Biology 193, 47-62.

Montalta-He, H., Leemans, R., Loop, T., Strahm, M., Certa, U., Primig, M., Acampora, D., Simeone, A. and Reichert, H. (2002). Evolutionary conservation of otd/Otx2 transcription factor action: a genome-wide microarray analysis in Drosophila. Genome Biol 3, RESEARCH0015.

Montgomery, M. K. (2004). RNA interference: historical overview and significance. Methods Mol Biol 265, 3-21.

Morgan, R., Hooiveld, M. H., Pannese, M., Dati, G., Broders, F., Delarue, M., Thiery, J. P., Boncinelli, E. and Durston, A. J. (1999). Calponin modulates the exclusion of Otx-expressing cells from convergence extension movements. Nat Cell Biol 1, 404-8. 
Nieuwkoop, P. D. and Faber, J. (1967). Normal table of Xenopus laevis. Amsterdam: North Holland Publishing.

Nishida, A., Furukawa, A., Koike, C., Tano, Y., Aizawa, S., Matsuo, I. and Furukawa, T. (2003). Otx2 homeobox gene controls retinal photoreceptor cell fate and pineal gland development. Nat Neurosci 6, 1255-63.

Nornes, H. O., Dressler, G. R., Knapik, E. W., Deutsch, U. and Gruss, P. (1990). Spatially and temporally restricted expression of Pax 2 during murine neurogenesis. Development 109, 797-809.

Osterwalder, T., Yoon, K. S., White, B. H. and Keshishian, H. (2001). A conditional tissue-specific transgene expression system using inducible GAL4. Proceedings of the National Academy of Sciences of the United States of America 98, 12596-601.

Perea-Gomez, A., Lawson, K. A., Rhinn, M., Zakin, L., Brulet, P., Mazan, S. and Ang, S. L. (2001). Otx2 is required for visceral endoderm movement and for the restriction of posterior signals in the epiblast of the mouse embryo. Development 128, 753-65.

Perron, M., Kanekar, S., Vetter, M. L. and Harris, W. A. (1998). The genetic sequence of retinal development in the ciliary margin of the Xenopus eye. Developmental Biology 199, 185-200.

Raymond, S. M. and Jackson, I. J. (1995). The retinal pigmented epithelium is required for development and maintenance of the mouse neural retina. Curr Biol 5, 1286-95.

Rhinn, M., Dierich, A., Le Meur, M. and Ang, S. (1999). Cell autonomous and noncell autonomous functions of Otx2 in patterning the rostral brain. Development 126, 4295-304. 
Roman, G., Endo, K., Zong, L. and Davis, R. L. (2001). P[Switch], a system for spatial and temporal control of gene expression in Drosophila melanogaster. Proceedings of the National Academy of Sciences of the United States of America 98, 12602-7.

Rorth, P. (1996). A modular misexpression screen in Drosophila detecting tissuespecific phenotypes. Proceedings of the National Academy of Sciences of the United States of America 93, 12418-22.

Rorth, P., Szabo, K., Bailey, A., Laverty, T., Rehm, J., Rubin, G. M., Weigmann, K., Milan, M., Benes, V., Ansorge, W. et al. (1998). Systematic gain-of-function genetics in Drosophila. Development 125, 1049-57.

Ryffel, G. U., Werdien, D., Turan, G., Gerhards, A., Goosses, S. and Senkel, S. (2003). Tagging muscle cell lineages in development and tail regeneration using Cre recombinase in transgenic Xenopus. Nucleic Acids Res 31, e44.

Shook, D. R., Majer, C. and Keller, R. (2004). Pattern and morphogenesis of presumptive superficial mesoderm in two closely related species, Xenopus laevis and Xenopus tropicalis. Developmental Biology 270, 163-85.

Simeone, A., Acampora, D., Mallamaci, A., Stornaiuolo, A., MR, D. A., Nigro, V. and Boncinelli, E. (1993). A vertebrate gene related to orthodenticle contains a homeodomain of the bicoid class and demarcates anterior neuroectoderm in the gastrulating mouse embryo. EMBO Journal 12, 2735-47.

Svoboda, K. K. and O'Shea, K. S. (1987). An analysis of cell shape and the neuroepithelial basal lamina during optic vesicle formation in the mouse embryo. Development 100, 185-200. 
Takeichi, M. (1988). The cadherins: cell-cell adhesion molecules controlling animal morphogenesis. Development 102, 639-55.

Tijsterman, M. and Plasterk, R. H. (2004). Dicers at RISC; the mechanism of RNAi. Cell 117, 1-3.

Torres, M., Gomez-Pardo, E. and Gruss, P. (1996). Pax2 contributes to inner ear patterning and optic nerve trajectory. Development 122, 3381-91.

Viczian, A. S., Vignali, R., Zuber, M. E., Barsacchi, G. and Harris, W. A. (2003). XOtx $5 \mathrm{~b}$ and XOtx 2 regulate photoreceptor and bipolar fates in the Xenopus retina. Development 130, 1281-94.

Xu, L., Overbeek, P. A. and Reneker, L. W. (2002). Systematic analysis of E-, N- and P-cadherin expression in mouse eye development. Exp Eye Res 74, 753-60.

Zakin, L., Reversade, B., Virlon, B., Rusniok, C., Glaser, P., Elalouf, J. M. and Brulet, P. (2000). Gene expression profiles in normal and Otx2-/- early gastrulating mouse embryos. Proceedings of the National Academy of Sciences of the United States of America 97, 14388-93.

Zygar, C. A., Cook, T. L. and Grainger, R. M., Jr. (1998). Gene activation during early stages of lens induction in Xenopus. Development 125, 3509-19. 
Figure 4-1. A schematic comparison of eye morphogenesis in wild-type and Otx2-EnRinduced mutant embryos during the transition from the optic vesicle to the optic cup. Modified after Holt, 1980. Cell movements (arrows) involved in the progressive phases of the optic cup formation are represented in transverse sections of wild-type (A-D) and Otx2-EnR-expresssing (E-H) embryos at indicated developmental stages. (A, E) Optic vesicle stage. In wild-type embryo, there is a dorsal elongation (arrow) of the vesicle, leading to the formation of the thin layer of the future RPE. (B, F) Invaginating eye vesicle stage. In the wild-type, the presumptive neural retina is moving inward (open arrow), resulting in the apposition of two retinal layers. The optic fissure formation begins at the distal margin (arrowhead) of the ventral side of the optic vesicle $(C, G)$ Newly invaginated double-layered eye cup. Arrows in C indicate downward movement of the retinal cells for the completion of ventral retina formation. (D, H) Mature optic cup. In the wild-type, the ventral half of the retina is now as large as the dorsal half. Note that in Otx2-EnR-expresssing embryos, morphogenetic cell movements shown in the wild-type embryo are severely affected throughout the different steps of eye formation (see the text for details). The fates of territories within the retina are labeled with different colors as indicated. Le, lens; nt, neural tube; os, optic stalk; ov, optic vesicle. 


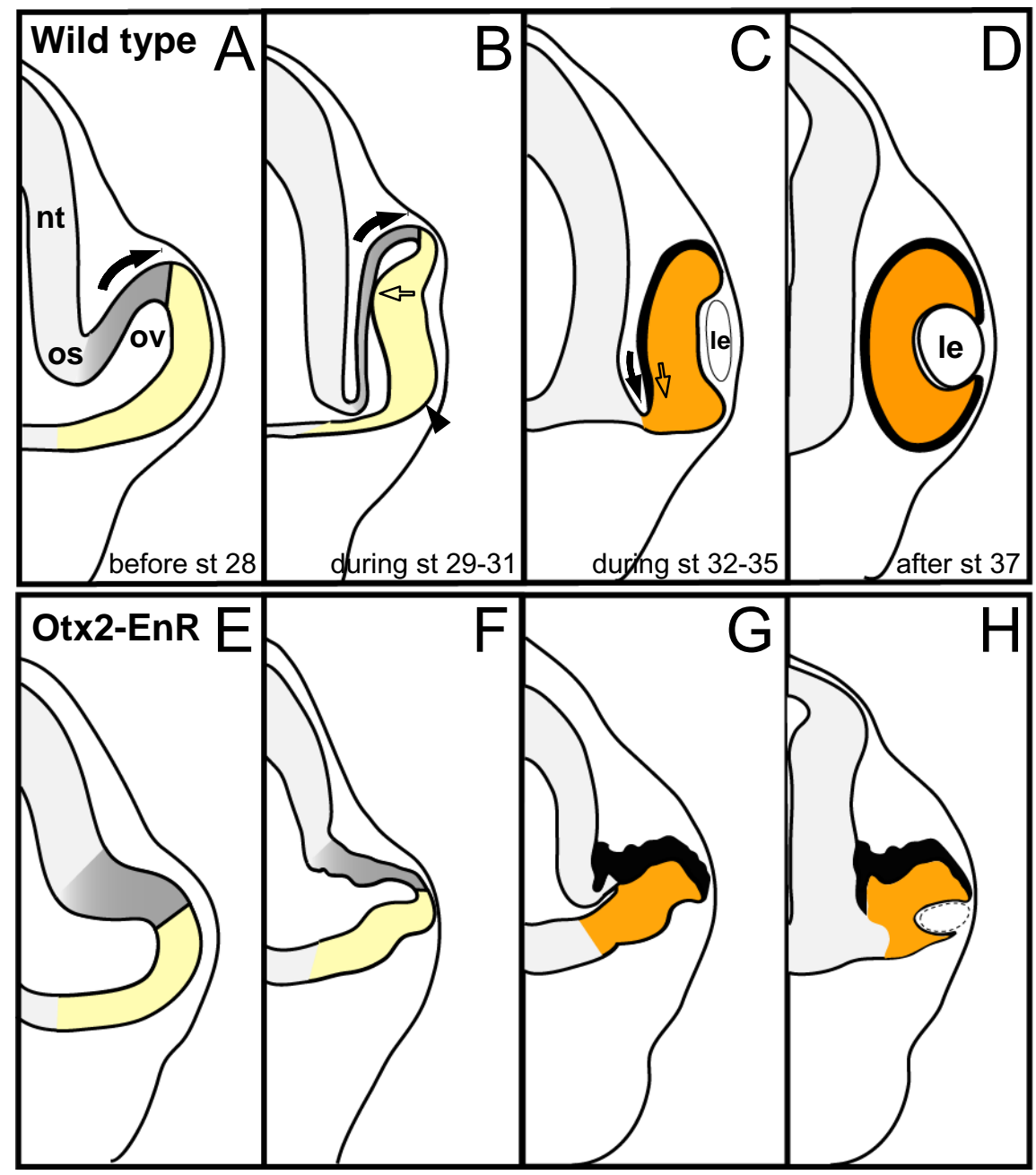

Fated for pigmented epithelium

Fated for neural retina

differentiating pigmented epithelium

differentiating neural retina 